\title{
A Phenomenological Photovoice Exploration of Female Exercisers' Experiences of their Body in Fitness Center Environments
}

\author{
Katherine E. Fairhurst \\ West Virginia University, kefairhurst@mix.wvu.edu
}

Follow this and additional works at: https://researchrepository.wvu.edu/etd

Part of the Health Psychology Commons, Public Health Education and Promotion Commons, Sports

Studies Commons, and the Women's Health Commons

\section{Recommended Citation}

Fairhurst, Katherine E., "A Phenomenological Photovoice Exploration of Female Exercisers' Experiences of their Body in Fitness Center Environments" (2020). Graduate Theses, Dissertations, and Problem Reports. 7546.

https://researchrepository.wvu.edu/etd/7546

This Dissertation is protected by copyright and/or related rights. It has been brought to you by the The Research Repository @ WVU with permission from the rights-holder(s). You are free to use this Dissertation in any way that is permitted by the copyright and related rights legislation that applies to your use. For other uses you must obtain permission from the rights-holder(s) directly, unless additional rights are indicated by a Creative Commons license in the record and/ or on the work itself. This Dissertation has been accepted for inclusion in WVU Graduate Theses, Dissertations, and Problem Reports collection by an authorized administrator of The Research Repository @ WVU.

For more information, please contact researchrepository@mail.wvu.edu. 
A Phenomenological Photovoice Exploration of Female Exercisers' Experiences of their Body in Fitness Center Environments

\author{
Katherine E. Fairhurst \\ Dissertation submitted \\ to the College of Physical Activity and Sport Sciences \\ at West Virginia University \\ in partial fulfillment of the requirements for the degree of \\ Doctorate of Philosophy in Kinesiology \\ Sport, Exercise, and Performance Psychology \\ Dana Voelker, Ph.D., Chair \\ Sam Zizzi, Ed.D. \\ Monica Leppma, Ph.D. \\ Christy Greenleaf, Ph.D., University of Wisconsin-Milwaukee \\ Department of Sport Sciences \\ Morgantown, WV \\ 2020
}

Keywords: Exercise Environment, Physical Activity, Embodiment, Body Image, Qualitative

Copyright 2020 Katherine Fairhurst 


\begin{abstract}
A Phenomenological Photovoice Exploration of Female Exercisers' Experiences of their Body in
\end{abstract}

Fitness Center Environments

\author{
Katherine E. Fairhurst
}

For many women, the relationship with their body and exercise is complex. Exercise can have positive effects on body image, however, not all women appear to benefit positively from all types of exercise. To date, body image research has focused on exercise as an activity and less so on the context in which exercise is performed. Women frequently exercise in fitness centers as young adults which, unfortunately, is associated with body dissatisfaction. Using an Interpretative Phenomenological Analysis (IPA) approach with Photovoice methodology, we explored young adult women's lived body experiences while exercising in fitness centers. A purposive sample of 11 women $\left(M_{\mathrm{age}}=21.9\right.$ years $)$ completed a two-phase study: (1) a two-week photography period and (2) a 60-90-minute, photo-elicited interview via Skype ${ }^{\mathrm{TM}}$. Three identified themes pertained to the sociocultural fitness setting, participants' fitness experiences, and how participants navigated their 'place' while exercising in fitness centers. Participants experienced a segregated fitness center environment driven by gender, the absence of female representation on machines, and "no place for women" in weightlifting areas. Interestingly, these negative experiences were buffered by self-compassion-based textual messages on walls and mirrors and dress codes which encouraged a harmonious relationship with their body while exercising. In light of these experiences, participants shared negotiating strategies (e.g., seeking private sub-spaces, challenging gender norms). This study elucidates the complexity of body experiences for young adult women in fitness settings and informs the development of exercise spaces that empower women to build a healthy relationship with their body through exercise. 


\section{Acknowledgements}

I would like to thank my family, friends, and mentors who have helped me through every hill and valley of this process - my gratitude for your support is endless. I thank Dr. Dana Voelker for challenging my insecurities, reaffirming my capabilities when my imposter syndrome reared its ugly head, and her constant reminders to take charge of my process. I am grateful for her unwavering standard of excellence and I could not be more appreciative of her guidance. I thank my committee members, Dr. Sam Zizzi who helped me keep healthy perspective and challenged me in my approach to the research, Dr. Monica Leppma who reminded me of the importance of self-compassion and self-acceptance, not only in the research but with myself, and Dr. Christy Greenleaf, who's early enthusiasm and support for my project gave me the confidence to jump all in. Thank you to Karly Casanave, a fellow graduate student, for her commitment and perspective - from the first time she expressed interest in contributing to this project to the final draft, the quality of the research has been elevated ten-fold because of her contribution. I would also like to thank Melinda Gallagher and Candice Brown, two research assistants whose skills far surpassed that of undergraduates - I'm grateful for your passion, commitment, and hunger to learn. Thank you to my colleagues and peers who read drafts, provided feedback, gave pep talks, and gave me the space to feel the full range of emotions that comes with the dissertation process; thank you Carra Johnson, Sofia Espana Perez, Matt Gonzalez, Adam Hansell, Dr. Robert Hilliard, Dr. Erika Van Dyke, Seth Swary, Bill Way, Dr. Zenzi Huysmans, Tommy Minkler, and Blake Costalupas.

As a final thank you, I thank my family. My parents, Barry and Lise, they are the foundation of who I am as a person, woman, helper, and giver. The Garretts, Gary and Melissa, who stepped in and adopted me into their family, took care of me and loved me when my family was far away. Shane, who has been by my side through it all. He was a source of unconditional love and late-night motivational speeches. He believed in me more than I did and made me laugh Every. Single. Day. 
Table of Contents

A Phenomenological Photovoice Exploration of Female Exercisers' Experiences of their Body in

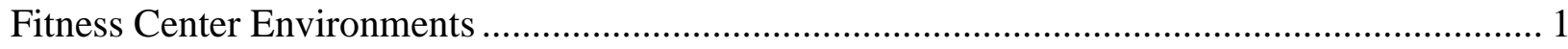

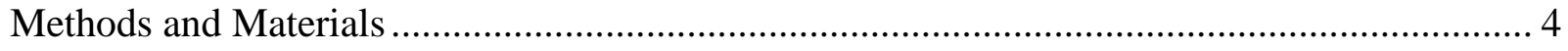

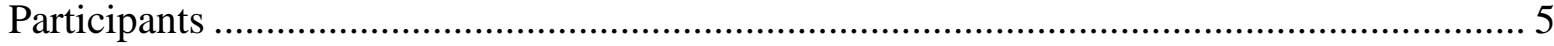

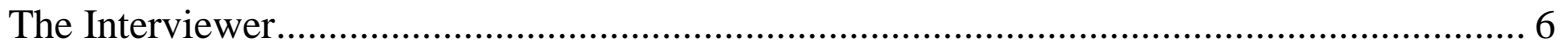

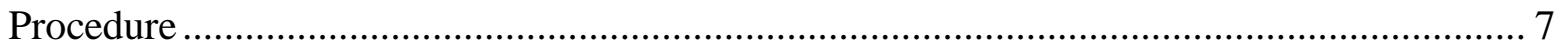

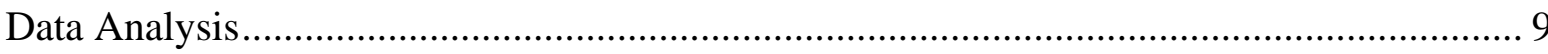

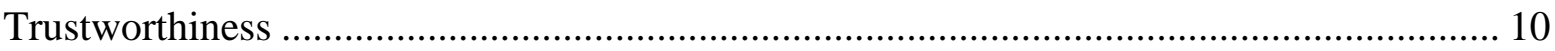

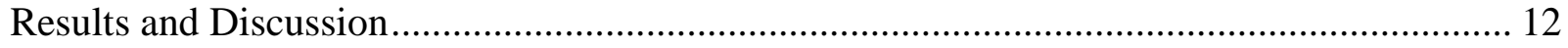

The Fitness Setting through a Sociocultural Lens ....................................................... 12

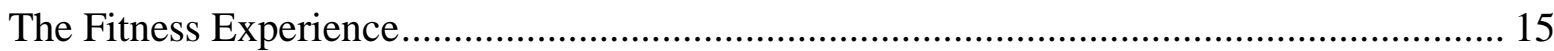

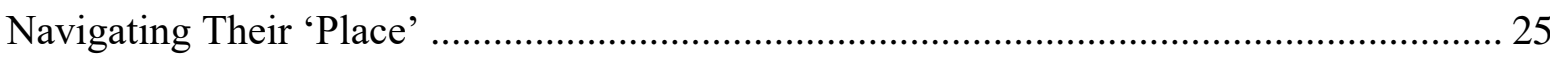

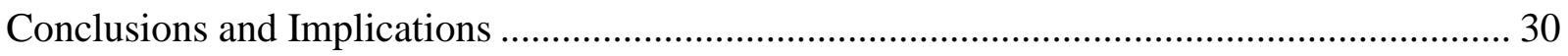

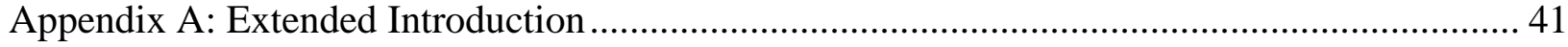

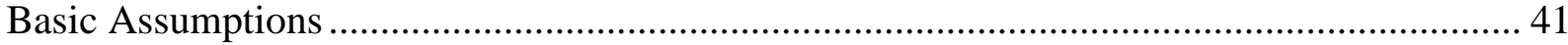

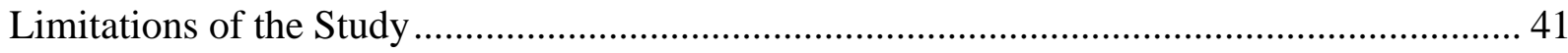

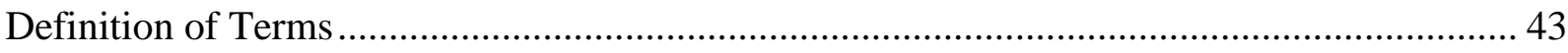

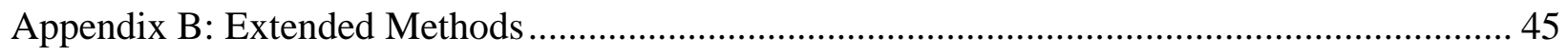

Interpretative Phenomenological Study Design .......................................................... 45

Phenomenology as a theoretical lens ..................................................................... 45

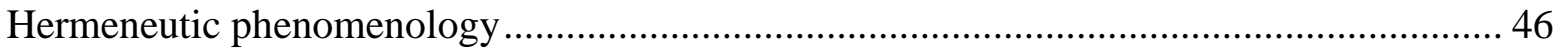

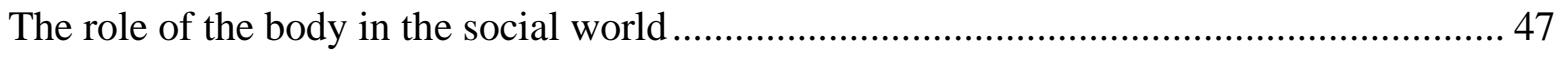

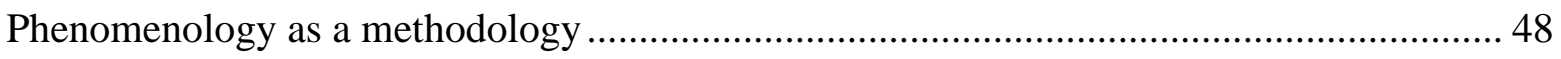

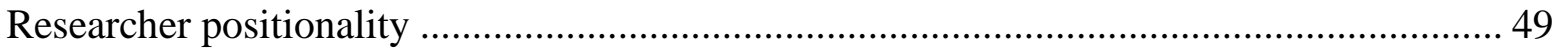

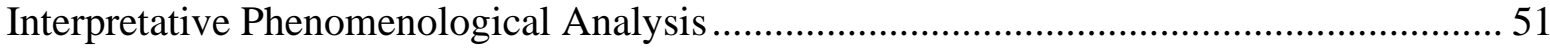

PhotoVoice approach within phenomenology ….............................................................. 52

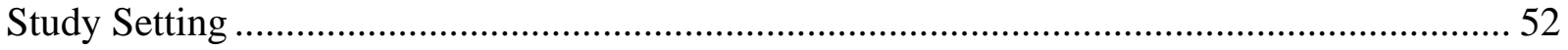

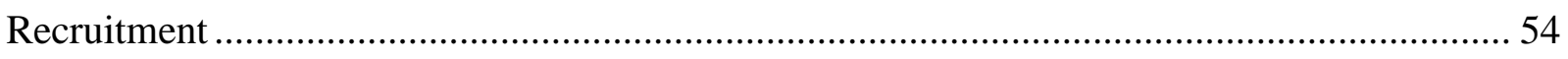

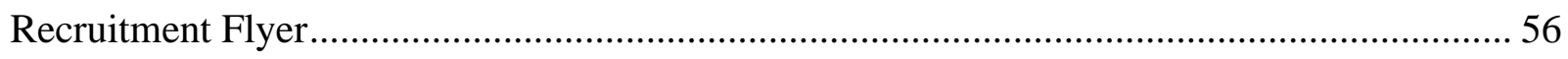

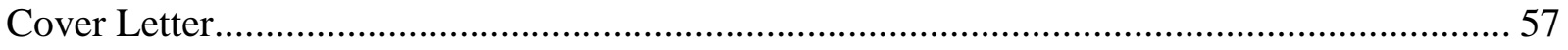

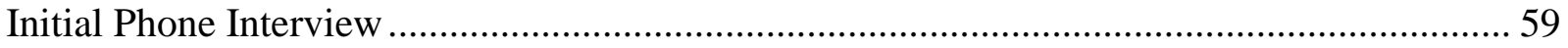

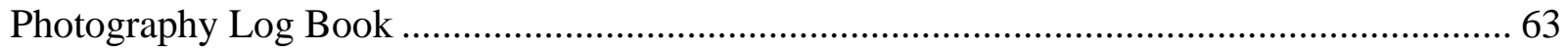


Photo Retrieval Process........................................................................................................ 64

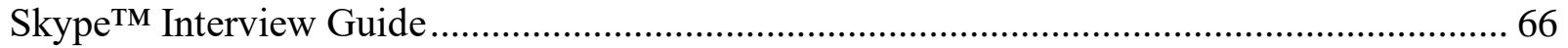

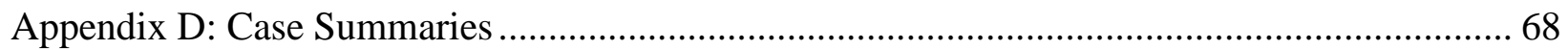

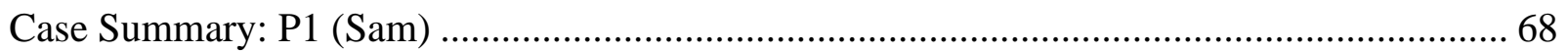

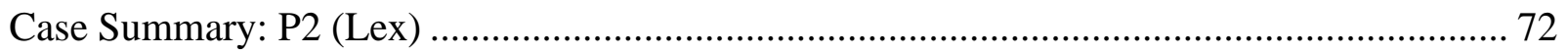

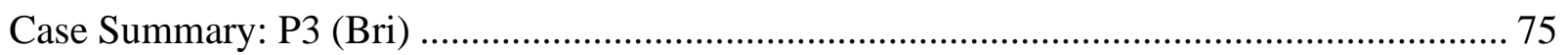

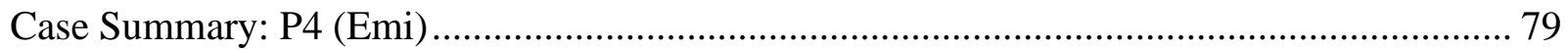

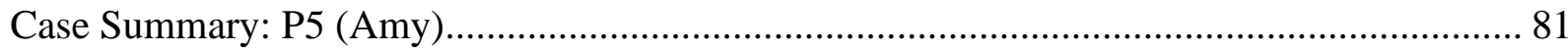

Case Summary: P6 (Ali) ............................................................................................. 85

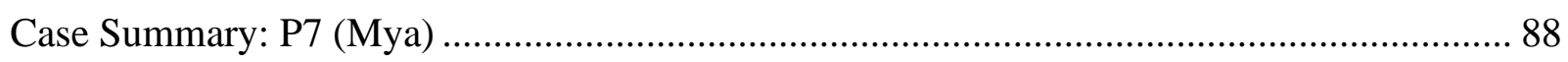

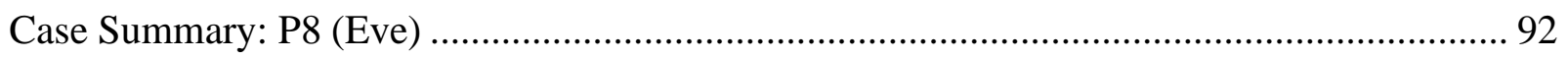

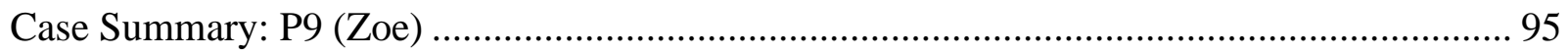

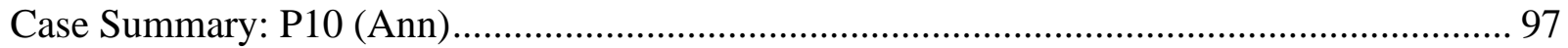

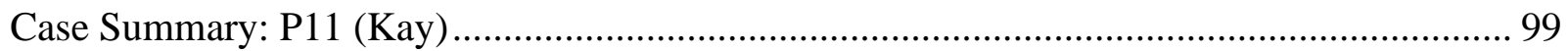

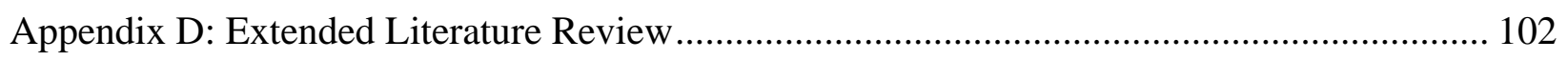

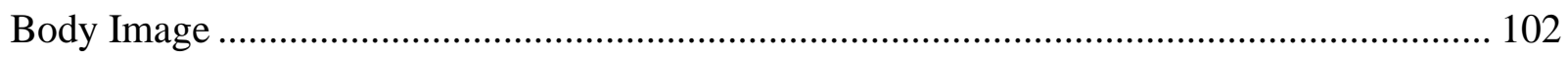

Body Image and Sociocultural Pressures .................................................................. 104

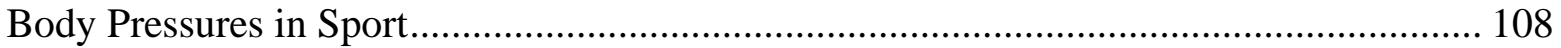

Body Pressures in Exercise Contexts ......................................................................... 117

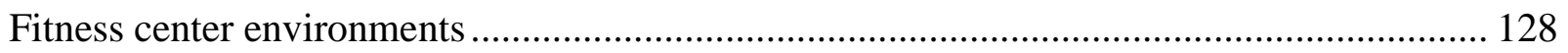

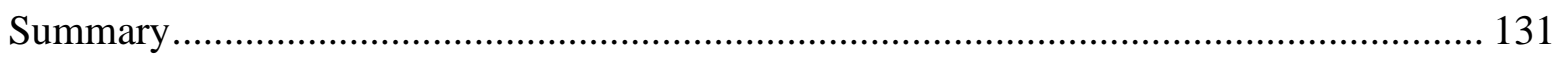




\section{A Phenomenological Photovoice Exploration of Female Exercisers' Experiences of their Body in Fitness Center Environments}

Western cultures are preoccupied with the gendered body (i.e., masculinity versus femininity) and emphasize appearance as a cultural value linked with identity and self-worth (see Bordo, 2004). Since the 1970 s, failure to obtain the socially-constructed body ideal has led to an increased prevalence of body dissatisfaction, especially among women (Neighbors \& Sobal, 2007). Women often attempt to change their body to achieve the feminine standard of thinness and beauty and, although most women will never achieve the cultural body ideal, many relentlessly work toward it through unhealthy diets and exercise (Voelker \& Reel, 2015).

In general, exercise has well-established physical benefits (e.g., Biddle \& Mutrie, 2007), and enhances a range of psychological factors, including body image (Campbell \& Hausenblas, 2009). Body image is a complex, multidimensional, psychological experience of embodiment, encompassing body-related self-perceptions, thoughts, feelings, and behaviors (Cash, 2004). Several meta-analyses have explored the effects of exercise on individuals who report body concerns. For example, Reel and colleagues (2007) found that exercise positively affected body image for both men and women, with anaerobic exercise (i.e., weight training) generating a stronger effect than aerobic exercises (i.e., jogging). Similarly, Campbell and Hausenblas (2009) found a significant effect for exercise on body image with no significant differences across several moderating factors, including exercise duration or length of intervention. Although research supports the positive effects of exercise on body image, much of this research focused primarily on exercise as an activity without accounting for the influence of context, or the sociocultural setting, in which exercise is performed. Therefore, to fully understand women's body image, particularly in relation to exercise, researchers must conceptualize these phenomena 
as environmentally-influenced experiences rooted within a rich sociocultural and historical context (see Cash, 2012).

The evolution of the fitness industry developed from the popularity of bodybuilding in the 1970's (Andreasson \& Johansson, 2014) and the social movement brought on by men who stayed home from the Vietnam war (Stern, 2008). Accordingly, fitness centers were established as places that valued the physical dominance of men (Klein, 1993), and prioritized strength training. Through the 1980's, the feminist movement and the rise of women's sports (Stern, 2008) encouraged women to engage in exercise for diverse, complex, and often paradoxical reasons. For example, feminist ideology deemed it necessary for women to display physical strength and independence to be perceived as socially empowered (Gottleib, 1981, as seen in Stern, 2008). Simultaneously, the body ideal expectations for women reinforced femininity, sexual attractiveness, and thinness-informed beauty (Silverstein, Peterson, \& Perdue, 1986), as reflected in society's message: '... if you only ate better and worked out more, you would be prettier and more successful in life and, especially, with men' (Stern, 2008, p. 9). In turn, aerobics classes and cardiovascular workouts were recommended as the preferred method of exercise for women due to their emphasis on weight loss and toning (Stern, 2008). The popularity of Jane Fonda's 1980's aerobics videos reinforced gender norms in fitness centers, solidifying the expectation that women should use cardiovascular machines and aerobics classes while 'real' men engaged in strength training (Dworkin, 2003).

Originally, the bodybuilding subculture was exclusively a male domain, however, the feminist movement and a shift in the ideal body has since attracted more women into the strength training areas of fitness centers (Andreasson \& Johansson, 2014). Communicated through 'fitspiration' messaging, the contemporary 'fit ideal' for women depicts increased muscularity, 
characterized by toned, lean physiques and extremely low body fat (Boepple, Ata, Rum, \& Thompson, 2016; Parviainen \& Aromaa, 2017). Young adult women, approximately one third of whom exercise in fitness centers (Slater \& Tiggemann, 2006), are a targeted 'fitspiration' audience (see Robinson, Prichard, Nikolaidis, Drummond, Drummond, \& Tiggemann, 2017). Fitness centers can offer young adult women an important context to develop a healthy and empowered relationship with exercise that supports a positive body image, and generally, fitness centers intend to create a positive exercise climate. Unfortunately, fitness centers are often characterized by numerous features (e.g., full-length mirrors, posters which idealize the female body) that communicate and reinforce unique appearance pressures for young adult women (Prichard \& Tiggemann, 2005). Internalizing these appearance pressures is associated with selfobjectification or the habitual monitoring of one's outward appearance (Fredrickson \& Roberts, 1997), which can contribute to body image concerns and unhealthy weight management practices (e.g., over-exercising, disordered eating; Chang, Pan, \& Shu, 2018).

Thus far, research on body image demonstrates that fitness centers have a formative impact on young adult women's exercise experiences (e.g., Prichard \& Tiggemann, 2005; 2012), particularly during a critical developmental period where self-consciousness is heightened (Noll \& Fredrickson, 1998) and fitness center participation is high (Slater \& Tiggemann, 2006). However, few studies have examined socioculturally-informed body experiences specifically within the context of fitness centers. The developmental theory of embodiment (DTE; Piran, 2017) outlines pathways through which women experience their body and presents a rich framework for exploring this phenomenon. DTE encompasses the interactions between women and their social environments and their embodied experiences in those environments. Uniquely, DTE not only addresses negative experiences and pressures but also honors facilitative, 
empowering experiences and social factors, promoting this theory as a holistic framework for exploring young adult women's complex exercise experiences. Therefore, the purpose of this study was to explore young adult women's lived experiences of their body while exercising in fitness centers. The following research questions guided the study: (a) how do young adult women exercisers experience their body (e.g., weight, shape, size, appearance) while exercising in fitness centers? and (b) what specific elements of their fitness center environments do they perceive to positively, and negatively, influence how they experience their body (e.g., weight, shape, size, appearance) within the fitness center setting? Ultimately, this study provides insight on how exercise professionals can advocate for fitness environments that cultivate a healthy body culture and empower young adult women to engage in healthy relationships with themselves and exercise.

\section{Methods and Materials}

An Interpretative Phenomenological Analysis (IPA) approach (Pietkiewicz \& Smith, 2014), with a Photovoice method (Wang \& Burris, 1994; 1997), was used in this study. In accordance with the hermeneutic phenomenology tradition, the first author was interested in historicality (Laverty, 2003); that is, how a person's lived experience, and understanding of the world, is enmeshed with their historical, cultural, and social contexts (Munhall, 1989). To examine a lived experience within this tradition, detailed descriptions of an individual's background, pre-understandings, co-constitution, and interpretation provide context for how a person makes meaning of their experiences (Benner \& Wrubel, 1989). Background is defined as a personal history - an inextricable part of a person that culture imparts on them from birth - and is used to explain how people can experience similar phenomena in different ways (Kerry \& Armour, 2000). Heidegger (1927/1962) used the term pre-understandings to describe the 
structure of a culture as it exists, prior to a person's interaction with, and interpretation of, that culture. Co-constitution relates to pre-understandings as it highlights the link between a person and the world such that 'we are constructed by the world in which we live, and, at the same time, we perceive the world from our own experience and background' (Kerry \& Armour, 2000, p. 6). Finally, Heidegger (1927/1962 as cited in Taylor, 1985) argued that every social interaction involves interpretation - humans are self-interpretative beings, constantly redefining the self through acts of interpretation of the culture in which they exist. Consistent with the idiographic approach of IPA (Pietkiewicz \& Smith, 2014), this study was based on photo-elicited, semistructured interviews with a small sample of young adult women recruited through purposive sampling. To capture the real time, lived experience of women's body experiences in fitness centers, this study used a Photovoice method (Wang, 1999). The Photovoice approach used represents a blended process of photography and narratives to explore social issues (Wang \& Burris, 1994; 1997). Photovoice allowed the first author to richly describe and visualize participants' perceptions of their everyday realities. This was accomplished by allowing participants to (a) document, with personal expression, factors in fitness centers that were integral in their interpretation of their body experiences during exercise and (b) describe, in depth, their lived experience of interacting with those factors through photos captured.

\section{Participants}

Participants were 11 young adult women aged $18-25$ years $\left(M_{\mathrm{age}}=21.8\right.$ years $)$ who identified as white $(n=10)$ and white/Native American $(n=1)$. As active exercisers, they attended their current fitness center for an average of 1.74 years, exercising 4-7 days per week $(M=4.55$ days/week) at college campus recreation centers $(n=3)$, commercial chains $(n=3)$, independent studios $(n=2)$, CrossFit $(n=1)$, and employee wellness facilities $(n=2)$ across four 
states (see Table 1). All the participants were former competitive athletes who transitioned into exercising in fitness centers after sport. Two women reported continued participation in competitive sport during the study.

Table 1

Demographic Characteristics of Participants Based on Inclusion Criteria

\begin{tabular}{lllll}
\hline Pseudonym & Age & Occupation & Type of Fitness Center & $\begin{array}{l}\text { Days/week } \\
\text { at Fitness } \\
\text { Center }\end{array}$ \\
\hline Sam & 25 & Employed Full-Time & Commercial Chain & 3 \\
Lex & 23 & Graduate Student & College Recreation Center & 5 \\
Bri & 22 & Graduate Student & Independent Studio & 6 \\
Emi & 20 & Undergraduate Student & CrossFit Gym & 5 \\
Amy & 21 & Graduate Student & Commercial Chain & 4.5 \\
Ali & 24 & Employed Full- & Commercial Chain & 3 \\
& & Time/Graduate Student & & \\
Mya & 19 & Undergraduate Student & Independent Studio & 5.5 \\
Eve & 24 & Employed Full-Time & Employee Wellness Facility & 5 \\
Zoe & 18 & Undergraduate Student & College Recreation Center & 4.5 \\
Ann & 20 & Undergraduate Student & College Recreation Center & 4 \\
Kay & 24 & Employed Full-Time & Employee Wellness Facility & 4.5 \\
\hline
\end{tabular}

\section{The Interviewer}

Hermeneutic phenomenology aligns with the first author's relativist ontological position, (i.e., the interviewer); she acknowledges the existence of multiple realities which are interpreted through the co-construction of experience throughout the participant-researcher exchange (Ponterotto, 2005). The first author participated in elite aesthetic sport for 15 years followed by regular exercise in fitness centers, which was fueled by appearance-focused motives. The first author has a history of a negative relationship with her body and exercise, eating pathology, and exercise dependence. She has since engaged in an active self-growth journey of mindfulness and self-compassion, from which she developed a healthy, empowered identity as a woman in fitness. Additionally, the first author has post-secondary training in mental health counseling and 
is a Certified Personal Trainer with experience training young adult women. Throughout this study, the first author consistently examined her personal assumptions by practicing Le Vasseur's (2003) stance of persistent curiosity - simultaneously being mindful of her role in the interpretation of the phenomenon while being open to fresh and new perceptions that might occur. This stance of curiosity allowed rich data interpretation as it created a nonjudgmental and compassionate space for the first author and the participants to openly discuss potentially sensitive experiences.

\section{Procedure}

Upon Institutional Review Board approval, convenience and snowball sampling methods were used to identify potential participants. Flyers with eligibility criteria were posted to social media outlets and sent via email to contacts who may know interested participants (e.g., colleges, fitness centers, fitness professionals). Maximum variation sampling (Patton, 2001) was employed relative to type and culture of community-based fitness centers. Interested participants who contacted the first author engaged in a short screening call to confirm eligibility, answer questions, obtain informed consent, and schedule the study. Data collection occurred in two phases: (1) initial phone interview followed by a two-week photography period and (2) a 60-90minute Skype ${ }^{\mathrm{TM}}$ interview followed by member reflections. Each phase was piloted with two young adult female exercisers aged 23 and 25 years old to test the feasibility and clarity of the study protocol; the pilot study informed changes that clarified participant scheduling and photography instructions.

Phase 1. The first phase of data collection consisted of audio-recorded phone interviews, guided by a script, to build rapport and understand the participants' perceptions of physical and cultural aspects of the fitness culture and their individual fitness centers. The initial phone call 
was also used to provide instructions for taking photographs over a two-week period with a digital camera. Instructions were to (a) take pictures with the camera embedded in their cellular smartphone, (b) focus their photography toward the directive prompt that was provided, (c) document additional notes or insights not captured in the photographs using a 'log book' (Plunkett, Leipert, \& Ray, 2013), and (d) indicate their preference for phone or text reminders during the photography period. These interviews lasted between 17-29 minutes.

To complete the two-week photography task, participants were prompted with the 'photography mission' (Nykiforuk, Vallianatos, \& Nieuwendyk, 2011, p. 109) to 'take photographs of elements in your fitness center that are encouraging, and discouraging, to how you feel, think, and perceive your body while exercising'. Encouraging was defined as to 'inspire with courage, spirit, and hope' (Merriam-Webster, 2020), and discouraging was defined as to 'make less determined, hopeful, or confident' (Merriam-Webster, 2020). Minimal direction beyond this prompt was given, nor a minimum number of required photographs, to promote participant-driven exploration.

Phase 2. The second phase of data collection consisted of audio-recorded, photo-elicited, semi-structured interviews via Skype ${ }^{\mathrm{TM}}$ (i.e., 34-80 minutes in length) to capture participants' background, pre-understandings, co-constitution and interpretation of the phenomenon. To begin, the first author asked about the participants' personal histories in relation to exercise (e.g., 'What motivated you to begin exercising in a fitness center?'), relationship with exercise (e.g., 'How would you describe how you feel about exercise?'), perceptions of the fitness culture, and motivations for exercising in a fitness center. Second, questions guided participants in describing their photographs using the screen-sharing function of Skype ${ }^{\mathrm{TM}}$. To maintain phenomenological consistency, participants were given the freedom to select the most descriptive and salient 
photographs and were prompted to elaborate on the photographs using questions derived from the SHOWeD method, a photovoice approach to interviewing (i.e., 'What do you see here?', 'How does this relate to your life?', 'Why does this situation exist?'; Wang, 1999). Third, participants were asked to discuss ideas that may not have been captured through their photography (e.g., 'What other factors, not captured by photographs, are encouraging to your body image while exercising? Discouraging?'). Finally, participants were encouraged to share their general perceptions of women in fitness (e.g., 'Reflecting on this experience, what meaning does exercising in a fitness center give your body?'). Upon the completion of phase 2, each participant received a $\$ 25$ electronic gift card to Amazon.com.

\section{Data Analysis}

Throughout each interview, the first author made reflections on the interview experience including aspects of content, language use, context, and any noteworthy comments related to personal reflexivity (Pietkiewicz \& Smith, 2014). All interviews were transcribed verbatim while coding any identifying information with a pseudonym and photo-editing to protect participant confidentiality. Pietkiewicz and Smith's (2014) practical guide for IPA in qualitative research was followed to analyze the data. In general, IPA provides flexible guidelines which can be adapted to the researchers' unique research objectives and questions. Phase 1 interview data were used to describe the participants, understand their exercise history, and elucidate their perceptions of the sociocultural context of fitness. Phase 2 interview data were used to give depth, description, and interpretation to the participants' photographs. Member reflection data were used to reflect the first author's interpretation and thematic representation of the participants' experiences back to the participants. 
To analyze the data, the first author engaged in multiple readings and note making of the transcripts and listened to the audio recordings of each interview. Next, the analysis included transforming notes into emergent themes by working mostly from notes to formulate concise phrases that captured a higher level of abstraction while remaining rooted in each participant's account. The final stage of analysis consisted of seeking relationships and clustering themes where the previously formulated concise phrases were analyzed to seek relationships between phrases. These concise phrases were clustered into themes according to conceptual and interpretative similarities and subthemes were included to capture essential depth and nuance. .

\section{Trustworthiness}

In addition to prolonged engagement and triangulation from multiple data sources (e.g., log books, verbal reflection; Yardley, 2008), the first author developed written case summaries based on interview transcripts, photographs, and analyses to clarify main ideas, note initial interpretations, and identify follow-up questions for clarification and further participant reflection. These case summaries served as the basis for member reflections via phone with each participant and afforded an additional opportunity for the participant and first author to coparticipate in reflection of interpretation more broadly and the interview data specifically (Smith \& McGannon, 2018). All 11 participants were contacted for member reflections; nine responded and completed a phone call lasting 10 to 20 minutes. As the first author shared her interpretations through case descriptions, some of the participants provided additional insight after engaging in more reflection. The first author recorded participants' comments and insights to the case summaries.

Additionally, the third author acted as a 'critical friend' at multiple points during the analysis (Smith \& McGannon, 2018). The role of a critical friend is to encourage critical 
dialogue between researchers; a theoretical sounding board to reflect on alternative interpretations that might emerge during the analysis process (Smith \& McGannon, 2018). The critical friend role provided additional perspective and encouraged the first author's reflexive approach. Specifically, in reviewing the initial notes of each participant, the third author identified similarities between the raw data and the emergent themes, suggesting that the interpretation required increased rigor. The third author also highlighted similarities in data interpretations across clustering of the emergent themes early in the analysis process, which clarified and refined subsequent steps. Importantly, the third author consistently encouraged the first author's reflexivity process by asking challenging questions. For example, the first and third authors have a range of body-related experiences in fitness, allowing the third author to offer alternative perspectives for initial interpretations (e.g., Does the participant's hesitation to answer represent discomfort or reflection? What differentiates the fitness center areas objectively by gender? How? Why are women in this study so strongly driven to find comfort in their fitness center?). This reflective process allowed the first author to maintain perspective on her positioning with the data and maintain alignment with the phenomenon being explored.

Finally, the second author completed a series of 'abridged audits' conducted in accordance with recommendations from Smith, Flowers, and Larkin (2009). These 'abridged audits' consisted of the second author (faculty advisor) reviewing a single interview transcript annotated with initial notes and emergent themes. The auditor then critically reviewed the annotations for trustworthiness, transparency, and credibility including a logical step-by-step path from the text to formulation of themes (Smith, Flowers, \& Larkin, 2009). Four individual abridged audits were completed during the analysis. 


\section{Results and Discussion}

Through the Photovoice data, participants interpreted, and found meaning in, their body through a shared experience which described power and gender dynamics while exercising in this unique context. In the sections that follow, we elucidate each of the three over-arching themes, namely the sociocultural fitness setting, their fitness experiences, and how they navigated their sense of 'place' while exercising in fitness centers.

\section{The Fitness Setting through a Sociocultural Lens}

How the participants experienced their body while exercising was uniquely informed by their perceptions of the fitness culture. This theme, the fitness setting through a sociocultural lens, details the participants' pre-understandings of the context of fitness - the social, historical, and cultural landscape of fitness from both broader society and within their specific fitness centers. When asked to share their perceptions of the fitness culture broadly, all the participants described appearance expectations communicated by social media (e.g., Instagram). Participants specifically described an unrealistic fit ideal characterized by a muscular body with 'six pack abs' (Eve), 'cuts [sic] in her arms' (Amy), 'definition without bulking up' (Amy), and an 'hourglass figure - flat stomach' (Mya) that is also 'curvy, the way a woman should be' (Sam). In perpetuating this fit ideal, some participants explained that social media has 'become more about your appearance and less about the healthy lifestyle' (Lex). Bri shared:

Fitness influencers on Instagram for sure - I've seen a negative influence in that [sic] because they're spewing out all this information and they might not even be a certified personal trainer. They might be extremely fit and say, "you need to do this for your body" and people get discouraged if they don't look like that. It's kind of just this negative society. 
These notions are consistent with research examining the fit ideal across 'fitspiration' websites, which have been found to overemphasize the importance of appearance and provide controversial recommendations, such as over-exercising and restrictive dieting (Boepple, Ata, Rum, \& Thompson, 2016), to which participants in our study were acutely aware.

As part of the fit ideal, participants described expectations, communicated by athletic apparel companies, to wear tight, form-fitting, and revealing clothing while exercising. Eve explained: 'there's now clothing lines that are geared towards - like you [need to] have this specific body type to pull off, like just a sports bra. When was the last time you cared about what you look like when you're going to the gym?!' Ali similarly expressed: '... but I just want to wear my black leggings and a big sweatshirt. And then I feel like people are going to judge me...'. This fear of judgement is consistent with literature suggesting that being taken seriously in a fitness environment is associated with wearing 'purpose-made, gym-specific' clothing (Sassatelli, 1999, p. 231) and 'creates a fuzzy line between health and being perfect' for women (Fisher, Berbary, \& Misener, 2018, p. 486), even prior to entering the fitness center to exercise. In pursuit of the fit ideal in the 'right' clothes, participants described women's motivations to exercise as appearance-focused, as Zoe described: 'I don't think many girls have ever walked into a gym not because there was something that they wanted to fix'. Mya even noted that individuals will pursue the fit ideal with extreme, self-punishing workouts: 'People that don't classify themselves as fit, if they don't look the part, what they say is 'I'm $d y$ ing at the gym", or "killing myself at the gym". They have to hurt themselves to look like that, you know?' Research suggests that exposure to, and the pursuit of, the fit ideal is associated with a range of negative experiences. Observing 'fitspiration' content on social media, for example, has been shown to increase body dissatisfaction in young adult women (Robinson, Prichard, Nikolaidis, 
Drummond, Drummond, \& Tiggemann, 2017) while wearing fashionable, tight-fitting clothes has been shown to increase self-objectification and -surveillance (Prichard \& Tiggemann, 2005). Participants in the current study recognized that an appearance-focused fitness culture is misguided, such that the fit ideal for women is unrealistic and fails to embrace body diversity. In addition to strict body- and apparel-expectations from broader society, participants also described the presence of strong gender norms in their fitness centers, which were perceived negatively. Zoe noticed how her fitness center was separated by gender-stereotyped exercise modalities:

I don't even know if [the fitness center] says much about women. The main gym is for weightlifting and I don't really think the way that it's designed gives a place for women. Conveniently, cardio, which is what a lot of women do, is in a whole separate space and all of the classes are held in completely different areas of the gym. So, I think subconsciously and, hopefully not on purpose, it was designed in a way that put women elsewhere. My guess is that it was probably a - the person who probably did [sic] the layout of the gym was probably a white male.

Early literature suggested that fitness centers were historically masculine institutions that prioritized the physical dominance of men (Klein, 1993) and were designed in ways that reinforced gender segregation (Craig \& Liberti, 2007). For Ali, the fitness center was experienced in a gender-segregated way which ultimately influenced her early perceptions of where women belong in fitness:

...rec center had a lot of different rooms. One room would be cardio, one room would be the weight machines, and then another would be the regular weights. And the room with the regular weights, there was all the big-time weightlifter 
guys and stuff and no girls, so it wasn't really comfortable going in there, so I'd just stick with the cardio machines - I guess it's like specific areas for specific people.

These findings are consistent with evidence that fitness centers are built in ways that reinforce gender lines and segregation by structurally separating cardiovascular equipment intended for women from weight training machines intended for men (Dworkin, 2003). In sum, the participants' pre-understandings of the broad fitness culture included strict, appearance-focused body expectations, apparel standards for women, and fitness centers characterized by gender stereotypes and norms which, ultimately, were perceived as unwelcoming to women in fitness.

\section{The Fitness Experience}

Exercising within the context of fitness centers resulted in a complex experience for the participants. Through the Photovoice data, this theme, The Fitness Experience, elucidates common factors, discouraging and then encouraging, of the participants' fitness centers that influenced how they interacted with the setting and how they experienced their body while exercising there.

Discouraging factors. Participants perceived their fitness centers to be dominated by factors that were discouraging to their body experiences while exercising. Participants felt that fitness centers represented a space primed for self-evaluation. The presence of large mirrors, machines, as well as men gazing directly upon women in the weight area were identified as some of the common triggers. As such, the structural aspects of the exercise space itself represented a male-only, hegemonic masculine space which: 'just turns [women] away, where it's already like a men's club. So, you've already turned away like 90 percent of women' (Zoe). For example, Sam captured the masculine-dominated representation on weight machines at her fitness center: 
'on all weighted machines - it's the musculature of a man. It just goes back to being like "oh this is a man's workout". Like, fitness is for a man - kind of got a little bit of sexism in there.'

\section{Figure 1}

“This is a man's workout” by Sam

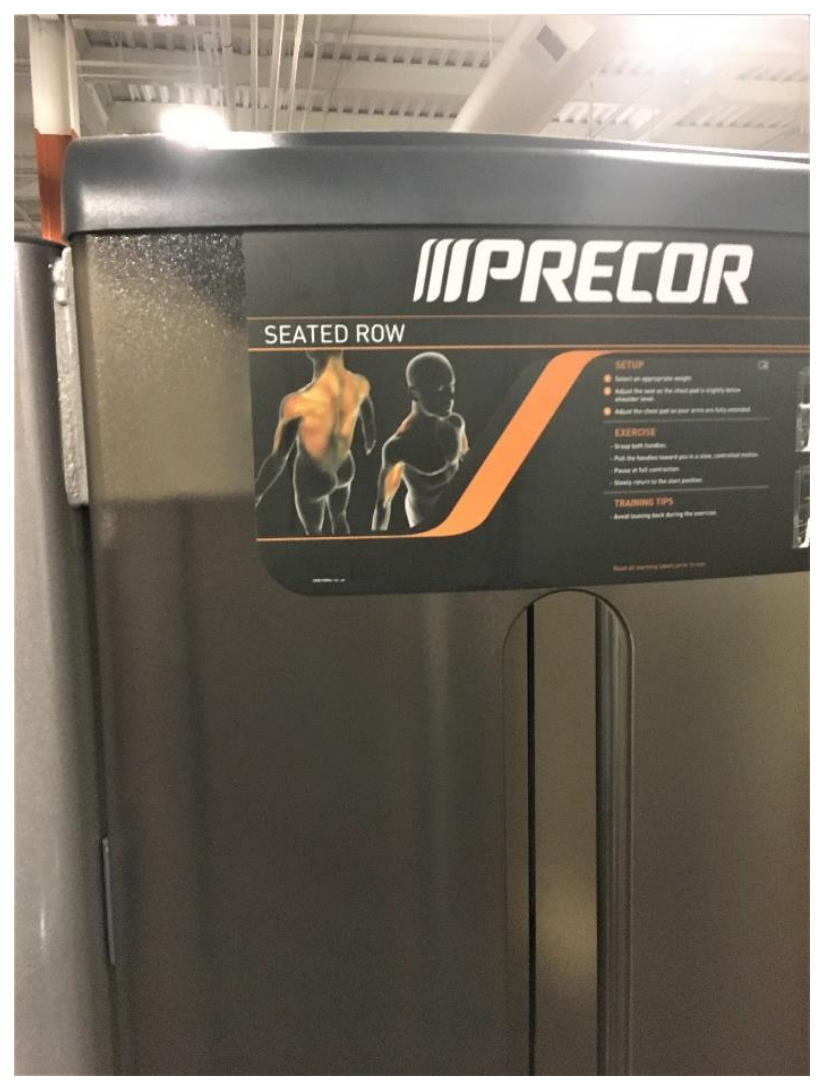

The presence of the male musculature on weight machines reinforced the message that weight training is for heteronormative men. Interestingly, the presence of this factor not only perpetuated hegemonic masculinity, but the absence of female representation was noted to increase the risk of self-doubt about belonging in the weight area. When asked to interpret what this meant to her about her exercising body, Sam declared: 'oh, if I do these exercises I'm going to look like a dude.' She elaborated on her personal frustrations at the gender expectations placed on women in fitness: 'I mean, [it's] like they're afraid of a strong woman.' In recent research, women have described this experience as being crowded out of spaces because of hegemonic 
masculinity and, as a result, felt the pressure to minimize their presence in male-dominated areas of the fitness center (Coen, Rosenberg, \& Davidson, 2018). In our study, not only was this message communicated by the predominance of male representation on weight machines, but also through the absence of actual equipment designed for women who lift weights. Traditional weightlifting bars are designed differently for men and women. For example, Rogue Fitness (2020) provides a 'Men's Ohio' bar and a 'Women's Bella' bar. The Bella bar is a bar length of 75.13 ", bar diameter of $25 \mathrm{~mm}$, and weight of $15 \mathrm{~kg}$, which is smaller than the men's Ohio bar which is 86.75 " in length, $28.5 \mathrm{~mm}$ in diameter, and $20 \mathrm{~kg}$ in weight. The differences are deliberate to accommodate purported differences in center of gravity and weight distribution and differences in hand and grip size between sexes (Rogue, 2020). Zoe, a member of a weightlifting club, photographed the barbells at her fitness center:

Figure 2

"They don't understand the difference" by Zoe

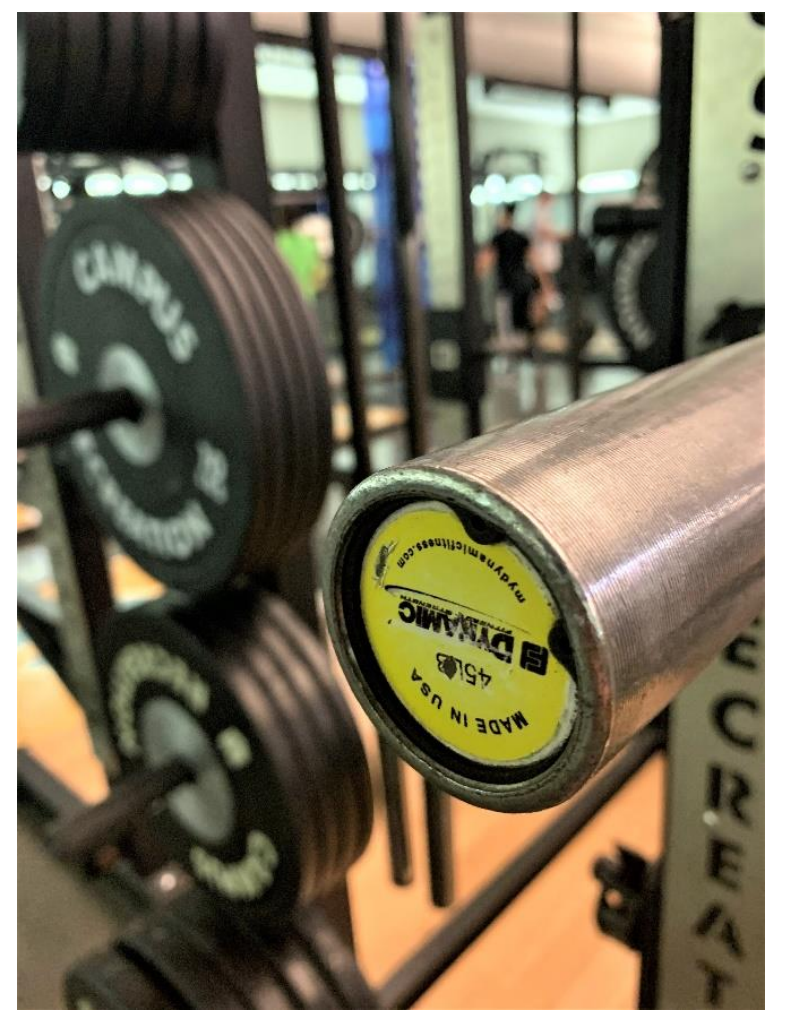


I don't know why they won't just buy women's bars. The idea that it's a men club is kind of pushed. Like, "this is a place for like guys to come and lift and get swole" and, you know, a girl at the rig sticks out like a sore thumb.

For many of the participants in our study, discomfort and fear of evaluation were salient experiences due to being highly visible as a woman in the male-dominated weight area. Many of the participants also described avoiding certain machines that triggered an uncomfortable, selfconscious experience. The assisted pull-up machine was photographed as a salient symbol for this threatening experience. Kay shared her vulnerability related to this machine - fear in her ability to perform the exercise and self-deprecating conclusions about her eating habits, weight, and body if unable to successfully complete a pull-up:

\section{Figure 3}

“Women can't do these” by Kay

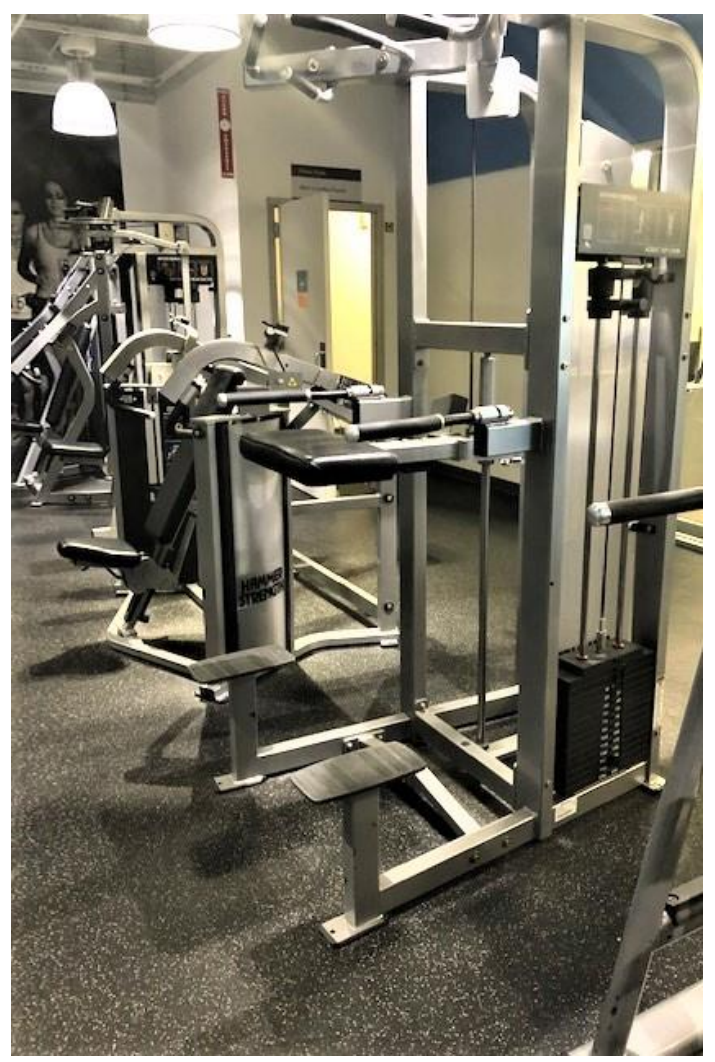


I don't like going on that machine just because I feel like it makes me super selfconscious. It's like, "oh well, you ate too much the past couple of days so that's why you can't lift yourself up and that's why you have to add more”. It actually becomes very, very negative when I have to do that. If you like can do it, it's like “oh my gosh, you can do a pull-up, that's amazing”. It kind of qualifies you as a fitness person in my mind.

These results elucidated that traditional fitness centers can have a restrictive influence on a woman's body experience based on the gender norms reinforced. Cockburn and Clarke (2002) found that adolescent girls similarly avoided experiences that either threatened the female identity or challenged one's ability to measure up to the socially-defined body ideal. For adult women in mixed-gendered gyms, avoidance of exercises may be due to risk, or fear, of not being able to perform the exercise in a confident way (Fisher, Berbary, \& Misener, 2018). Given traditional beliefs that women possess upper body strength inferior to men (Miller, MacDougall, Tarnopolsky, \& Sale, 1993), it is unsurprising that the women in our study avoided or felt uncomfortable using pullup machines. The perceived dominance of male representation on strength equipment, absence of representation and equipment designed for women, gender norms about strength, and equipment that posed a threat to their identity as a woman contributed to selfobjectification and self-consciousness about their belonging in a space defined by hegemonic masculinity.

Encouraging factors. Although the discussion was dominated by discouraging factors, the participants captured encouraging factors that were perceived to buffer negative experiences while exercising in their fitness centers. Most notably, a learning environment, apparel standards, the freedom to choose empowering exercises and machines, and self-acceptance-based messages 
in the fitness center encouraged positive body experiences while exercising. Emi shared that the combination of scaled workout options guided by a knowledgeable coach at her fitness center neutralized the fear of failing at difficult exercises. In contrast with past experiences at other fitness centers, Emi felt comfortable in her body in this environment because different levels of fitness experience were normalized:

\section{Figure 4}

“Being guided to success" by Emi

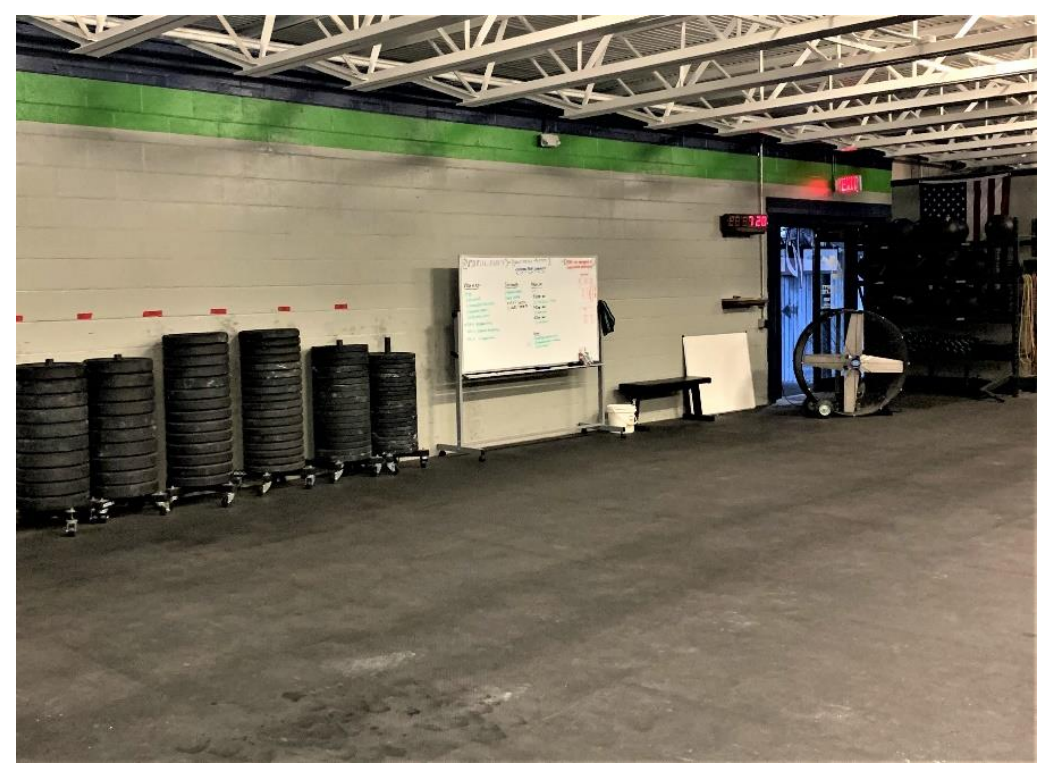

On the board, they'll put scaling options. "Here's the traditional weight, but also here are some other things that you could do if you're not comfortable doing that". I like the teaching slash learning aspect of it, that you're being guided to success, like you weren’t just - like I walked into [name of past fitness center] and just kind of did whatever I wanted to do. There's no guidance really.

For other participants, a positive body experience included the ability to create, choose, and scale exercises in their workout to meet their personal comfort levels with fitness. Amy captured this freedom to choose in a photograph of a long rack of different sized dumbbells: 'My favorite 
thing to see at the gym - one of my things to go to first. I can normally do the majority of my workout with free weights; I get creative. It definitely helps body image when like I've seen myself increase weight.'

\section{Figure 5}

"My favorite thing to see at the gym" by Amy

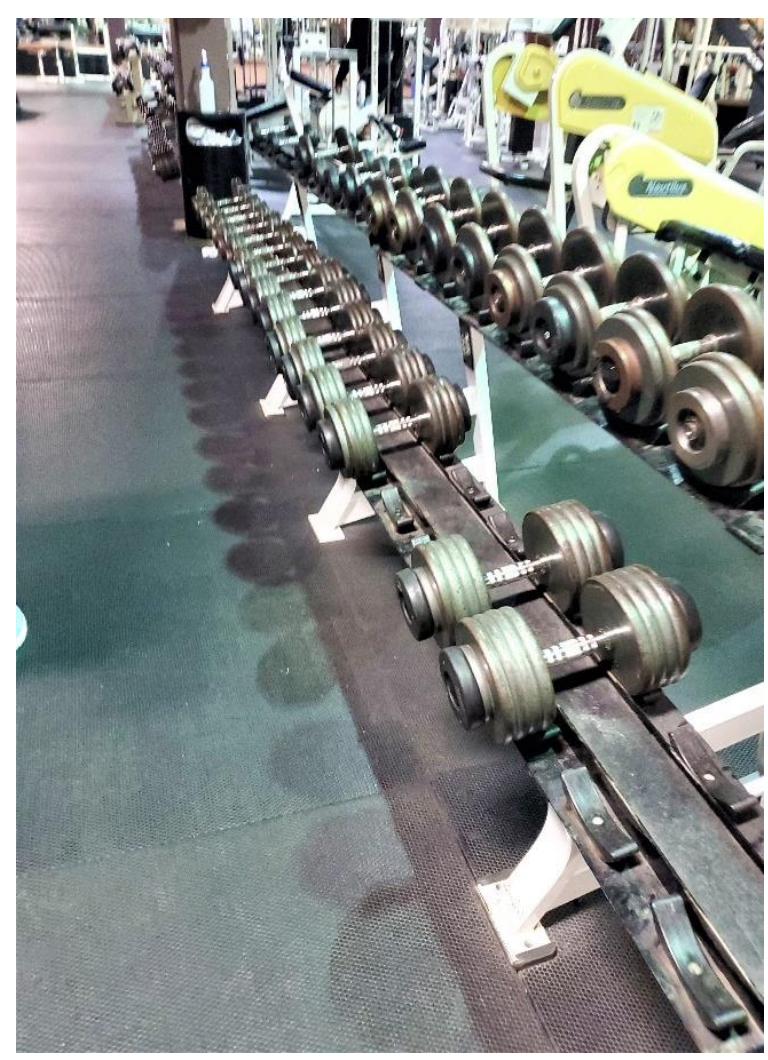

The opportunity to scale workouts and have access to equipment, which fostered the participants' ability to explore their physical abilities, was encouraged through textual messages on decals in some fitness centers. For example, Ali and Bri highlighted the encouraging experience related to message boards on which facility staff wrote a new message each day. The content was described as self-compassionate, which motivated Ali to practice self-kindness towards her body and exercise in ways that positively served her body: '[It means that] I'm at a 
different point than everyone, but like we're [all] here for maybe the same reason, kind of the same reasons, but like we're doing what's best for us.'

Figure 6

"We are [all] doing what's best for us" by Ali

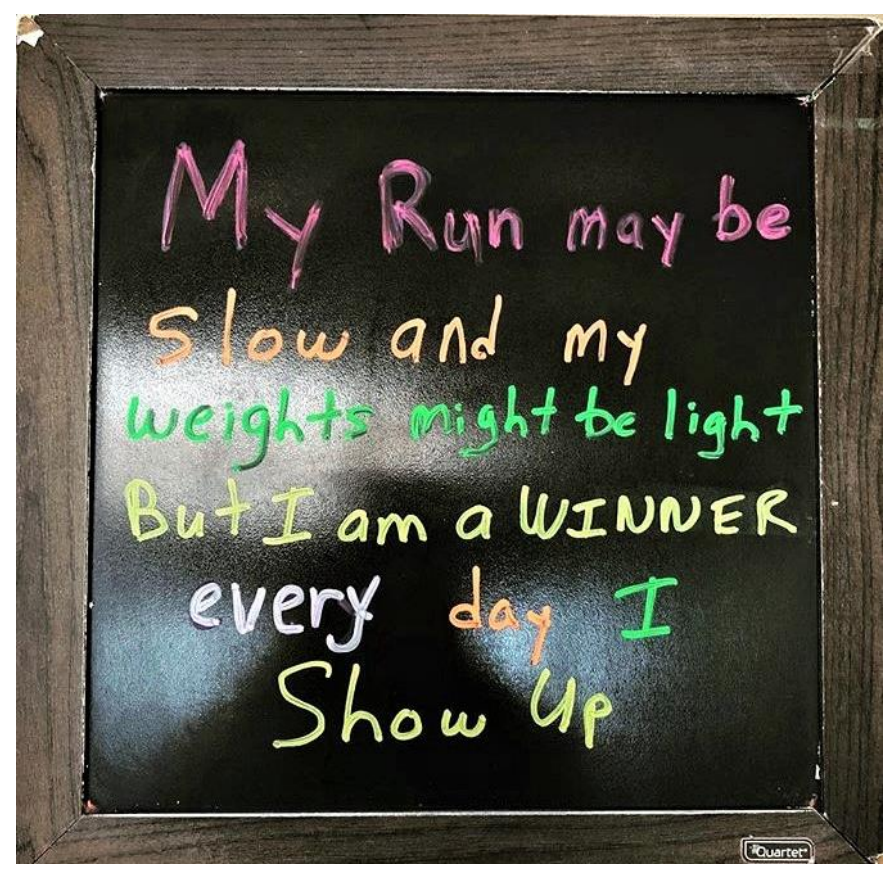

Research exploring the influence of textual messaging in fitness centers suggests that typical language insinuates that if women 'tried harder' or 'pushed themselves', they could achieve the societal definition of fit and attain the ideal (Fisher, Berbary, \& Misener, 2018, p.488).

Unfortunately, Fisher and colleagues highlighted that this type of language often creates conflict for women's experiences with their body. In our study, participants verbalized that language consistent with self-acceptance (e.g., 'Go on, flex!', Sam) and self-kindness (e.g., 'Do more of what makes you happy', Bri), encouraged a more positive and harmonious existence with their body while exercising. All the participants shared that quotations, decals, and message boards which displayed textual messages of gratitude, self-compassion, and self-acceptance helped them believe their fitness centers challenged negative messaging portrayed by the broader fitness 
culture. Strengthening the support for self-compassion-based messaging, Lex described a subspace within her gym (i.e., separate exercise room) adorned with self-compassion messages as 'her favorite place'. Ali and Emi, who participated in fitness centers without mirrors (e.g., CrossFit box, cycle studio), also noted that messaging which fostered a culture of selfcompassion (e.g., 'Head high and proud. This stuff is hard', Emi) de-emphasized appearance and emphasized physical effort and performance (e.g., 'It doesn't matter where you finish, only that you finish with integrity and give it a solid effort', Emi). Self-compassion-based messages presented through platforms such as social media have been shown to buffer the internalization of the fit ideal for young adult college women (Slater, Varsani, \& Diedrichs, 2017). Our results suggested a similar experience whereby messages directly displayed in fitness centers through posters, decals, and message boards, buffered negative body experiences. Thus, despite the dominance of discouraging factors in the fitness center, participants felt that the presence of selfcompassion messages challenged their negative thoughts and feelings about their body while exercising.

In addition to textual messages which encouraged self-compassion and body acceptance, fitness centers that included efforts to moderate apparel standards were perceived as encouraging. For Lex, an enforced dress code minimized the pressure to wear tight, form fitting clothes and encouraged comfort in her own skin: 


\section{Figure 7}

A space for "all body shapes and all body sizes" by Lex

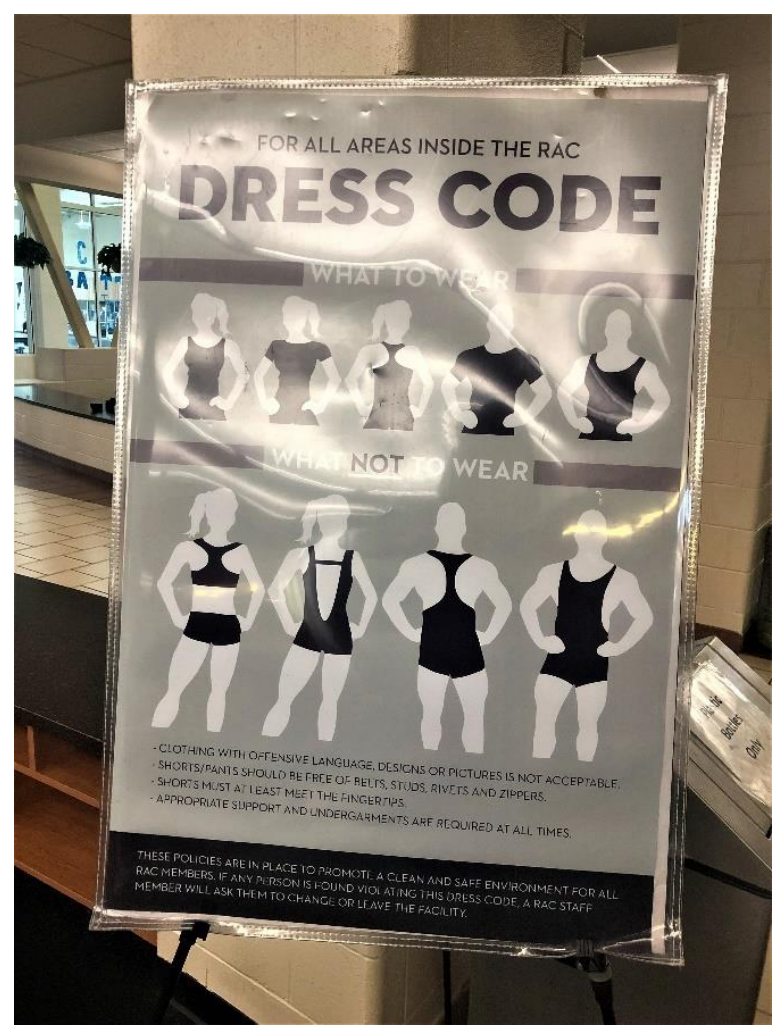

When I see those people I am immediately self-conscious or I'm like you know "I wish I looked like that". I just think [the dress code] is more inclusive to all people....I think that they were just trying to create a space where everyone feels comfortable. I'm ten times more confident knowing I won't see any of the [negative influencers].

Lex elaborated on how clothing played a key role in how she perceived herself and other women in the fitness center. The enforcement of a dress code minimized body comparison for Lex because it encouraged participation of women of all shapes and sizes. Craig and Liberti (2007) described that representation of all women in fitness centers can help promote health as a state of being, not as a particular body type. In our study, participants felt that an established dress code 
in the fitness center encouraged the participation of women of all ages, shapes, and backgrounds, deemphasized a singular body ideal, and supported body acceptance.

\section{Navigating Their 'Place'}

The participants' experience with the discouraging and encouraging factors in their

fitness centers influenced how they used the space itself as well as the meaning they attributed to their bodies while exercising. Several of the participants preferred an exercise environment which supported their freedom to choose exercises and workouts. Unfortunately, most fitness centers are designed to segregate spaces. Therefore, most of the participants described navigating this barrier by seeking out a subspace in the facility characterized, by what they labeled, as 'comfortable'. The word 'comfortable' arose repeatedly across our participants' experiences and, in this context, comfort represented the absence of conflict. In prior research, comfort in womenonly fitness centers is provided by an organizational culture of nonjudgement (Craig \& Liberti, 2007); however, in many mixed-gender fitness centers, a culture of non-judgement is not prioritized. To navigate negative experiences, participants in this study chose to, when possible, exercise in non-threatening spaces to lessen risk of objectification, embarrassment, or judgement. This was predominately achieved by secluding oneself in a private room, free of others. For example, Mya described hiding in the group exercise room during workouts: 


\section{Figure 8}

"Comfortable in this space” by Mya

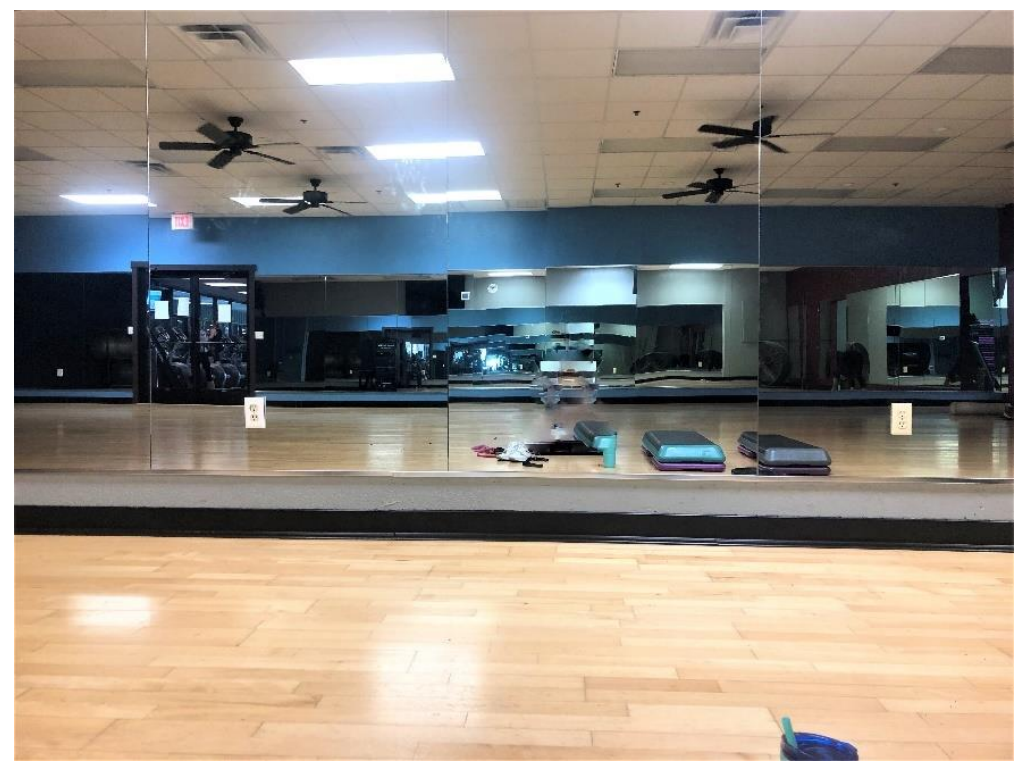

Note: photo editing included blurring using Photoshop to de-identify individual.

You might be doing an exercise that might not be the cutest one, you know glutes, like hip thrusts and stuff are actually - they're pretty awkward to do in front of people. When my face gets really red and I'm like dead-sweating, no one can see and so, it's - I just feel like $100 \%$ in my zone and focused.

Researchers have noted that seeking comfortable subspaces is a common negotiating strategy for women in fitness centers (Fisher, Berbary, \& Misener, 2018). Fisher and colleagues found that negotiating a constraint does not necessarily ensure participant enjoyment or remove the barriers that adult women face. Piran's (2017) DTE describes a dimension of body connection and comfort, the quality of the connection to the body, as either comfortable or problematic. Aligned with Piran's theory, participants in this study experienced salient body disconnection and discomfort in the segregated structure of the weight-area of the fitness center and actively sought spaces which afforded a more positive experience of embodiment, consistent with the body 
connection and comfort dimension of the theory. There were participants who felt discouraged by the need to seek a comfortable subspace in the fitness center, especially when engaging in exercises believed to further objectify women's bodies or go beyond the bounds of acceptable femininity. In contrast, a few participants in our study perceived this negotiating strategy as an opportunity: 'That can be as exciting as you want it to be, but as calm. Sometimes having the empty space to yourself is encouraging; you can do your own thing without feeling judgement or consideration towards anyone else's space' (Eve). This finding suggests that the provision of private spaces in the fitness center may be the encouraging factor that some women need to participate in fitness.

Markula (2003) described exercisers that continue to participate according to gender lines, while also questioning the body ideals promoted by the fitness industry, as conflicted conformists. Because it represents the path of least resistance, many individuals accept and internalize exercise gender norms (Lorber, 2010). The acceptance of gender lines in the weight area ultimately deterred Kay from performing complex exercises that she had learned through years of sports participation. In a hegemonic masculine environment, she felt afraid of taking up space, negatively influencing her confidence in performing mastered exercises: 


\section{Figure 9}

"Maybe they should have it instead of me" by Kay

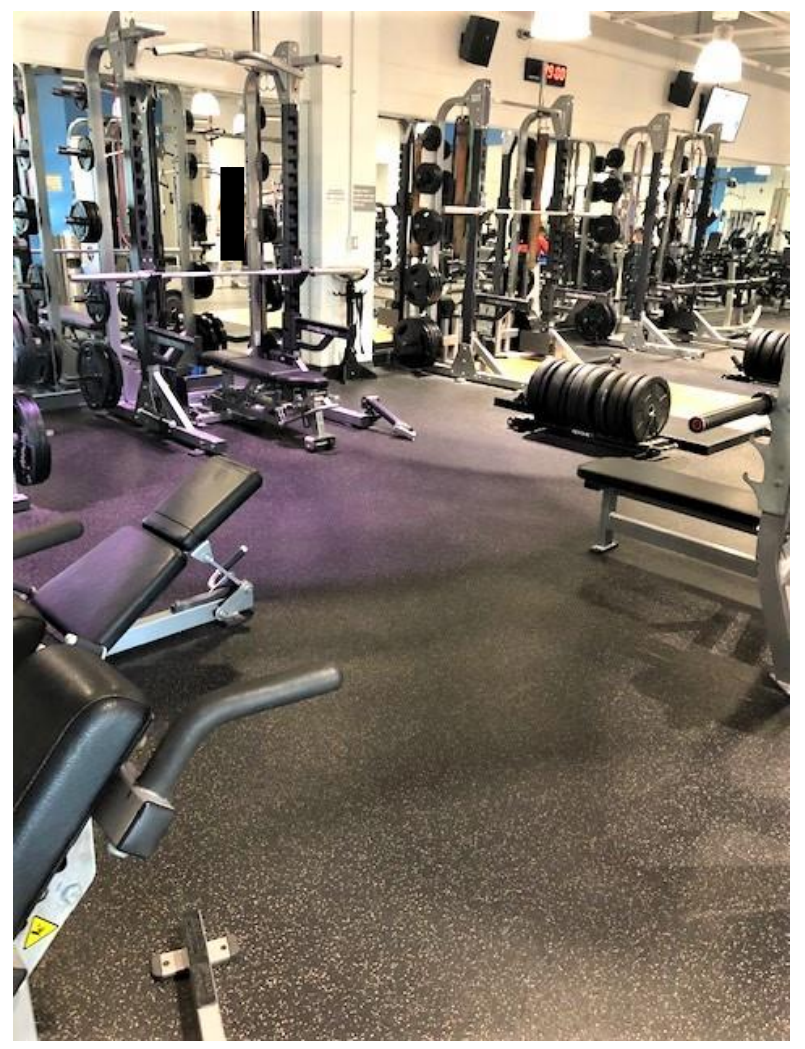

Using platforms and barbells is something I've always been proud of being able to do, just because I had to do it for sports. I tend to actually stay away from them because I feel like they're kind of the center of attention. Everyone is facing that area and I don't want to take up too much of someone else's time if they're waiting for it - especially if they're lifting more than I am so I feel like maybe they should have it instead of me and I can go just use dumbbells or something like that.

The participants in our study changed their exercise routine to adhere to established gender norms by establishing their 'place' in a private subspace of the facility or by avoiding exercises they had mastered. Unfortunately, these spatial practices, like the ones described by our 
participants, further reinforce power relations, gender norms, and the spatial privilege of hegemonic masculinity (Coen, Rosenberg, \& Davidson, 2018). Furthermore, how women use the exercise space is consistent with the agency and functionality dimension of Piran's (2017) DTE. Piran suggests that young adult women who are able to maintain agency in their physical environment, through physical ability and functionality, report more positive embodiment experiences. Restrictions in the exercise space may lead to a restricted physical expression which is associated with the loss of valued experience of competence (Piran, 2017). In this study, barriers to agency and functionality, a critical component of a positive embodiment experience, were represented through Kay's experience of being forced to utilize the fitness center in restrictive ways.

A few of the participants in our study consciously rejected the prescribed gender lines of their fitness center and configured their workouts as demonstrations to advocate for a place for women in the weight areas. For example, Amy navigated her fitness center by entering the maledominated weight area on a regular basis to communicate that women can capably lift weights:

I want to be strong and I want to be independent. I mean, I'm not saying I'm nearly close to outlifting any of those guys, but to know - like when I'm carrying a heavy box and they're like "oh let me take that from you", and I can be like, “no, I'm perfectly capable”.

Amy highlighted that she was often the only woman in the weight area of the gym, which resulted in an uncomfortable experience. However, in contrast to some of the participants, she believed her responsibility as a woman in fitness was to advocate for the representation of women in the weight areas. She did acknowledge that she experienced perceived - and sometimes obvious - social sanctions for her efforts: 
There is a lot of men back there and they're all huge guys. I went up to the bench beside this one guy and he just gives me this funny look, like "what are you doing back here" and I go grab some weight and start lifting while he's still just sitting there like "what are you doing?"

Previous researchers have described this form of advocacy as crossing the gendered lines - the social and structural boundaries separating gendered activities and spaces (Coen, Rosenberg, \& Davidson, 2018). Furthermore, using the body as protest, resistance, and defiance towards 'normative' pressures is suggested by Piran (2017) as a critical component of a positive experience of embodiment, which aligns with one's need for comfort, safety, and joy in one's body. Perhaps this resistance also helps strengthen one's identity as a strong, active person. For our participants, these overt efforts were both empowering and difficult to sustain in the face of embedded masculinity and stereotypes about women in fitness. These results elucidate that women are aware of the genderedness of fitness centers, and while some navigate this disempowering context by seeking comfortable subspaces and avoiding threatening machines, which unintentionally reinforced gender lines, some are using their exercising body to meet personal needs of an embodied exercise experience and advocate for the equal representation of strong women in the male-dominated weight areas of their fitness centers.

\section{Conclusions and Implications}

Using a hermeneutic phenomenological lens, we explored how young adult female exercisers experience their body (e.g., weight, shape, size, appearance) while exercising in fitness centers and the specific elements of their fitness center they perceived to positively, and negatively, influence how they experience their body. Those within the fitness industry claim that fitness centers provide an accessible exercise space for women to engage in activities that 
will increase their health; therefore, fitness centers are marketed as safe, inclusive, and empowering spaces (e.g., Planet Fitness, LA Fitness). Yet, when viewing fitness centers through a sociocultural lens, it became clear that fitness centers may not be experienced in ways they are intended. Research suggests they perpetuate gender expectations and communicate highly prescribed expectations around women's bodies, strength, and abilities (Berbary, 2012; Prichard \& Tiggemann, 2005). 'Doing' exercise is in effect 'doing' gender (Dyck, 2003), and, although not asked about gender explicitly, our participants' experiences bolstered the need to address gender inequities in this context. The participants' body experiences in their fitness centers were heavily influenced by negative factors in their environment. They felt doubt about their sense of belonging in the weight area of the fitness center due to dominance of male musculature on machines, lack of strength machines designed for women, and presence of machines that reinforced gender norms about strength. Unique to our study, encouraging factors present in some of the fitness centers, including self-compassion textual messages and opportunity to scale and choose empowering exercises, helped buffer the self-conscious, threatening experience of exercising in traditional, mixed-gendered fitness centers. The participants' broad range of body experiences map onto several constructs represented in Piran's (2016) DTE, providing perspective on ways women inhabit their bodies and how the body experience can change over time in relation to one's social environment.

There were several strengths of this study. First, Photovoice was a novel method to capture the emotional salience of the participants' body experiences while exercising. Second, this study used a wide range of qualitative methodologies from phone interviews to photographs to virtual conference interviews and member reflections, which allowed for complexities and nuances in each of the participants' stories to be highlighted in rich detail. Finally, we suggest 
further exploration of sociocultural frameworks for understanding the intrapersonal, social, and cultural supports and barriers that young adult women experience in a context dominated by hegemonic masculinity. Thus far, much of the research on body experiences of women in fitness centers has been founded in self-objectification theory (Fredrickson \& Roberts, 1997). However, our results support the need to expand the scope of inquiry to include social and cultural influences as has been done in the sport context (i.e., Petrie \& Greenleaf, 2012; Voelker \& Reel, 2015) as well as embodiment theories which explore the relationship between embodied practices and social structures (i.e., Piran, 2017). Exploring these phenomena through a theoretical lens can serve as basis for understanding the levels of complexity related to body experiences of young adult women.

We acknowledge the current study had limitations. First, this study was conducted solely in mixed-gendered gyms. Women-only fitness centers have been suggested to allow women to experience fitness free from hegemonic masculinity (Wearing, 1998). However, Fisher, Berbary, and Misener (2018) cautioned against the growth of women-only fitness centers, as they may further reinforce gender lines in the fitness culture. Nonetheless, this study calls for future research exploring the body experiences of women in women-only fitness centers using similar methodologies to explore similarities and differences in this context. Second, while the focus of this study was on the experiences of women, many men may also feel constrained within the gender normative space of some fitness centers, as they too are held to gendered standards of the culturally dominant, hegemonic masculine ideal (Coles, 2009). Finally, comparative studies with women of different age groups (e.g., adult, older adults), different ethnicities, and at different levels of experience in a fitness center (e.g., beginner exercisers) can broaden our understanding of the encouraging and discouraging body experiences of women in fitness. 
Exercise has the potential to enhance a woman's connection to her body. By forming body knowledge through fitness, one can learn to understand ambiguous feelings, trust their own body, develop competence, and improve confidence through an embodiment experience (Parviainen \& Aromaa, 2017). However, in order to accomplish this, our study suggested that fitness centers must take deliberate action to change the structural and cultural standards of their facilities.

\section{References}

Andreasson, J., \& Johansson, T. (2014). The fitness revolution. Historical transformations in the global gym and fitness culture. Sport Science Review, 23(3-4), 91-111. doi:10.2478/ssr2014-0006

Barbells. (2020). In Rogue.com. Retrieved from https://www.roguefitness.com/weightliftingbars-plates/barbells

Benner, P. E., \& Wrubel, J. (1989). The primacy of caring: Stress and coping in health and illness. Addison-Wesley/Addison Wesley Longman.

Berbary, L. A. (2012). “Don’t Be a Whore, That's Not Ladylike” Discursive Discipline and Sorority Women's Gendered Subjectivity. Qualitative Inquiry, 18(7), 606-625. Doi: $10.1177 / 1077800412450150$

Biddle, S. J., \& Mutrie, N. (2007). Psychology of physical activity: Determinants, well-being and interventions. Routledge.

Boepple, L., Ata, R. N., Rum, R., \& Thompson, J. K. (2016). Strong is the new skinny: A content analysis of fitspiration websites. Body image, 17, 132-135. doi:10.1016/j.bodyim.2016.03.001 
Bordo, S. (2004). Unbearable weight: Feminism, Western culture, and the body. University of California Press.

Campbell, A., \& Hausenblas, H. A. (2009). Effects of exercise interventions on body image: A meta-analysis. Journal of health psychology, 14(6), 780-793.

Cash, T. F. (2004). Body image: Past, present, and future. Body Image, 1, 1-5. doi:10.1016/S1740-1445(03)00011-1

Cash, T. F. (2012). Encyclopedia of body image and human appearance. Academic Press.

Chang, C. C., Pan, M. C., \& Shu, S. T. (2018). Relationship between self-presentation, exercise dependence, and perceived body image. International Journal of Sport and Exercise Psychology, 1-13. doi:10.1080/1612197X.2018.1444078

Cockburn, C., \& Clarke, G. (2002). “Everybody's looking at you!”: Girls negotiating the "femininity deficit" they incur in physical education. In Women's Studies International Forum (pp. 651-665). Pergamon.

Coen, S. E., Rosenberg, M. W., \& Davidson, J. (2018). “It's gym, like gym not Jim”: Exploring the role of place in the gendering of physical activity. Social Science \& Medicine, 196, 29-36. doi: 10.1016/j.socscimed.2017.10.036

Coles, T. (2009). Negotiating the field of masculinity: The production and reproduction of multiple dominant masculinities. Men and masculinities, 12(1), 30-44. doi: $10.1177 / 1097184 X 07309502$

Craig, M. L., \& Liberti, R. (2007). “Cause That's What Girls Do” The Making of a Feminized Gym. Gender \& Society, 21(5), 676-699. doi: 10.1177/0891243207306382

Discouraging. (2020). In Merriam-Webster.com dictionary. Retrieved from https://www.merriam-webster.com/dictionary/discouraging 
Dworkin, S. L. (2003). A woman's place is in the... cardiovascular room? Gender relations, the body, and the gym. In A. Bolin \& J. Granskog (Eds.). Athletic intruders: Ethnographic research on women, culture, and exercise, 131-158. Suny Press.

Dyck, I. (2003). Feminism and health geography: twin tracks or divergent agendas?. Gender, place and culture, 10(4), 361-368. doi: 10.1080/0966369032000153331

Encouraging. (2020). In Merriam-Webster.com dictionary. Retrieved from https://www.merriam-webster.com/dictionary/encouraging

Fisher, M. J. R., Berbary, L. A., \& Misener, K. E. (2018). Narratives of negotiation and transformation: Women's experiences within a mixed-gendered gym. Leisure Sciences, 40(6), 477-493. doi: 10.1080/01490400.2016.1261744

Fredrickson, B. L., \& Roberts, T. A. (1997). Objectification theory: Toward understanding women's lived experiences and mental health risks. Psychology of women quarterly, 21(2), 173-206. doi:10.1111/j.1471-6402.1997.tb00108.x

Furnham, A., Badmin, N., \& Sneade, I. (2002). Body image dissatisfaction: Gender differences in eating attitudes, self-esteem, and reasons for exercise. The Journal of Psychology, 136(6), 581-596. doi:10.1080/00223980209604820

Heidegger, M. (1962). In J. Macquarrie \& E. Robinson (Eds.). Being and time. Oxford: Blackwell.

Kerry, D. S., \& Armour, K. M. (2000). Sport sciences and the promise of phenomenology: Philosophy, method, and insight. Quest, 52(1), 1-17. doi: $10.1080 / 00336297.2000 .10491697$

Klein, A. M. (1993). Little big men: Bodybuilding subculture and gender construction. Suny Press. 
Laverty, S. M. (2003). Hermeneutic phenomenology and phenomenology: A comparison of historical and methodological considerations. International journal of qualitative methods, 2(3), 21-35. doi:10.1177/160940690300200303

Le Vasseur, J. J. (2003). The problem of bracketing in phenomenology. Qualitative Health Research, 13, 408-420. doi: 10.1177/1049732302250337

Lorber, J. (2010). Feminisms and their contributions to gender equality. Gender Inequality: Feminist Theories and Politics, 1-20.

Markula, P. (2003). Post-modern aerobics: Contradiction and resistance. In A. Bolin \& J. Granskog (Eds.). Athletic intruders: Ethnographic research on women, culture, and exercise, (pp. 53-78). Suny Press.

Miller, A. E. J., MacDougall, J. D., Tarnopolsky, M. A., \& Sale, D. G. (1993). Gender differences in strength and muscle fiber characteristics. European journal of applied physiology and occupational physiology, 66(3), 254-262. doi: 10.1007/BF00235103

Munhall, P. L. (1989). Philosophical ponderings on qualitative research methods in nursing. Nursing Science Quarterly, 2(1), 20-28. doi:10.1177/089431848900200109

Neighbors, L. A., \& Sobal, J. (2007). Prevalence and magnitude of body weight and shape dissatisfaction among university students. Eating behaviors, 8(4), 429-439. doi: 10.1016/j.eatbeh.2007.03.003

Noll, S. M., \& Fredrickson, B. L. (1998). A mediational model linking self-objectification, body shame, and disordered eating. Psychology of Women Quarterly, 22(4), 623-636. doi: 10.1111/j.1471-6402.1998.tb00181.x 
Nykiforuk, C. I., Vallianatos, H., \& Nieuwendyk, L. M. (2011). Photovoice as a method for revealing community perceptions of the built and social environment. International Journal of Qualitative Methods, 10(2), 103-124. doi:10.1177/160940691101000201

Parviainen, J., \& Aromaa, J. (2017). Bodily knowledge beyond motor skills and physical fitness: a phenomenological description of knowledge formation in physical training. Sport, Education and Society, 22(4), 477-492. doi: 10.1080/13573322.2015.1054273

Patton, C. (2001). "Rock hard" judging the female physique. Journal of sport and social issues, 25(2), 118-140. doi: 10.1177/0193723501252002

Petrie, T. A., \& Greenleaf, C. (2012). Body image and sports/athletics. In Encyclopedia of body image and human appearance (pp. 160-165). Academic Press.

Pietkiewicz, I., \& Smith, J. A. (2014). A practical guide to using interpretative phenomenological analysis in qualitative research psychology. Psychological Journal, 20(1), 7-14. doi:10.14691/CPPJ.20.1.7

Piran, N. (2016). Embodied possibilities and disruptions: The emergence of the experience of embodiment construct from qualitative studies with girls and women. Body Image, 18, 43-60. doi: 10.1016/j.bodyim.2016.04.007

Piran, N. (2017). Journeys of embodiment at the intersection of body and culture: The developmental theory of embodiment. Academic Press.

Plunkett, R., Leipert, B. D., \& Ray, S. L. (2013). Unspoken phenomena: using the photovoice method to enrich phenomenological inquiry. Nursing Inquiry, 20(2), 156-164. doi:10.1111/j.1440-1800.2012.00594.x 
Ponterotto, J. G. (2005). Qualitative research in counseling psychology: A primer on research paradigms and philosophy of science. Journal of Counseling Psychology, 52(2), 126. doi:10.1037/0022-0167.52.2.126

Prichard, I. J., \& Tiggemann, M. (2005). Self-objectification, body image and reasons for exercise. In Past reflections, future directions: 40th APS Annual Conference. Australian Psychological Society.

Prichard, I., \& Tiggemann, M. (2012). Predictors of self-objectification in new female fitness center members. Women in Sport and Physical Activity Journal, 21(1), 24-32. doi: doi.org/10.1123/wspaj.21.1.24

Reel, J. J., Greenleaf, C., Baker, W. K., Aragon, S., Bishop, D., Cachaper, C., ... \& Hattie, J. (2007). Relations of body concerns and exercise behavior: A metaanalysis. Psychological reports, 101(3), 927-942. doi: 10.2466/pr0.101.3.927-942

Robinson, L., Prichard, I., Nikolaidis, A., Drummond, C., Drummond, M., \& Tiggemann, M. (2017). Idealised media images: The effect of fitspiration imagery on body satisfaction and exercise behaviour. Body image, 22, 65-71. doi: 10.1016/j.bodyim.2017.06.001

Sassatelli, R. (1999). Interaction order and beyond: A field analysis of body culture within fitness gyms. Body \& Society, 5(2-3), 227-248. doi: 10.1177/1357034X99005002013

Silverstein, B., Peterson, B. and Perdue, L. (1986). Some correlates of the thin standard of bodily attractiveness for women. International Journal of Eating Disorders, 5, 895-905.

Slater, A., \& Tiggemann, M. (2006). The contribution of physical activity and media use during childhood and adolescence to adult women's body image. Journal of Health Psychology, 11(4), 553-565. doi:10.1177/1359105306065016 
Slater, A., Varsani, N., \& Diedrichs, P. C. (2017). \# fitspo or\# loveyourself? The impact of fitspiration and self-compassion Instagram images on women's body image, selfcompassion, and mood. Body Image, 22, 87-96. doi: 10.1016/j.bodyim.2017.06.004

Smith, Flowers, \& Larkin (2009). Interpretative Phenomenological Analysis: Theory, method and research. Thousand Oaks, CA: Sage Publications.

Smith, B., \& McGannon, K. R. (2018). Developing rigor in qualitative research: Problems and opportunities within sport and exercise psychology. International review of sport and exercise psychology, 11(1), 101-121. doi:10.1080/1750984X.2017.1317357

Stern, M. (2008). The Fitness Movement and the Fitness Center Industry, 1960-2000. Business \& Economic History On-Line, 6.

Strelan, P., Mehaffey, S. J., \& Tiggemann, M. (2003). Brief report: Self-objectification and esteem in young women: The mediating role of reasons for exercise. Sex Roles, 48(1-2), 89-95. doi:10.1023/A:1022300930307

Taylor, C. (1985). Human agency and language. Cambridge University Press.

Tiggemann, M., \& Lynch, J. E. (2001). Body image across the life span in adult women: The role of self-objectification. Developmental psychology, 37(2), 243. doi:10.1037/00121649.37.2.243

Tiggemann, M., \& Williamson, S. (2000). The effect of exercise on body satisfaction and selfesteem as a function of gender and age. Sex Roles, 43(1-2), 119-127. doi:10.1023/A:1007095830095

Voelker, D. K., \& Reel, J. J. (2015). An inductive thematic analysis of female competitive figure skaters' experiences of weight pressure in sport. Journal of Clinical Sport Psychology, 9(4), 297-316. doi:10.1123/jcsp.2015-0012 
Voelker, D. K., Reel, J. J., \& Greenleaf, C. (2015). Weight status and body image perceptions in adolescents: current perspectives. Adolescent health, medicine and therapeutics, 6, 149. doi:10.2147/AHMT.S68344

Wang, C. C. (1999). Photovoice: A participatory action research strategy applied to women's health. Journal of Women's Health, 8(2), 185-192. doi:10.1089/jwh.1999.8.185

Wang, C. C., \& Burris, M. A. (1994). Empowerment through photo novella: Portraits of participation. Health Education Quarterly, 21(2), 171-186.

doi:10.1177/109019819402100204

Wang, C. C., \& Burris, M. A. (1997). Photovoice: Concept, methodology, and use for participatory needs assessment. Health education \& behavior, 24(3), 369-387. doi:10.1177/109019819702400309

Wearing, B. (1998). Leisure and feminist theory. Sage.

Williams, P. A., \& Cash, T. F. (2001). Effects of a circuit weight training program on the body images of college students. International Journal of Eating Disorders, 30(1), 75-82. doi:10.1002/eat.1056

Yardley, L. (2008). Demonstrating Validity in Qualitative Psychology. In J. A. Smith (Eds.), Qualitative psychology: A practical guide to research methods (pp. 235-251). Sage Publications. 


\section{Appendix A: Extended Introduction}

\section{Basic Assumptions}

- The participants can use, and have access to, mobile, smartphone devices with appropriate camera features to complete the photography phase of the study.

- The two-week photography phase of the study was an adequate amount of time for capturing the breadth of participants' lived experiences (Nykiforuk, Vallianatos, \& Nieuwendyk, 2011).

- The PhotoVoice method was an appropriate data collection method to apply to a novel physical activity context, like fitness centers, as it has been applied to research exploring the relationship between environment and physical activity engagement (e.g., Belon, Nieuwendyk, Vallianatos, \& Nykiforuk, 2014) as well as investigations on sensitive personal topics, such as body image (see Brunsden \& Goatcher, 2007).

- The interview guide appropriately elicited honest, reflective, and accurate responses from the participants (Wang, 1999). In addition, photo-elicitation during the interview process supported participants' recall of salient environmental and intrapersonal elements relevant to the study's purpose.

- The women included in the study have conscious awareness of their body image while in the exercise setting and engage is self-objectification in the exercise context (Tiggemann \& Williamson, 2000).

\section{Limitations of the Study}

- Descriptions and interpretations of the phenomenon central to this study were limited to the experiences of the 11 young adult women who volunteered for the study. Therefore, the intentional application of an Interpretative Phenomenology Analysis approach will 
share the stories of these participants rather than propose generalizations about body image experiences of women more broadly (Smith, Harré, \& Van Langenhove, 1995).

- Hermeneutic phenomenology maintains that separating the researcher from the interpretative process is an impossibility. Hence, the co-construction of meaning and essence between participant and researcher suggests that the researcher's interpretation based on personal social and cultural experiences may influence the results (Ponterotto, 2005) and full bracketing was not a possibility.

- Given the sensitive nature of the topic of body image among young women, it is possible the participants were resistant or filtered their responses during the interview process. Attempts were made to build rapport, use appropriate self-disclosure, and provide ongoing resources (e.g., National Suicide Prevention Lifeline, National Eating Disorders Association Helpline).

- Interview content was limited to the number of photographs participants choose to discuss during the interviews which may mean that a subset of captured photographs were not included in the dataset or interpretation.

- The PhotoVoice approach to data collection only captured objective, visible features of the fitness center environment which may have minimized the inclusion of more subjective, nuanced factors that cannot be reasonably captured with photography. However, the interview guide used included an additional subset of questions (e.g., What additional factors in your fitness center, that were not captured in the photographs, are encouraging to your body image while exercising? Discouraging?) to minimize this limitation. 


\section{Definition of Terms}

- Body Image: a multidimensional construct which encompasses self-perceptions and attitudes about one's physical appearance, including overall appearance and/or specific physical characteristics such as weight or body shape through thoughts, feelings, beliefs, and behaviors (Cash, 2004).

- Positive Body Image: refers to the act of accepting and appreciating one's body, including its unique characteristics, functionality, and capabilities and is distinct from the absence of body image dissatisfaction (Tylka, 2011).

- Negative Body Image: refers to dysphoric body image emotions (e.g., anxiety, dissatisfaction, dejection) which are, in part, influenced by contextual situations involving body exposure, social scrutiny, social comparisons, certain clothing, presence of mirrors, weighing, exercising, and so forth (Cash, 2004).

- Fitness Center: a multidimensional space where a variety of specialized equipment and exercise modalities are provided in one facility for the purpose of structured physical activity.

- Encourager: for the purposes of this study, encouragers will be defined as factors which "give someone support or confidence; positive and giving hope for the future" (Dictionary, n.d.).

- Discourager: for the purposes of this study, discouragers will be defined as factors “causing someone to lose confidence or enthusiasm" (Dictionary, n.d.).

- Embodiment: a process of inhabiting the body that provides a close, connected, and intimate relationship with the body through which one can effectively know, respect, and voice bodily experiences and needs (Piran, 2002; 2015). An embodied individual 
experiences their body as a vital and integral part of their self-expression and power, and as central to their overall well-being (Mahlo \& Tiggemann, 2016). 


\section{Appendix B: Extended Methods}

\section{Interpretative Phenomenological Study Design}

The current study used an Interpretative Phenomenological Analysis approach using a PhotoVoice method to describe young female exerciser's experiences of their body and body image in fitness centers. In this section, phenomenology as a philosophy and method to research, the PhotoVoice approach as primary data collection within the paradigm, the participants and setting, and procedures used for the study will be outlined and described.

Phenomenology as a theoretical lens. Historically, academic research has predominantly been driven by the investigation of the observable (Laverty, 2003). Phenomenology, developed from the philosophical work of Edmund Husserl (1952/1980), evolved when qualitative scholars questioned positivism and the quest to identify the 'objective' truths of human behavior. At the time, phenomenology was an avant-garde idea which, as AllenCollinson described, sought to study the phenomena of human existence and how phenomena are perceived in consciousness (as cited in Smith \& Sparkes, 2016). Phenomenologists, with Husserl at the forefront, argued that humans should be acknowledged "as open and engaged beings capable of developing personal meaning in the process of actively manifesting themselves" (Meier, 1988, p. 92). Husserl defined phenomenology as a rigorous social science focused on developing rich detailed descriptions of lived-experiences or 'phenomena' (Allen-Collinson, 2011). Within phenomenology, four main threads have been proposed including realist, transcendental constitutive, hermeneutic, and existential phenomenology all of which are defined by distinctive perspectives, or 'persuasions' (Embree \& Mohanty, 1997). Phenomenology as a philosophy continues to evolve and is far from being 'resolved' due to the different ontological and epistemological foundations of each thread (Allen-Collinson, 2011). When taken together, 
phenomenology represents a complex and nuanced perspective of the human condition. Despite the complex nature of phenomenology, it should be emphasized that underlying each persuasion is a worldview grounded in the essence of the lebenswelt (life world), or lived experience (van Manen, 1997).

Hermeneutic phenomenology. I am working from a hermeneutic phenomenology tradition. To maintain the rigor needed to ethically use methodologies that align with this orientation (Kerry \& Armour, 2000), it is important I acknowledge the similarities and differences between Husserl's seminal work in phenomenology and hermeneutic phenomenology, which was put forth by his successor, Heidegger (1927/1962). Like phenomenology, hermeneutic phenomenology is concerned with the human experience as it is lived. However, where Husserl and Heidegger diverge is in how they believe the lived experience unfolds (Laverty, 2003). For example, Husserl's phenomenology focuses on understanding the phenomenon itself whereas Heidegger's hermeneutic phenomenology focuses on dasein, or 'the situated meaning of a human in the world'. In other words, hermeneutic phenomenology is interested in historicality of lived experiences and understanding a person's situatedness in the phenomenon (Laverty, 2003). Therefore, how a person understands their lived experience, and the world, is undeniably enmeshed with their historical, cultural, and social contexts (Munhall, 1989). Within the context of the current research, hermeneutic phenomenology holds that it is critical to understand a young adult woman's social, contextual, and cultural experiences if we are to describe how she understands her body and negotiates her body image while exercising. To truly understand a lived experience within the philosophy of hermeneutic phenomenology, detailed descriptions of an individual's background, preunderstandings, co-constitution, and interpretation are paramount (Benner \& Wrubel, 1989). 
A person's background is defined as a part of a person, imparted on them by culture from birth. These personal histories are integral in understanding how humans experience similar phenomena in very different ways (Kerry \& Armour, 2000). The term, pre-understandings, was used by Heidegger to define the structure of a culture as it exists, prior to a person's interaction with, and interpretation of, that culture. As Koch (1995) states, culture is not something that can be 'bracketed' as it is already present in languages, customs, and practices. Co-constitution relates to pre-understandings as it highlights the indisputable link between the "person and the world'. This unity was acknowledged by Heidegger (1927/1962) to mean that "we are constructed by the world in which we live, and, at the same time, we perceive the world from our own experience and background" (Kerry \& Armour, 2000, p. 6). Finally, Heidegger (1927/ 1962) argues that every social interaction involves interpretation based on an individual's background and pre-understanding. Humans are self-interpretative beings, constantly redefining the self through acts of interpretation of the culture in which they exist (Taylor, 1985). In the context of this study, capturing the essence of a young adult woman's experience of body and body image during exercise must acknowledge (1) the social, historical, and structural culture of the fitness center environment, (2) the social, historical, and contextual culture of her life, and (3) her interpretation and meaning-making of her exercising body.

The role of the body in the social world. Hermeneutic phenomenology maintains a strong focus on the social construction of an individual's reality. For scholars researching kinesthetic experiences, the work of existentialist Merleau-Ponty (2001) and sociologicalphenomenologists, such as Berger and Luckmann (1991), is critical for understanding mind-body phenomena in social contexts. Merleau-Ponty's (2001) work discussed the body's role in perception and society and highlighted the importance of bodily intentionality - perceiving the 
body as an attitude towards a task (Allen-Collinson, 2016). In the context of exercise and sport, his constructs have been used to study yoga practice (e.g., Morley, 2001), the sensory experience of the 'sporting body' (e.g., Hockey \& Allen-Collinson, 2007), and 'doing endurance' in women's distance-running (e.g., Hockey \& Allen-Collinson, 2016). In parallel, sociologicalphenomenology suggests that the "body/consciousness/world are all fundamentally connected, braided, interrelating, and mutually influencing” (p. 11, Smith \& Sparkes, 2016). This concept developed from the work of Berger and Luckmann (1966) on the social construction of reality, later evolving into contemporary social constructionism (McCarty \& Schwandt, 2000). Social constructionism ascertains the existence of multiple, subjective, valid realities (Ponterotto, 2005) rather than a singular observable truth. Furthermore, the paradigm contends that individuals construct and define their 'truth' through interactions with others and the historical, social, and cultural environments in which they live (Creswell, 2007; Ponterotto, 2005). In the current study, understanding the exercising body, and resultant body image constructions, cannot ignore that individuals are social interpreters, both constructing and sustaining their body image and exercise behaviors through negotiations within their fitness center environment.

Phenomenology as a methodology. Phenomenology in sport and exercise science has traditionally been used as a method and approach to inquiry. However, phenomenology tradition states that the philosophy should be emphasized as an orientation, rather than a set of procedures (Kerry \& Armour, 2000). If operating within the orientation, phenomenological methodology is a promising approach for the field of sport and exercise (Kerry \& Armour, 2000). Smith and Sparkes (2016) described the characteristics of the phenomenological method to include: 
- Description: when exploring phenomenon within a social or cultural context, we acknowledge the influence of our personal (i.e., the researcher) social and cultural influences on the description of the phenomenon

- Intentionality: the act of directed consciousness which brings the phenomenon into focus

- Epoche: within social constructionism and sociological phenomenology, full epoche, or bracketing of personal history is accepted as an impossibility

- Essences: seeking the essence or core meaning of a phenomenon

As a methodology, phenomenology is useful to not only describe but capture to complex, dynamic body-related experiences of young adult female exercisers, while honoring the presence of the social, cultural, and contextual histories of the participants and researcher.

Researcher positionality. Hermeneutic phenomenology, and social constructionism within that, aligns with the primary researcher's relativist ontological position which suggests that the existence of multiple, valid realities is further interpreted through the co-construction of experience in the participant-researcher exchange (Ponterotto, 2005). This emphasis on coconstruction and the impossibility of complete 'bracketing', or epoche, makes it necessary to examine my background and personal cultural and socio-structural position as the primary researcher and interviewer. The explicit statement of researcher positionality is intentional as it is critical to understand how my interpretations may be shaped by my past experiences. I participated in elite gymnastics for 15 years, during which I was acutely aware of my body weight, shape, size, and appearance. This experience included significant body image disturbances which, after retirement, motivated me to begin exercising in a fitness center with appearance-focused goals and to lose weight. For the following eight years, exercising in a fitness center only exacerbated my body image disturbances as I consistently engaged in social 
comparison, internalized unrealistic body ideals, and exercised purely for appearance-related objectives. Acknowledging this experience and being mindful of the potential influence throughout the research process was paramount. I personally have salient and formative body image-related exercise history, of which I am acutely aware. This 'insider' experience is valuable because it allowed me as the researcher, and interviewer, to connect with the study participants, and deepen questioning and reflection in the interview and analysis process. However, based on my personal history, it is critical to also acknowledge potential assumptions that all women experience body image disturbances, that all women are aware of social body ideals, and that all women experience the fitness culture in a similar way to myself.

In addition to my historical experience as a potential 'insider' of the phenomenon central to this study, it is important to acknowledge my current positioning. More specifically, my training in post-secondary education in clinical mental health, trauma therapy, exercise psychology, and being a researcher of body-image related phenomena inspired a personal process in which my relationship with body, in relation to exercise, has evolved. From this training, I have been able to become self-aware and engage in a self-reflective process through exercise. For example, my relationship with exercise now represents an act of self-kindness in which I am motivated to participate because I feel empowered. Therefore, given the literature suggesting that most young women experience body image concerns (e.g., Pruis \& Janowsky, 2010), my current relationship with exercise may suggest that I am an 'outsider' to the participants included in this study. More specifically, my current awareness of weight pressures within the fitness setting has afforded me the ability to minimize their influence over my body image and self-confidence related to my body weight, shape, size, and appearance. I, therefore, may have been perceived as an outsider to participants who's experiences with weight pressures 
differ from my own. With this, the assumptions that all women have experienced a similar evolution in their relationship to exercise or that all women will experience a similar evolution was acknowledged and minimized. Merriam et al., (2001) suggest that "what an insider 'sees' and 'understands' will be different from, but as valid as out an outsider 'sees"' (p. 415) and argues that both perspectives are equally valid and valuable. Therefore, although my positionality to the current study required rigorous examination of my personal assumptions, my intention, and deliberate constant reflection, to use my personal experience added value to the research process.

Interpretative Phenomenological Analysis. The current study used an interpretative phenomenological analysis (IPA) approach, which is a practical methodology drawing upon the foundational principles of phenomenology, hermeneutics, and idiography (Pietkiewicz \& Smith, 2014). IPA is used to develop an understanding of a lived experience through first-person accounts (Gentles, Charles, Ploeg, \& McKibbon, 2015) and has been applied widely within sport and exercise psychology (see Allen-Collinson, 2016). Through the development of detailed descriptions of a phenomenon, rich insight into how individuals make meaning from their experiences, and their contexts, can be achieved. IPA is consistent with the current study's objectives as the approach assumes individuals are actively engaged in interpreting their concept of self from their experiences and environments (Taylor, 1985), further incorporating key tenets of hermeneutic phenomenological philosophy (Pietkiewicz, \& Smith, 2014). IPA is also idiographic as it includes in-depth analysis of single cases in order to examine individual perspectives of the phenomenon. This provides the researcher the ability to present specific statements about participants rather than propose generalizations (Smith, Harré, \& Van 
Langenhove, 1995). It is through IPA that the current study explored the exercising bodies of young adult women and their body image during exercise in fitness centers.

PhotoVoice approach within phenomenology. Nykiforuk, Vallianatos, and Nieuwendyk (2011) provide a detailed description and procedure of the PhotoVoice method used in the current study. The PhotoVoice approach represents a blended process of photography and narratives to explore social issues (Wang \& Burris, 1994; 1997). The approach has three complimentary goals: (1) provide a platform for participants' lived experiences and viewpoints, (2) involve both the culture and community to exchange ideas surrounding key themes that emerge via photographic art and oral exploration, and (3) ensure that the information is not confined to the academic and scientific communities (Wang and Burris 1994; 1997). PhotoVoice was well-suited for the current research question as it allowed the researcher to richly describe and visualize women's perceptions of their everyday realities. More specifically, PhotoVoice addressed two main goals of the current study: (1) enabled women to document, with personal expression, factors in fitness centers that are integral in their interpretation of their body and body image experiences during exercise and (2) promoted in-depth dialogue in discussions focused on obtaining rich descriptions of the women's lived experiences interacting with those factors in real time.

\section{Study Setting}

This study included participants from community-based fitness centers within the United States. Contemporary fitness culture is largely influenced by the historical development of bodybuilding and the aerobic fitness revolution (Andreasson \& Johansson, 2014). In the 1990's, traditional male-dominated bodybuilding and weight-training gyms shifted to multidimensional fitness gyms where different modalities of exercise were provided in one facility. Prior to this 
global shift, fitness centers were highly gendered but as fitness centers expanded, women began to integrate into the strength-training areas of the gym. In the 1980's and 90's, in what is known as the third-generation fitness wave, commercial fitness centers grew exponentially on a global scale. It was during this time that the general trend of fitness as a mass leisure activity was developed (Andreasson \& Johansson, 2014). This evolution resulted in a fitness center subculture that is founded on the message that a healthy lifestyle, consisting of health, beauty, and youth (i.e., body ideals which are perpetuated by the broader culture), are attainable through structured, deliberate, and routine physical sculpting.

Fitness centers generally house a variety of equipment designed for the purpose of repetitive physical exercise. Many facilities are composed of (a) a main workout area consisting of free weights, dumbbells, barbells, weighted machines, cable-assisted mechanisms, racks and rigs, benches, and stands; (b) a cardiovascular-training area consisting of machines such as ergometric rowers, stationary bikes, elliptical, treadmills, and stair mills; (c) group exercise rooms where organized group classes are led by a qualified instructor; and, occasionally, (d) functional training area with agility-based, non-conventional exercise equipment. Where individual commercial fitness centers differ is in their approach to fitness and their "culture" or "aura of exclusivity" used for attracting their target population (Andreasson \& Johansson, 2014, p. 107). For example, the popular fitness chain Planet Fitness utilizes the slogan "Welcome to Planet Fitness. The Judgement Free Zone ${ }^{\circledR}$ " with the goal of providing a welcoming environment free of body ideals, social comparison, and objectification for all patrons (Planet Fitness, 2019). Conversely, one of the founding commercial fitness centers, Gold's Gym, has a 50-year history that is purported in the media to be heavily influenced by bodybuilding and physique-focused motivations for exercise (Heffernan, 2015). Other fitness centers (e.g., LA 
Fitness) are claimed to advertise to middle- and upper-class America by providing exclusive health and wellness services (Grisso, 2018). At the community level, the YMCA of the USA is a non-profit, community-centered, organization that integrates fitness, exercise, and other physical activities into their mission of strengthening the community (YMCA of the USA, n.d.). In sum, commercial fitness centers vary across many social factors including the culture of their facility. The lived experience of young women and their exercising body in the context of these similar, and yet distinct, social contexts was of interest in the current study.

\section{Recruitment}

Participants were 11 young adult women aged $18-25$ years $\left(\mathrm{M}_{\mathrm{age}}=21.8\right.$ years $)$ who identified as white $(n=10)$ and white/Native American $(n=1)$. As active exercisers, they attended their current fitness center for an average of 1.74 years, exercising 4-7 days per week ( $M=4.55$ days/week). All the participants were former competitive athletes who transitioned into exercising in fitness center environments after sport. Two women reported continued participation in competitive sport during the study. Participants were purposively sampled with the following eligibility criteria: exercise a minimum of three days per week at a fitness center, engage primarily in individual-based exercise (e.g., weight training, cardiovascular), and possess a cellular smartphone with photography capabilities to be eligible for this study. Once participants were screened for eligibility, maximum variation sampling (Patton, 2015) was employed in an attempt to select a range of fitness centers. Maximum variation sampling allows for the ability to capture, in depth, diversity across a sample while identifying commonalities (e.g., mirrors, locker rooms, messaging) and differences (e.g., culture of the facility) in fitness centers that may play a role in how women experience their body while exercising (Patton, 2015). This resulted in a sample of fitness centers including: college campus recreation centers 
$(n=3)$, commercial chains $(n=3)$, independent studios $(n=2)$, CrossFit $(n=1)$, and employee wellness facilities $(n=2)$ across four states.

This approach to sampling was selected for several reasons. Pietkiewicz and Smith (2014) argue that IPA researchers should aim for a fairly homogeneous sample as the goal of IPA is to describe similarities and differences within a group that has been defined by important, similar variables. Hence, it is recommended that the homogeneity of a sample be determined by the interpretative concerns of the researcher including the degree of similarities or differences to be captured, and pragmatic study design considerations including recruitment. Phenomenological methodology assumes unique experiences exist for each participant based on their subjective engagement and interpretation of their worldview (Pietkiewicz \& Smith, 2014). Therefore, many phenomenological scholars advise against specifying a sample size prior to the commencement of the study. However, Cohen, Kahn, and Steeves (2000) suggest a sample size of less than 10 participants if researchers intend to follow an intensive and interpretive approach to data collection and analysis. Moreover, it is suggested that sample sizes within phenomenological analysis should depend on several key factors including: (1) the depth of the analysis for each case, (2) the richness of the individual cases, (3) how the researcher wants to compare across cases, and (4) any logistical constraints to data collection (Pietkiewicz, \& Smith, 2014). As Pietkiewicz and Smith (2014) state, "IPA researchers should concentrate more on the depth, rather than the breadth, of the study" (p. 9). Together, maximum variation and purposive sampling methods will allow for the elicitation of information-rich cases while accounting for a variation in exercise facilities with hopes of describing the essence of women's exercising body in the broader context of fitness centers. 


\section{Recruitment Flyer}

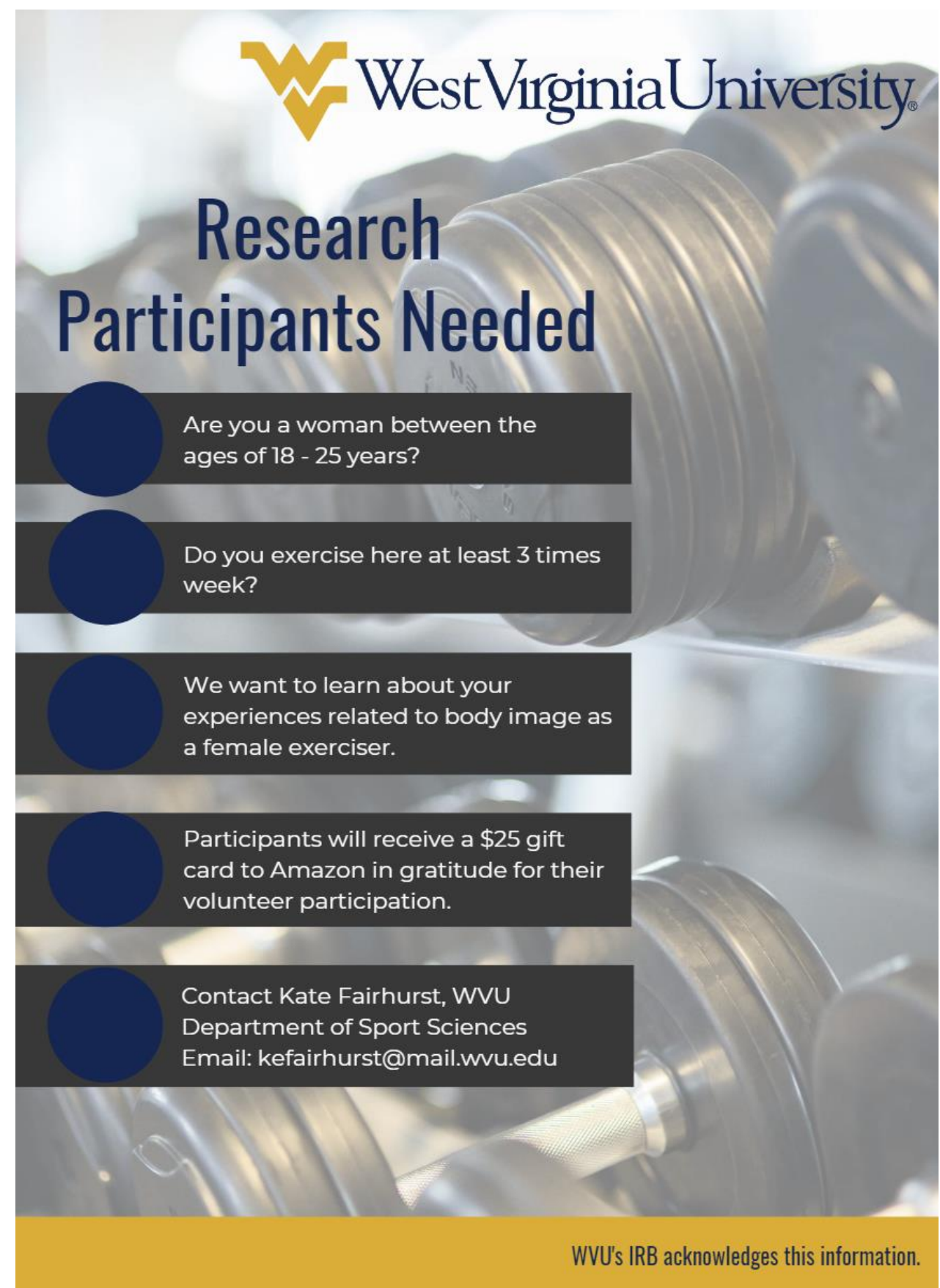




\section{Cover Letter}

\section{WestVirginiaUniversity.}

Dear Participant,

You are being invited to participate in a research study conducted by Katherine E. Fairhurst. M.A. and Dana K. Voelker, PhD in the Department of Sport Sciences from West Virginia University. The purpose of the study is to explore young women's experiences of their body weight, shape, size and appearance while exercising in fitness centers. This research will be used to inform how we create exercise environments that empower young women to develop, and maintain, a healthy relationship with their body and exercise.

The current study will consist of two phases: (1) a two-week photography period, and (2) photoelicited, open-ended interviews via Skype ${ }^{\mathrm{TM}}$. First, you will be asked to complete an initial phone interview lasting 20-30 minutes to collect demographic information and receive instructions for the photography portion of the study. Second, you will be asked to take photographs over a twoweek period with the camera imbedded in your cellular smartphone of factors in your fitness center that are encouraging (e.g., inspiring, vitalizing, giving confidence) and discouraging (e.g., demoralizing, disheartening) to how you feel and think about your body while exercising. Third, you will be asked to participate in a 60-90-minute audio-recorded interview via Skype ${ }^{\mathrm{TM}}$. All interviews will be audio-recorded and transcribed (i.e., a fully type-written copy of the audiorecording).

Please note that your participation is voluntary and that you may refuse to answer any question, decide at any point during the project to discontinue all participation, and choose not to have your data used in any form. The WVU Institutional Review Board's acknowledgement of this study is on file. Your involvement in this project will be kept as confidential as legally possible. If any publication results from this research, all data will be reported in aggregate, and you will not be identified by name. Any hard copy data related to this project will be kept in a locked filing cabinet. Any electronic data related to this project will be kept on password protected computers. All data will be destroyed (i.e., shredded, deleted) following project completion.

As a participant, you may benefit from the reflective nature of the interview and use it as an opportunity to self-reflect on your personal experiences. We anticipate this research project will pose little if no risk to you other than the time it takes to complete the study. However, should you find any of the questions to be considered sensitive, local community resources may be utilized. General resources to assist with these concerns are provided. As a modest compensation for your time and effort, you will receive a $\$ 25$ electronic gift card to Amazon.com following the completion of the study. 
If you have any questions, please contact:

Co-Investigator: Katherine E. Fairhurst, M.A.

WVU College of Physical Activity and Sport Sciences Health \& Education Building, CPASS 241

P.O. Box 6116, 375 Birch Street, Morgantown, WV 26506-6116

kefairhurst@mail.wvu.edu, (681) 668-8069

Principle Investigator: Dana K. Voelker, $\mathrm{PhD}$

WVU College of Physical Activity and Sport Sciences Health \& Education Building, CPASS 207

P.O. Box 6116, 375 Birch Street, Morgantown, WV 26506-6116

dkvoelker@mail.wvu.edu, (304) 293-0871

National Suicide Prevention Lifeline

1-800-273-8255; http://www.suicidepreventionlifeline.org/

National Eating Disorders Association Helpline

1-800-937-2237; http://www.nationaleatingdisorders.org/find-help-support 


\section{Initial Phone Interview}

\section{Demographic Information}

1. Age:

2. Race/ethnicity

3. Highest level of education:

4. Please list any current occupation(s)

5. Name of fitness center that you attend:

\section{Background and Pre-Understanding Questions}

6. Briefly describe the features of your fitness center:

7. What motivated you to begin exercising in a fitness center?

8. Describe current exercise behaviors:

9. How long have you been exercising at your fitness center in total?

10. On average, how many times per week have you done each of the following exercise types:

- Group exercise classes 
- Weight training

- Cardiovascular training

- Exercise at home/outside

\section{Photography Instructions}

"For the next two-weeks, using the camera embedded in your smartphone, take photographs of elements in the exercise facility that are encouraging and discouraging to how you feel and think about your body while exercising. Encouraging can be defined as someone or something that gives you confidence or support, something positive that gives you hope for the future. Similar words to encouraging aspects may be factors that are uplifting, inspiring, or vitalizing to how you feel about your body while exercising in your fitness center. Likewise, discouraging can be defined as factors that cause you to lose confidence or enthusiasm for your body. Similar words to discouraging aspects may be factors that are disheartening or demoralizing to how you feel about your body while exercising in your fitness center. I will ask that, to protect the identity of those in your fitness center, that you avoid taking photographs of people's faces and/or other features that may be identifying.

In addition to the photographs, you will be asked to use the 'log book' provided via email to document additional notes or insights you had during the experience that may not be captured by the photographs you took. Lastly, throughout the two weeks, I will be sending phone or text reminders based on your preference. Once the two-week photography period is complete, please contact Kate Fairhurst (kefairhurst@mail.wvu.edu) to request instructions for sending your collection of photographs electronically. At that time, you will be provided with permission and password protected access to an electronic folder in which you can upload your photographs. Once the photographs have been uploaded, we will schedule a 60-90 minute Skype ${ }^{\mathrm{TM}}$ interview, 
during which we discuss some photographs and your experience with the project. At any time during the photography period, you may contact me at 681.668 .8069 or

kefairhurst@mail.wvu.edu with questions, concerns, or clarifications. After our phone call, I will email you a handout outlining the instructions for your reference."

11. Do you have any questions about the photography instructions?

12. Any other questions or concerns at this time? 


\section{WestVirginiaUniversity.}

\section{Participant Photography Instructions Handout}

\section{Participant Photography Instructions}

$>$ For the next two-weeks, using the camera embedded in your smartphone, take photographs of elements in the exercise facility that are encouraging and discouraging to how you feel and think about your body while exercising.

$\circ$ Encouraging can be defined as someone or something that gives you confidence or support, something positive that gives you hope for the future (similar words may be factors that are uplifting, inspiring, or vitalizing) to how you feel about your body while exercising in your fitness center.

- Discouraging can be defined as factors that cause you to lose confidence or enthusiasm for your body (similar words may be factors that are disheartening or demoralizing) to how you feel about your body while exercising in your fitness center.

Note: To protect the identity of those in your fitness center, please avoid taking photographs of people's faces and/or other features that may be identifying.

$>$ In addition to the photographs, you will be asked to use the 'log book' provided via email to document additional notes or insights you had during the experience that may not be captured by the photographs you took.

$>$ Throughout the two weeks, I will be sending phone or text reminders based on your preference.

$>$ Once the two-week photography period is complete, please contact Kate Fairhurst (kefairhurst@mail.wvu.edu) to request instructions for sending your collection of photographs electronically. At that time, you will be provided with permission and password protected access to an electronic folder in which you can upload your photographs.

$>$ Once the photographs have been uploaded, we will schedule a 60-90 minute Skype ${ }^{\mathrm{TM}}$ interview, during which we discuss some photographs and your experience with the project.

At any time during the photography period, you may contact me at 681.668 .8069 or kefairhurst@mail.wvu.edu with questions, concerns, or clarifications. 
Photography Log Book

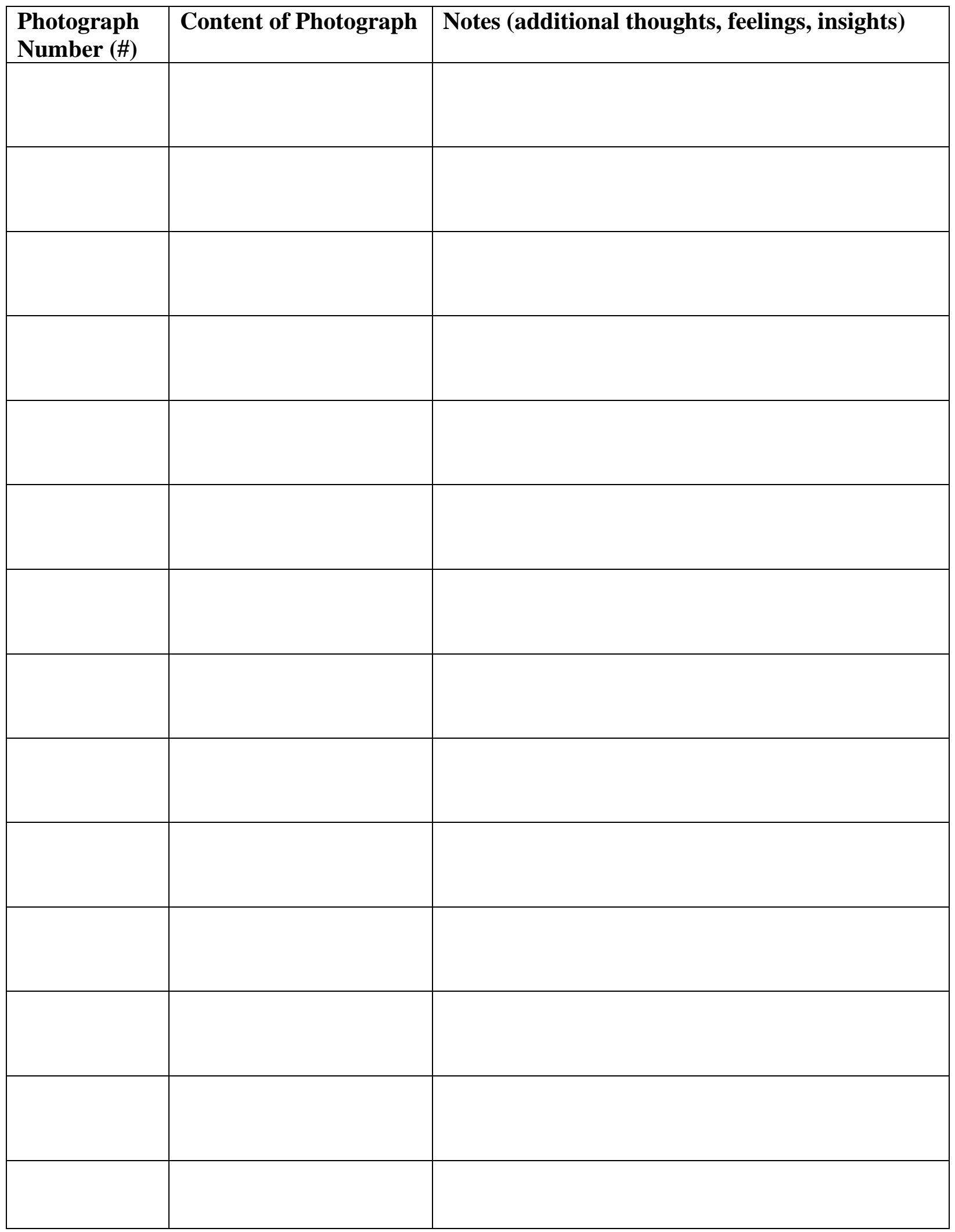




\section{Photo Retrieval Process}

Congratulations on completing Phase 1 of the study! We very much appreciate your ongoing participation and hope that the study has been a valuable experience thus far. At this point, it is time to transfer your collection of photographs and log book to Kate and schedule Phase 2, the interview. This document includes detailed instructions on how to upload your photographs to a password-protected, secured file sharing system.

Look for an email from "FileLocker" which is a password-protected, secured file sharing storage system. The email should look like this:

This file can be uploaded by visiting the URL below:

https://filelocker.wvu.edu/public upload?ticketld=90d2329afe68acdbe0d562b93dedcf11

The requestor also attached this personal message to the file request:

"Here is where you can upload the collection of photographs you took"

Once on the FileLocker website, you should see a screen that looks like this:

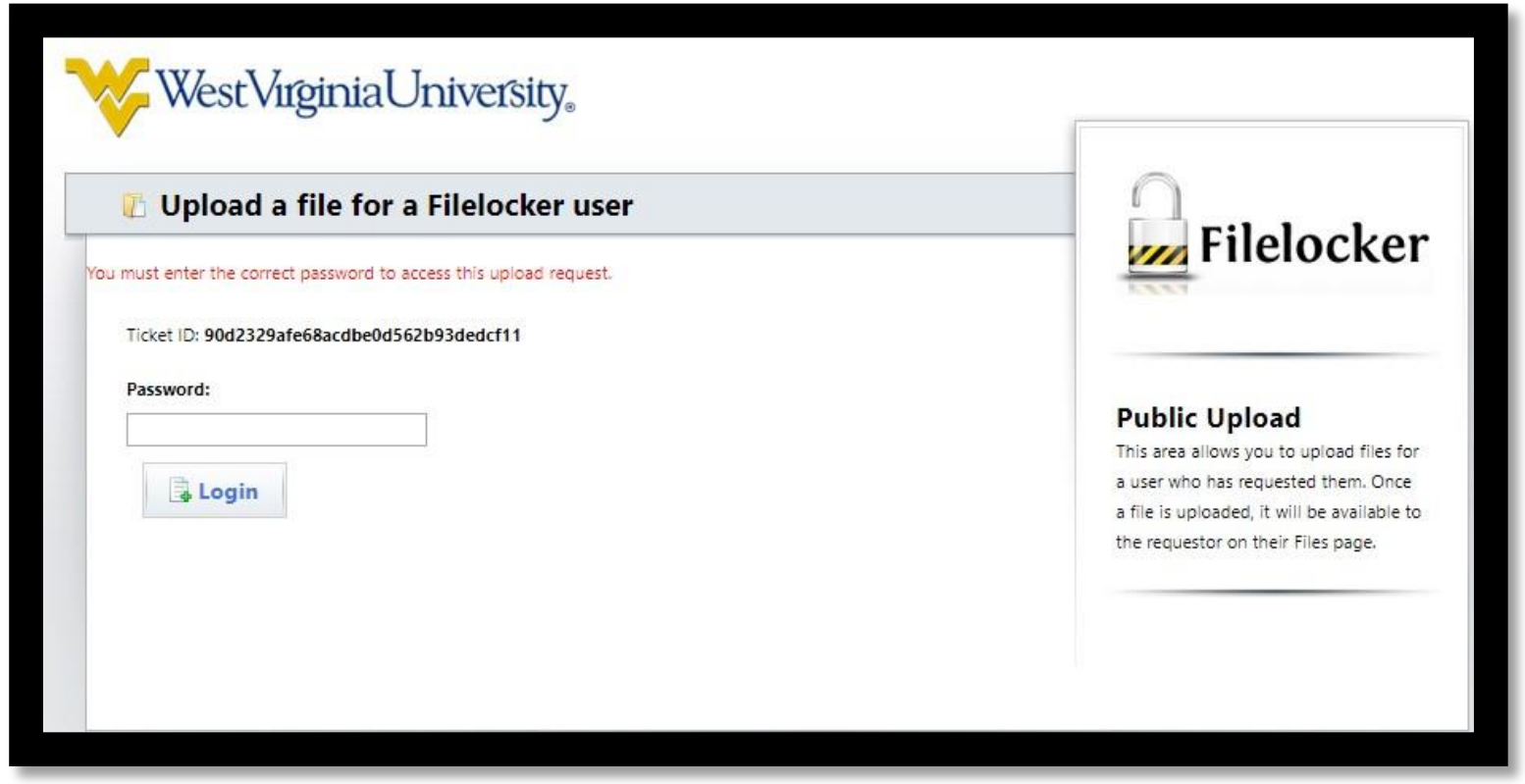

You will need to enter a password to access the file upload area: FitnessCenter 
Once you enter the above password, the next screen is where you can upload the photographs you took. You can upload several photos at once. It is suggested that you upload the photos from your computer for better resolution, upload speeds, and userability.

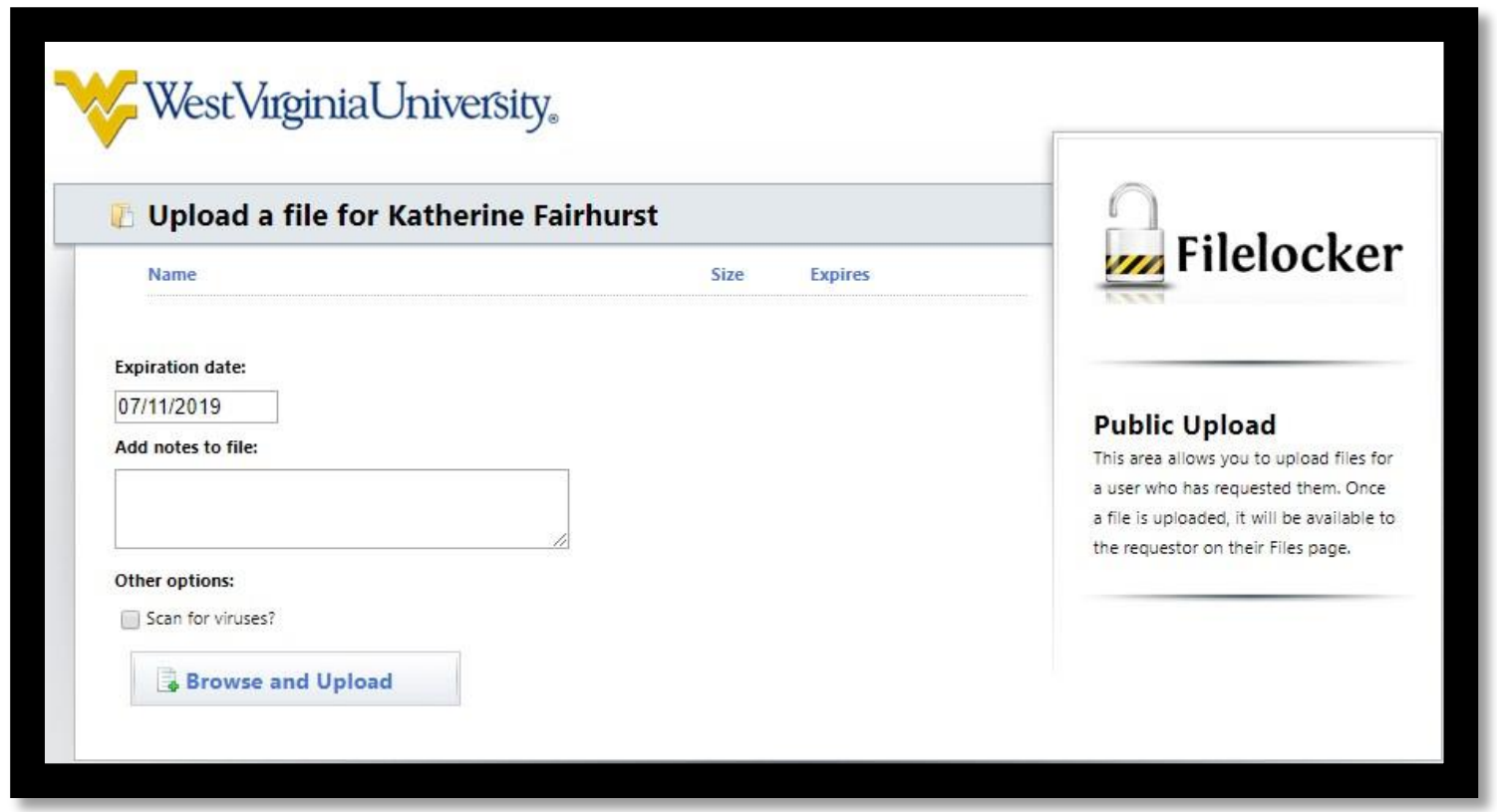

Successfully uploaded files will appear on this screen and look like this:

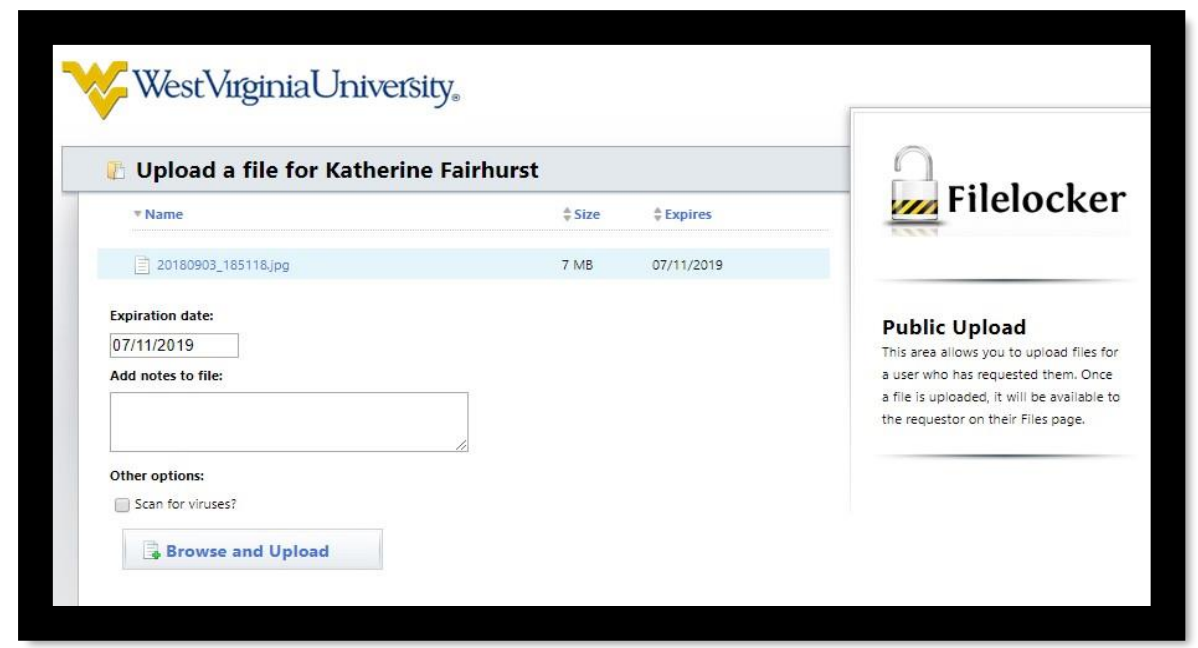

Once you have uploaded the photographs and log book, please contact Kate Fairhurst via email (kefairhurst@mix.wvu.edu) or text (681.668.8069) to notify her that you are ready to schedule Phase 2 of the study! 


\section{Skype $^{\text {TM Interview Guide }}$}

\section{Introduction: Rapport Building}

\section{Background}

- What are your motivations for exercising?

- How would you describe how you feel about exercise?

- How do you feel about your body?

- How is your exercise behavior and physical fitness perceived within your support system and social group?

- If you could use three words to describe how exercise in general makes you feel in your own skin, what would they be? Why?

\section{Pre-understandings}

- What is your perception of the 'fitness culture' in general?

- Describe the features of your fitness center.
○ Amenities
○ Patrons
$\circ$ Your use of the facility

- What would you say the culture of your gym is like (e.g., intensity, goals)?

- If you could use three words to describe how exercising in a gym specifically makes you feel in your skin, what would they be? Why?

\section{Photovoice: SHOWeD questions}

1. Please select a photograph you would like to discuss.

a. You titled this photo What inspired this title?

(1) What do you See here?

(2) What is really Happening here? 
(3) How does this relate to Our lives?

(4) Why does this situation, concern, or strength exist?

(5) What can we Do about it?

**Process to be repeated with several photos until participant signals completion of relevant photos**

2. Please select the 2 most meaningful photographs from your selection and share your reasons for selecting those photographs.

\section{Follow-up Questions}

- $\quad *$ Refer to $\log$ book* What were your notes or insights that you logged that were not captured with the photographs we already discussed?

- What other factors, that were not captured in the photographs, are encouraging to your body image while exercising? Discouraging?

- What was your experience of engaging in the photography portion of the study?

- How has your involvement in this study impacted the way you perceive your body while exercising?

- Reflecting on this experience, what meaning does exercising in a fitness center give your body?

- How has your involvement in this study impacted your views about the female body in the fitness culture in general?

- Do you have any additional comments, insights, or questions you would like to add? 


\section{Appendix D: Case Summaries}

\section{Case Summary: P1 (Sam)}

Background and Demographics

Age: 25

Type of fitness center: "Physical Activity Promotion for All"

\section{The Setting Through Their Eyes}

The broader fitness culture:

- $\quad$ society has made gyms think they need to offer (meaning that fitness centers are simply communicating the ideals from broader culture - perpetuating)

- Dominated by social media models and/or Olympic athletes - the extremes of one of the other - little representation of those in the middle

- The thin ideal is a narrow definition of fit, few women represent it!

Past gym experience:

- Intimidating (dim, heavy weight-lifting, extreme dungeon style gym)

- Large space, crowded, lighting, layout was intimidating

- Male-dominated environment was intimidating (college campus rec center)

- Male-dominated, revealing apparel, females were the minority = intimidated.

Current fitness center:

- Small, diversity in patrons but perceives similarity to herself - was not intimidated

- Management staff that represent an attainable level of fitness encourages sense of belonging

- Perceives center to be a "physical activity promotion for all"

Culture of fitness center:

- Find your own fitness

- Opportunities to learn and perceives support to try

- Encourages comfort and community (relatedness and belonging)

- Belief that fitness center would embrace different ideals but further perpetuates same ideal (confused about how a center could somehow be communicating both)

The Fitness Center Experience

"This is a man's reality"

"It's like they're afraid of strong women"

Example communicating this is the lack of female images on weight machines:

- Questions why women aren't imaged on lower body machines at least

- Creates fear that weightlifting will make women look masculine 
- Communicates that weights are for men

- Representation of thin ideal in fitness center increases self-doubt towards body questions efforts to be healthy and fit

- $\quad$ Surprised by presence of feminine products provided in the restrooms

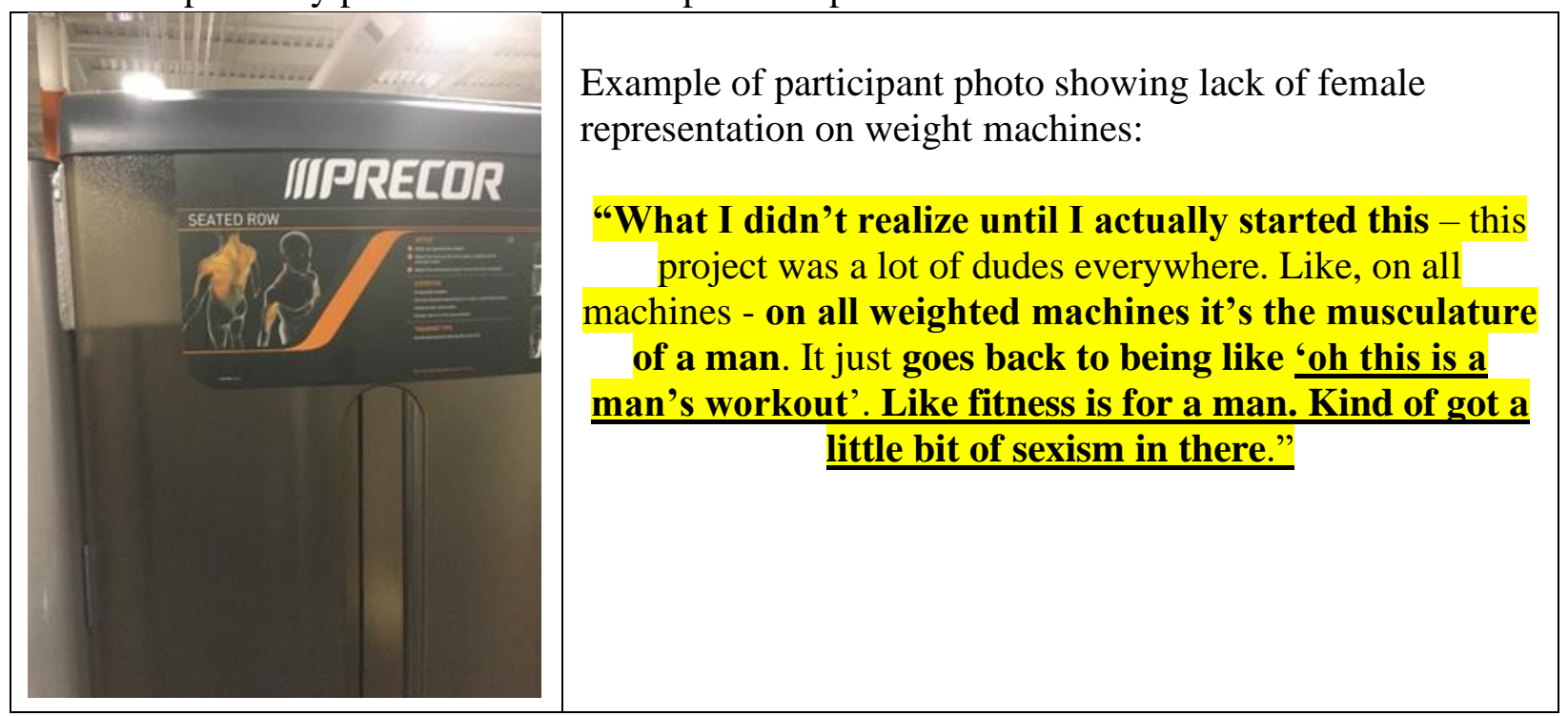

"You need this machine to get thin"

Example of this is the imaging of thin women (no men or average women) in cardio machine screens. Perpetuates the thin ideal and gendered messaging about female-appropriate exercise modalities:

- Perpetuates message that women should do cardio and look a certain way

- Perpetuates traditional gender stereotypes for exercise

- Women belong on cardio machines

- Not representative of muscular, bigger women which is discouraging

- Communicates a reminder that she is not the ideal 


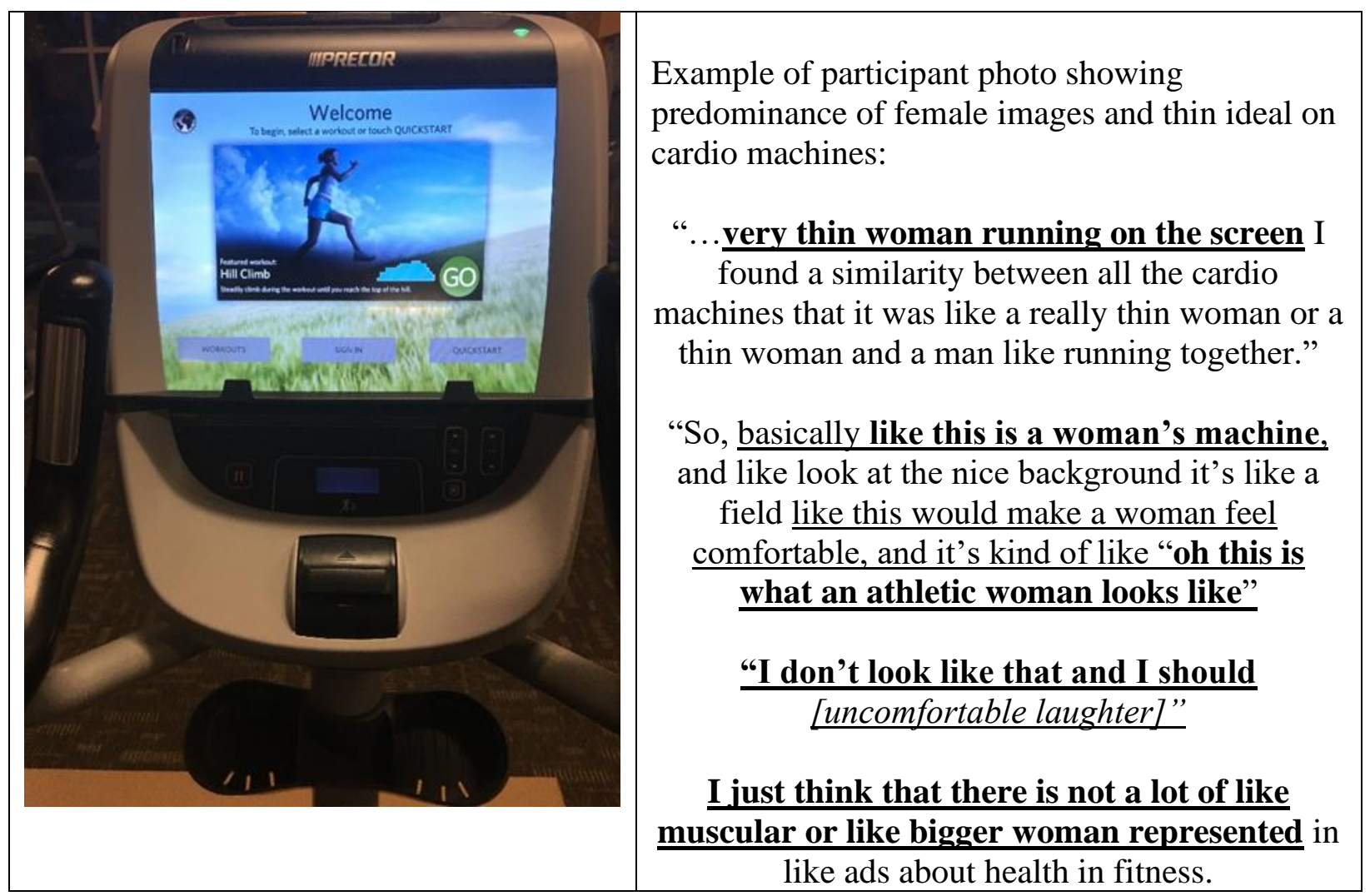

"Mirrors are the worst place to go"

- When struggling with negative body, image mirrors increase awareness of perceived flaws

- Represent reality of one's body, self-comparison to ideal, desire to be tiny - dissonance

- Influences workout length if experienced as negative, which creates avoidance behaviors

Messaging can Buffer a Negative Experience

- Language based messaging that promotes body diversity and body acceptance can help buffer the above experiences

- Decal of encouragement on bathroom mirror encourages self-appreciation

- Self-compassion-based language inspires self-acceptance

- Messaging focused on effort communicates encouragement to try

- Important that these messages do not have images of people on them which typically results in self-comparison 


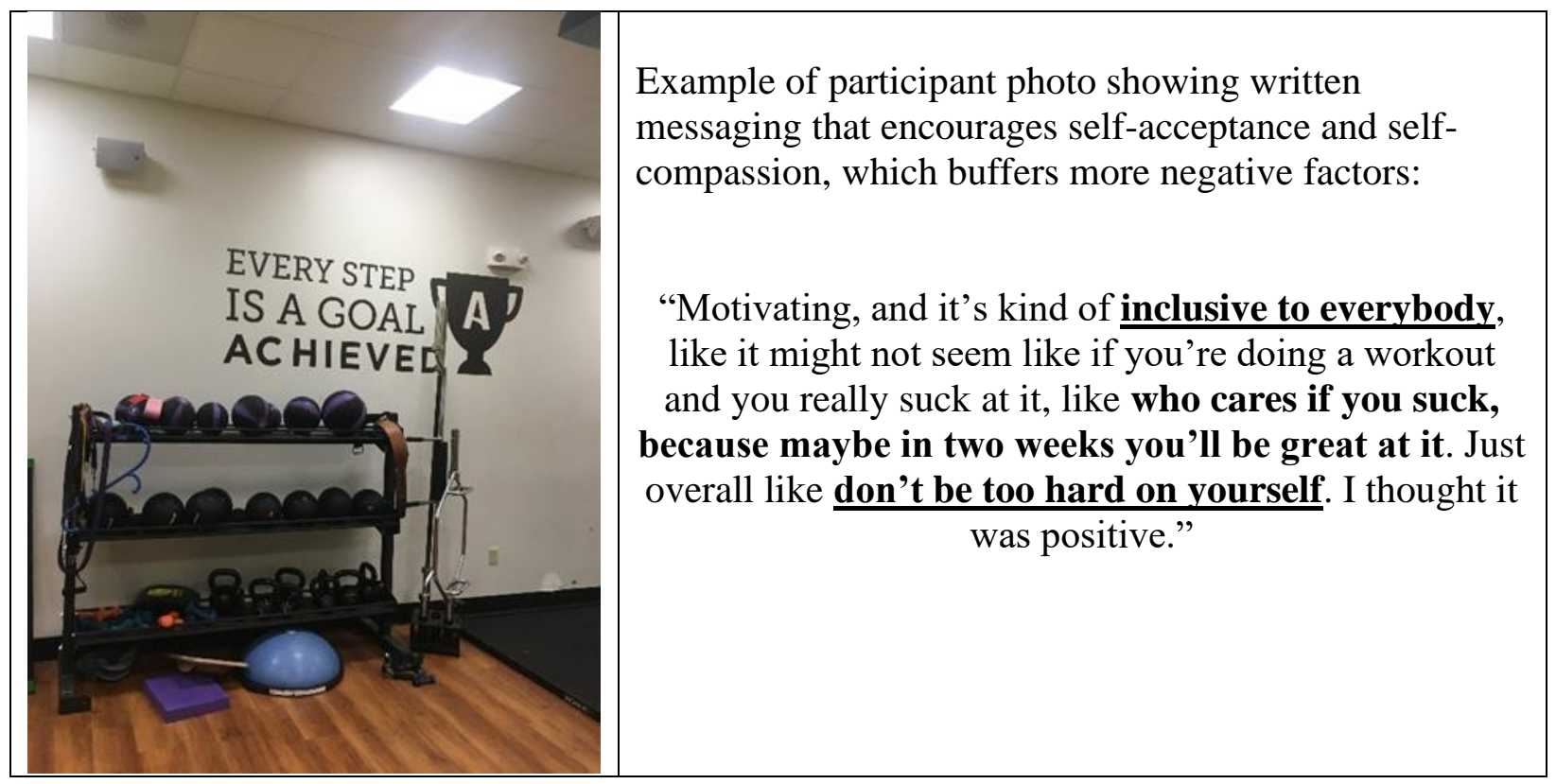

Navigating Their Place

Cautious to Enter the Fitness Center Environment:

- Eased into weight training with help of an ally

- Weight training in open space was uncomfortable

- Started with group classes

- Self-doubt as a beginner, competence was low

- Stuck to cardio machines, controlled, familiar, easy to use, safe space

Relationship with Body:

Body Conflict experience results from perceiving the thin ideal from the broader culture and fitness center environment. Body conflict experienced in several different ways:

- Has internalized that men are to be built up and women are supposed to be hippy

- Enjoys weightlifting but self-conscious about the aesthetic she develops from weights deviant of societal ideal

- Fit ideal of being skinny and muscular is also unrealistic - desire for both, but they are often opposing ideals

- Enjoys the feeling of, and result of, strength but doesn't like "being big"

The experience of conflict with her body shapes how she defines her self-concept:

- She perceives that she is "never going to be small" and explains "bones won't let me"

- Describes self in relation to the ideal. E.g., "genetically big"

- Seems forced to accept a "less than ideal" mentality

The experience of body conflict also seems to shape her exercise behaviors:

1. The scale: use of the fitness center scale depends on how she is training. For example, she "loves" the scale when she is doing cardio training because she can predict that the 
number will do down; she equates a lower number to be "good". However, she "avoids" the scale when weight training because she assumes that an increasing number on the scale is "bad."

2. Choosing workouts: how she exercises is determined by how she perceives her body in relation to the ideal. For example, if she feels she "has bulked up in her upper body," she will increase her cardio to reduce size and get closer to the ideal. The internalized thin ideal seems to have more power over her behavior than the experiences of strength, power, and positive feelings associated with exercise.

\section{Reflections on the PhotoVoice Experience}

Engaging in the research project itself provided an opportunity to build awareness of the fitness center environment in several ways:

- Awareness of gendered environment and sexist messaging

- What, how, and why the fitness center offers to women, both positive and negative

- Awareness of perceived pressure to behave in ways that one isn't comfortable with to conform

- Regardless of the fitness center environment's influence over the body experience, selfacceptance is a process, not by choice, but by necessity because much of what fitness center's offer is out of one's control

\section{Case Summary: P2 (Lex)}

Background and Demographics

Age: 23

Type of fitness center: "Physical Activity Promotion for All"

The Setting Through Their Eyes

The Broader Fitness Culture:

- Appearance focused - social media and boom of athletic apparel brands

- Fitness apparel brands create a focus on looks; creates an assumption that women who wear clothes from social media brands only exercise to fit the social media ideal

- Because of the culture, there are more women exercising for disingenuous motives - to look like a fitness person, rather than be a fitness person (for social media)

- Certain body features are "trendy," which changes very quickly - hard to keep up with

- Social media suggests that one must be out of the ordinary to be worthy of posting pictures (regular people don't have anything 'worth' posting)

- Can be Informative - social media provides instant access to knowledge and instruction

How she chose her fitness center:

- Availability and geography were important factors

- Current facility is body friendly 
- Current facility enforces a dress code which encourages patrons to dress for comfort and de-emphasizes the appearance of the body

- Past experience in fitness centers with revealing attire triggered negative bodycomparison experience

Describing the physical space of current fitness center:

- Balcony overlooking lower fitness

- Two separate floors - weights downstairs, cardio upstairs

- Bright lightening, natural light with windows

- Cycle studio Set-up: mirrorless, disco ball, dark lighting, motivational quotes directed at effort

- In cycle classes, instructor gives participants control over their effort and encourages them to push their own limits

- Dress code poster enforced modest attire

- Fake trees throughout the gym

- Locker rooms

- Scale directly at front of the entrance "numbers matter"

- Mirrors in front of free weight area and machines

- Neutral music

- NO mirrors in cardio area

- Posters with programs, group exercise classes, small group training, "women and weights" introduction program

- Social media pages advertising group exercise classes, and "fit your fit" initiative

- Imaging at current fitness center feature "normal" people

\section{The Fitness Experience}

"[I'm] ten times more confident because I won't see that"

Intention creation, and enforcement, of a dress code discourages the presence of the types of apparel that trigger a negative body comparison process. The dress code encourages modest attire, redirects attention from appearance, reduces body comparison, and the accepted attire aligns with her personal comfort level. 


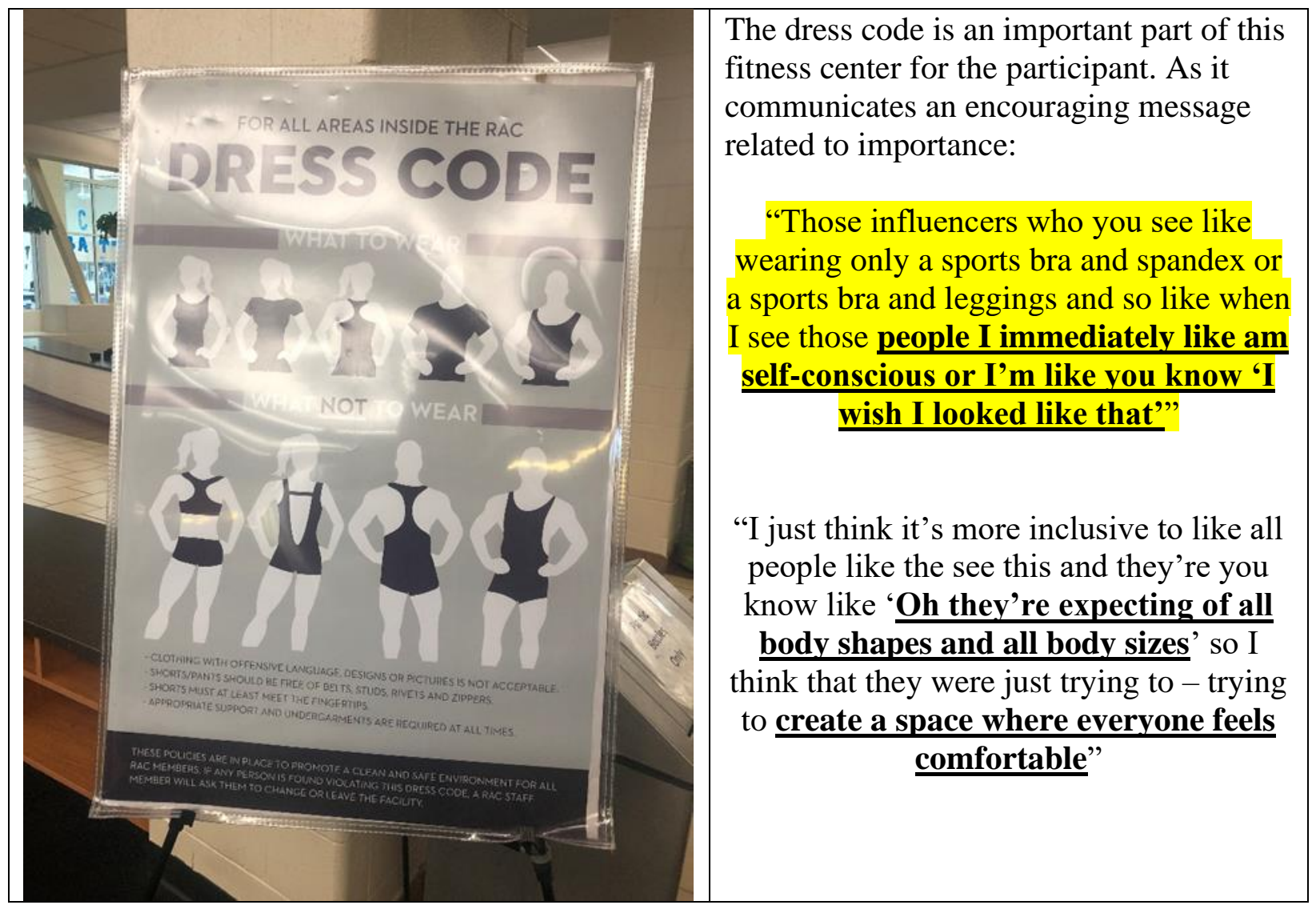

Programs for women that reject gender norms of fitness culture:

- Weight training group for women, offered in different area of the gym creates safe space

- Even though this participant hasn't taken the class, it's comforting for her to know that women doing weights is accepted and promoted at her facility

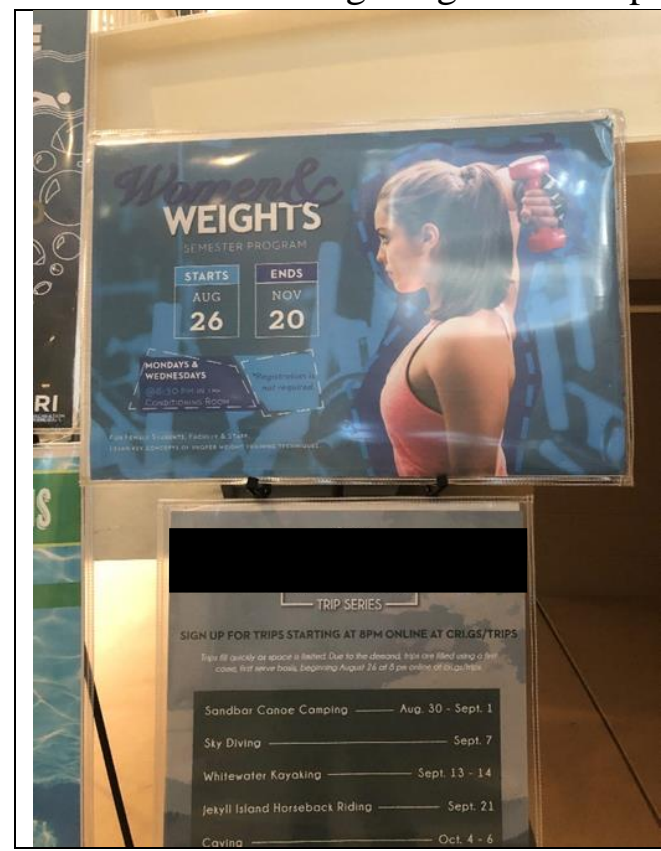

Programs, like "Women and Weights", normalize weight training for women. Embedded programs that reject gender stereotypes, encourage women to feel comfortable trying something new:

"So, I took a picture of this because I just think again this is a pretty unique program. I guess I just never heard of a program like this at [past fitness center] and so I think that this is pretty cool to have like a women or female specific class. Because of maybe people having body image issues or you know concerns about working out in front of other people, this gives them a safe space to do that" 
"I'm going to look at myself and the fold"

Appreciative of areas of the gym without mirrors, specifically in areas where she would do core exercises:

- Core exercise require socially unacceptable fat folds around your middle

- Mirrors in the area bring that flaw to awareness. This gym does not have mirrors there, empowering her to do core exercises regardless

- Some exercises are not "flattering" by society's standards of how women should look

- Mirrors in these areas are discouraging and create avoidance

Navigating Their Place

Body Duality: Fitness is an important part of her identity. She describes her self-concept in relation to the two ideals, broader society's thin ideal and the fit culture's muscular ideal:

- "In-between" the ideals highlighted in the media and social media

- "Taller and bigger than everyone"

- Taller, bigger boobs, not small features

- Low self-confidence to wear form fitting, revealing workout attire

Body duality and cognitive dissonance is further experienced when she:

- Feels strong at current gym because the environment encourages adjusting workouts, embracing failure, and trying to push yourself

- BUT Feels self-conscious at current gym because she perceives her physical features outside the norm

- Lastly, self-conscious when focused on appearance, but appreciative when focused on her body's abilities

Reflections of the PhotoVoice Experience

Engaging in the research project itself provided an opportunity to build awareness of herself and her actions in the fitness center environment in several ways:

- Realized that she perceives herself as ordinary and not different enough to take photos of anything worth taking photos of

- Increased awareness of her apparel choices and what she communicates through the attire she wears. Asks herself, "Am I dressing in ways that make people comfortable or uncomfortable?"

- Changed her motivation for why she went to the gym to a more extrinsic motivator (taking photographs)

\section{Case Summary: P3 (Bri)}

Background and Demographics

Age: 22

Type of fitness center: Specialized Studio 
The Setting Through Their Eyes

The Fitness Culture:

- Instagram influencers (false/incorrect information, misleading)

- Message that you have to look extremely fit to be legitimate - all appearance-focused

- Negative culture centered around creating a following - if you don't have a strong following, you aren't worth it

- People are willing to go to extremes to be accepted by negative culture of fitness

- Include unregulated supplement advertisements that perpetuate the ideal

Prior fitness center:

- Male-dominated in weight area (resulted in avoidance because of restrictive environment)

- Sexist environment - few girls "why is girl down here?"

After negative experience at previous fitness center (conventional gym), intentionally sought out a fitness center that embraced positive values:

- Current facility embraces community

- Club mantra aligns with personal values

- Current facility communicates a non-judgmental, accepting environment with the message that personal growth is the common goal

- Premium cost of the gym = perceives higher value in the exercise experience

- Culture supports individuality, personal growth

- Fitness center and instructors are aligned in communicating message "intention is to put best effort forward" regardless of why you are here

- Social media efforts made by this fitness center are focused around feeling healthier, and wellness

- Culture of "We want you here, you are wanted." Big difference from past experiences of judgement.

- Encouragement of core values creates a positive connection with self and others

- Deliberate attempts to promote body-image positive environment

The Physical Space of the Fitness Center:

- No mirrors, no lights

- Dark studio encourages focus on self, individual workouts among a group, effort and process orientation led by the instructor

- Lack of mirrors minimizes self-criticism and encourages focus on internal process

- Challenge board encourages effort-based competition

- Encouraging to have quotes located in areas that typically motivate self-criticism - (E.g., Bathrooms include encouraging messages "hello beautiful")

- Encouraging Factor to have mural with motivational quotes - "just ride" which communicates dedication, hard work, etc.

- Progress wall - congruent with core values of fitness center 


\section{The Fitness Experience}

\section{Comparing Fitness Center Experiences}

Past experiences in conventionally designed fitness centers:

- Fear of judgement from others as new exerciser (difficult to gain entry)

- Messaging - perceived being judged for form or not lifting heavy enough

- Conflicted - wanted to lift weights, but culture not welcoming to women (body conflict)

- "Planet Fitness" attempts to be inclusive, yet she still experiences judgement and social comparison

- Motivated to self-present, gender stereotypes

- Initial exercise behaviors in this setting aligned with societal messaging about women in exercise (cardio + light weights)

Current fitness center: unique, boutique style fitness center designed in contrast to conventional gym:

- Makes her feel confident, happy, strong in her skin

- Messaging uses language to direct energy to present moment, which creates positive body experience

- Mindfulness based language of "rules" encourages positive relationship with exercise

- Mindfulness based language encourages positive mindset 'feeling over fit' promotes wellbeing and comfort in your own skin

- "No need to fight against your body here"

- Promotes message that self-improvement is a process, self-kindness, self-compassion, pursuit of autonomy and self-actualization

- Quotes about character over body-related comments buffers natural self-criticism process and encourages better sense of self

- Progress and achievement in the service of self improves how you feel in your own skin focus shifts away from body to holistic wellbeing

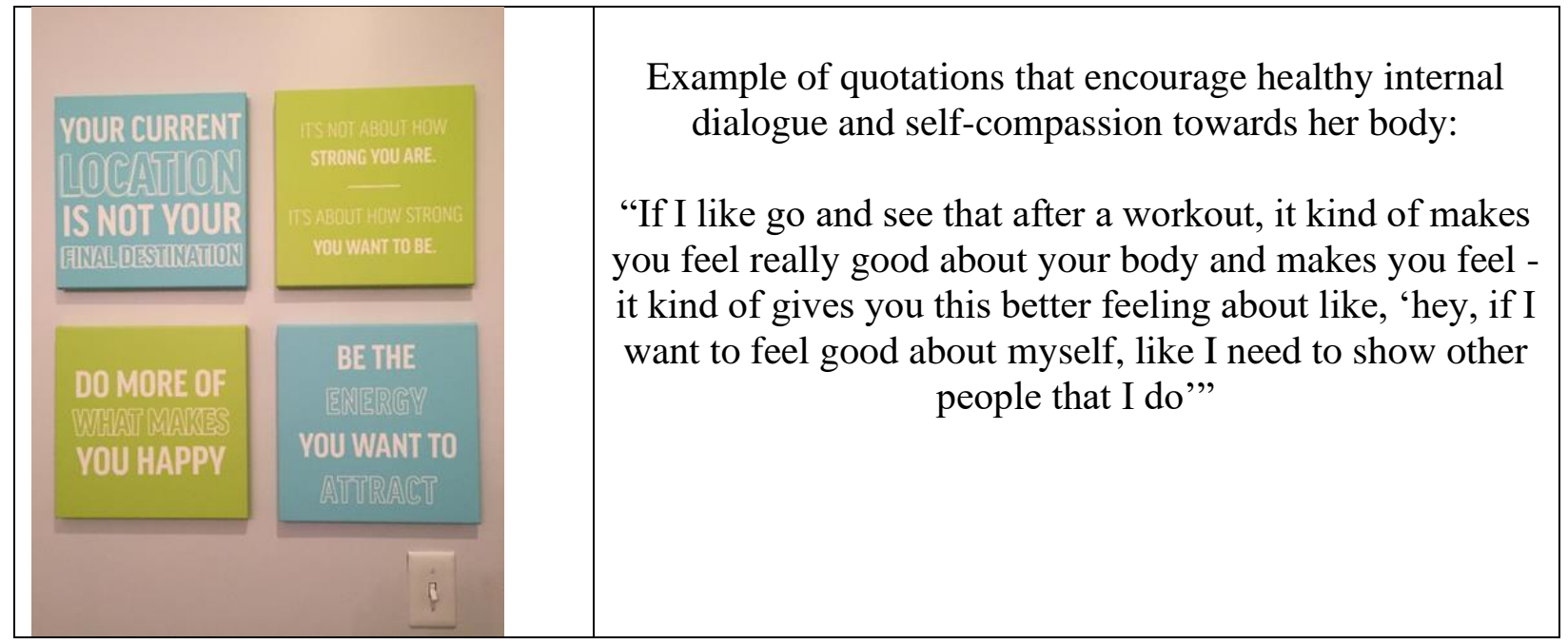




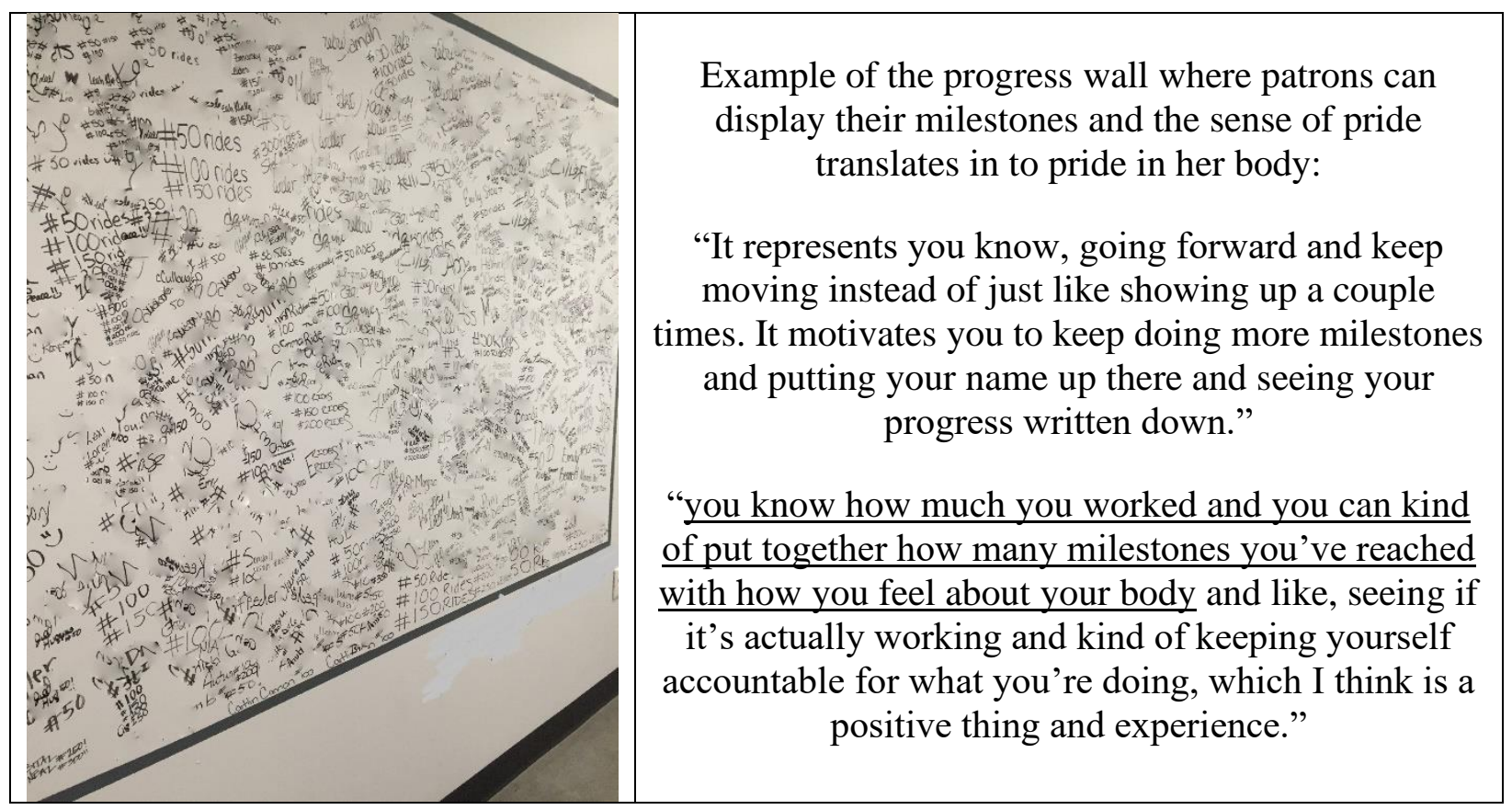

Navigating Their Place

Initial caution toenter fitness center environment:

- Ease into fitness center by starting in a group context with exercise classes

- Transition out of negative experience was aided by peer

- Left discouraging environment after 2-3 years for current center

Relationship with her body:

- Can fall victim to self-doubt and comparison based on fitness culture messaging but attempts to dispute these thoughts; this new setting assists with that

- Describes body image as situation, context, and temporally specific

- She has confidence in her body when she feels she has a healthy mindset towards exercise

- Shift in understanding about what healthy looks like - behaviors, habits, positively oriented action ' body image improvement because you KNOW you're DOING healthy things

\section{Reflections of the PhotoVoice Experience}

Engaging in the research project itself provided an opportunity to build awareness of the fitness center environment in several ways:

- Heightened awareness of self in space, self-comparison, motivation (both positive and negative)

- Difference in body experience between working out in conventional gym versus working out in a dark room

- When exercising in a place congruent with personal identity, that place can become integrated into one's identity 
- Awareness of how environment influences our internal body image experience

- Healthy, positive body image is experienced when internal dialogue, thoughts, feelings, and behaviors are aligned and congruent with personal values towards health and fitness.

- Rejection of societal ideals through interaction with field of study - awareness that skinny does not equal healthy

\section{Case Summary: P4 (Emi)}

Background and Demographics

Age: 20

Type of fitness center: "Functional Fitness" (CrossFit Gym)

The Setting Through Their Eyes

The Fitness Culture:

- Dominated by social media/media that pushes negative, appearance-focused goals and products that promote this goal

- Different fitness subcultures do have different approaches to body diversity

- Subculture that the participant identifies with accepts muscular body types for women

- Belief that variety breeds inclusivity and community breeds understanding, which isn't represented at higher levels of society, but is in her fitness subculture

Choosing a Preferred Fitness Center

- Guided by past experience in traditional gyms. Belief that conventional fitness centers intentionally work to distract you from (what is assumed to be) a negative experience of working out (E.g., putting TVs on cardio machines)

- Sought out fitness modality that was familiar to her gymnastics background with some equipment she felt competent using

Current Fitness Center:

- described as narrow, no mirrors, no posters, CrossFit equipment, nonconventional/familiar equipment, dimly lit, chalk on the ground, messy

Culture of Current Fitness Center:

- Perceives that body diversity is represented, which challenges the higher level societal ideal for women's bodies

- Presence of coach during each session sets the tone that "scaling" workouts to individual ability is respected, recommended, and encouraged

- Strong focus on performance and making progress, which diverts attention away from appearance and focuses more on function

- Culture that highlights and encourages each other on their own fitness path motivates her to improve her performance, but also act as a positive member of the fitness center 


\section{The Fitness Experience}

"Don't feel bad about yourself. Like, it's hard."

Fitness Center Mission Statement: The language that is used in the mission statement provides comfort and self-acceptance where self-criticism would be natural. The culture of selfcompassion/self-acceptance is shown through quotations and messages like the one below. Participant shared that this factor is not directly related to her body but indirectly impacts how she feels in her body by encouraging development of healthy internal dialogue.

\begin{tabular}{|c|c|}
\hline $\begin{array}{l}\text { CRDSSFIT } \\
\text { IT DOESN'T MATTER WHERE YOU FINISH, } \\
\text { ONLY THAT YOU FINISH WITH INTEERITY } \\
\text { AND GIVE IT A SOLID EFFIRT. } \\
\text { LEAVE THE GYM BETTER THAN WHEN } \\
\text { YOU WALKED IN. } \\
\text { HEAD HIGH AND BE PRDUD. } \\
\text { THIS STUFF IS HARD. }\end{array}$ & $\begin{array}{l}\text { Example of participant's inclusion of } \\
\text { messages that focus on effort, self-kindness, } \\
\text { and self-compassion when fitness is difficult: } \\
\text { "I thought that it was a very encouraging } \\
\text { thing to have because even when I do bad - } \\
\text { or I feel like I had a crappy workout or didn't } \\
\text { perform well, at least I went there. - I think } \\
\text { it's aimed more towards your perception } \\
\text { and, more like mental and less body image. } \\
\text { So, I think it's more to help you mentally } \\
\text { push through it. Like it says like "Be proud, } \\
\text { this stuff is hard", like don't feel bad about } \\
\text { yourself. Like, it's hard." }\end{array}$ \\
\hline
\end{tabular}

"You're being guided to success"

- Confidence in a coach can lead to confidence in the exercise which can lead to confidence in self

- Safety and relatedness in an environment of learning inspires self-acceptance

- Past experience in a traditional gym created doubt in exercises and doubt in self as a result; most traditional gyms require one to start out their own (walk in and go exercise) 


Photo shows the whiteboard where the coach
communicates the scaled exercises. This
represents an inclusive and understanding
learning environment:
"On the board, they'll put scaling options.
'Here's the traditional weight, but also here
are some other things that you could do if
you're not comfortable doing that'. I like the
teaching slash learning aspect of it, that
you're being guided to success, like you
weren't just - like I walked into [name of
traditional fitness center] and just kind of did
whatever I wanted to do. There's no guidance
really."

Navigating Their Place

Pushing through Fear:

- Non-conventional equipment may present an intimidating environment - threat to sense of competence - highlights lack of belonging in this context

- With time and exposure, courage to explore transformed into self-confidence and confidence in her skin

Relationship with her Body:

- Confident in her appearance and has a positive body image (thinks she looks "good") because she has maintained the physique she identifies with as an athlete

- Desire to self-present as competent - when threatened, it manifests as self-consciousness in skin

- Experiences some body duality in that the broader culture perceives muscles as too much but within her subculture, it's accepted

\section{Reflections of the PhotoVoice Experience}

Engaging in the research project itself provided an opportunity to build awareness of the positive aspects of her current fitness center (e.g., familiar equipment, chalk on the ground, competitive but community oriented) which assists the development of a positive relationship with her body and exercise.

\section{Case Summary: P5 (Amy)}

Background and Demographics

Age: 21 
Type of fitness center: Appearance-focused, Body Sculpting

The Setting Through Their Eyes

The Fitness Culture: belief that exercise is medicine, but sees the fitness culture prioritizing bodybuilders, aesthetics. She notes that she is not active on social media to avoid negative culture.

How She Chose THIS Fitness Center:

- Equipment and program offerings were more important that the aesthetics

- Sought out features that were positive experiences for her in the past (fitness classes, variety, pool, etc.)

The Physical Space:

- Dominated by mirrors, dimly lit, turf area, concrete floors, functional training zone, traditional weightlifting equipment

- Absence of imaging in the gym - no pictures of people

Culture of Fitness Center: describes that there is comradery among patrons, friendly, motivating but does notice a difference in how men are treated and how women are treated - men judge women for being in the weight area. Fitness centers in general promote body insecurity not present in other life contexts.

\section{The Fitness Experience}

"Favorite thing to see in the gym"

Dumbbell section of the fitness center represents freedom to choose her exercises that help her feel strong and confident in her body, see visible incremental progress in her weights used, and feels empowered to be creative. She spends the majority of her workouts in this area. A similar positive experience occurs when using Smith Machines because one can observe incremental improvements in strength. 


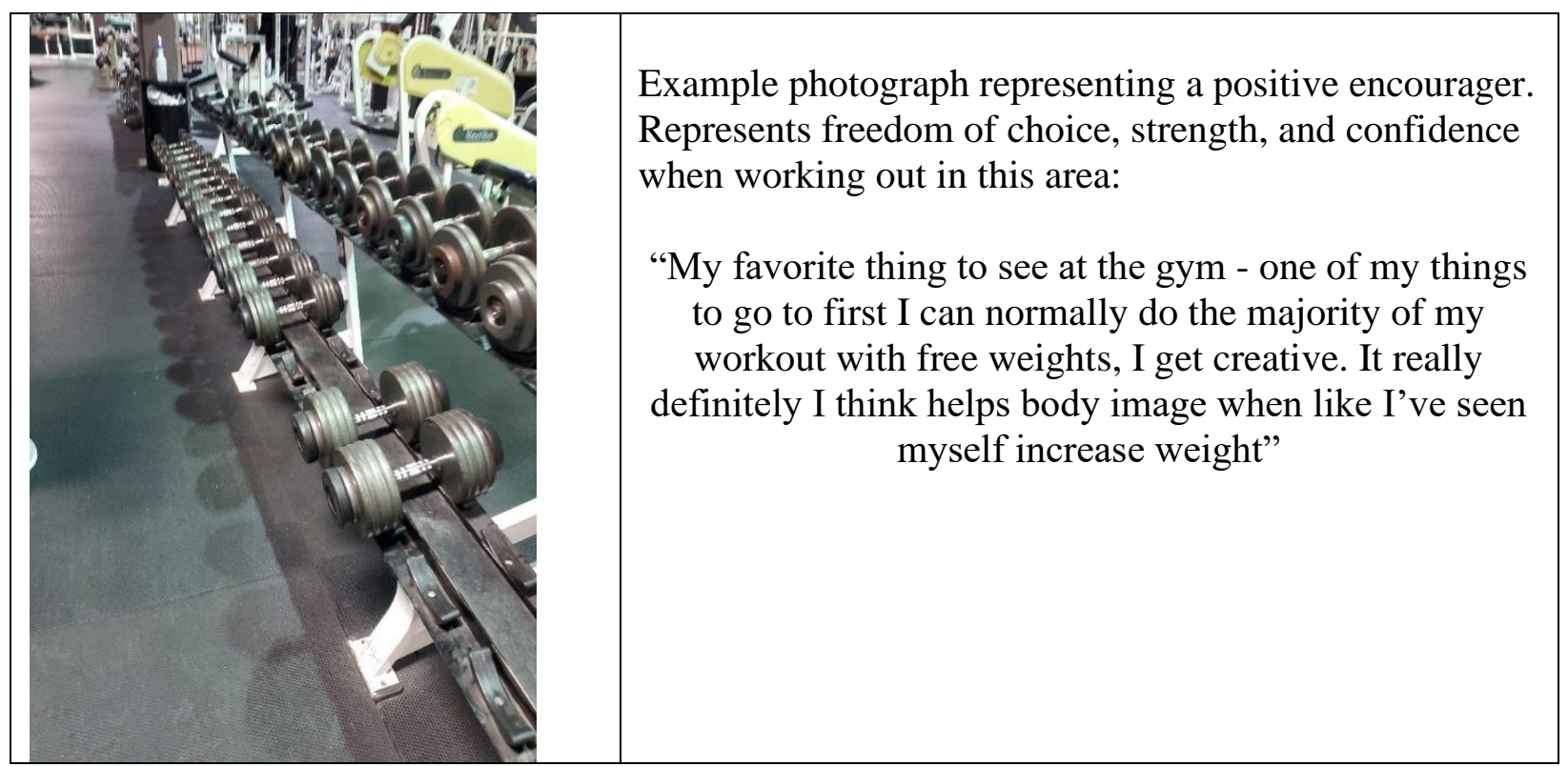

"Being stared at"

Male-dominated weight area and expressed that she is often the only female exercising in their area. This is an open-area which creates a conflicting body experience. On one hand, she is selfconscious of being one of few women (feels objectified as a woman in male-dominated area). On the other hand, she feels proud and strong in her body because she is challenging stereotypes that women don't belong in the weight area (advocating for women in weights).

"There is a lot of men back there like there's
probably at least ten that day and they're all huge
guys like they have mostly like biceps and stuff"
"I went up to the bench beside this one guy and
he just gives me this funny look, like 'what are
you doing back here' and I go grab some weight
and start lifting and he's still just sitting there like
'what are you doing'. That makes me a little
uncomfortable sometimes, like I've had, not
necessarily this instance, but I've had people like
give me that like up down look you know like
Oh I'm checking you out', which I'm like that's
not what I'm here for"

"Around this environment, they become insecurities"

Locker room area represents a negative body experience, a feeling of exposure, fear of judgement by other women, and body comparison experiences. Strong, salient experience which 
promotes avoidance behaviors. Participant highly identifies with exercising for health and wellness; experiences negative emotions towards her body when in an environment which focuses on appearance.

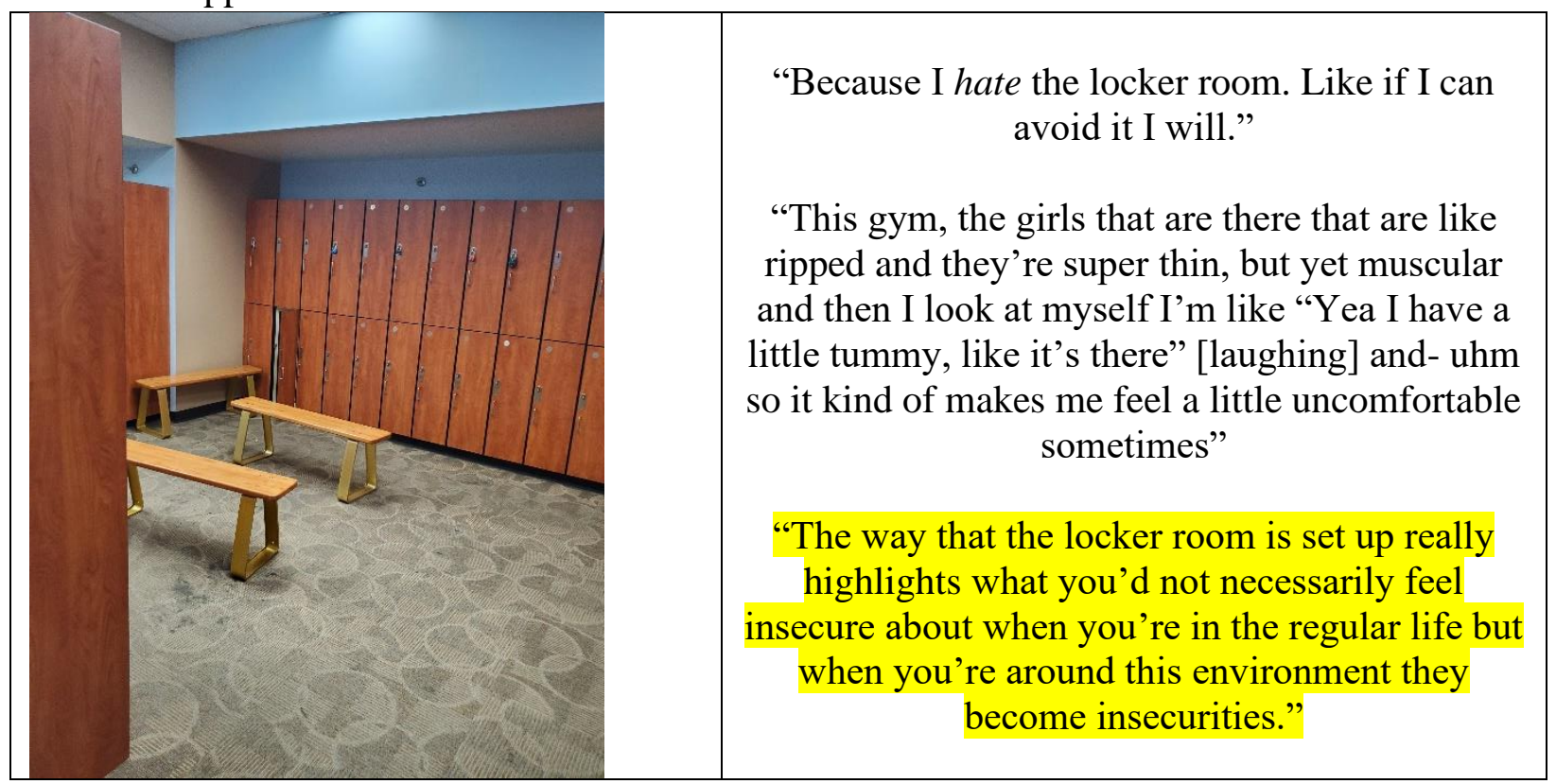

"Catch at a bad angle"

"[Need to] tighten up"

Mirrors around the fitness center create a negative body experience, which creates cognitive dissonance. Discouraged when she "catch[es] [herself] at a bad angle," which triggers inner dialogue such as "I could lose a little weight there," thus impacting her desire to modify exercise behavior by doing cardiovascular training (alignment with gender messages about women doing cardio) to "lose that couple pounds."

Navigating Their Place

Wrestling with Dissonance:

Relationship with Her Body:

"Love/Hate" relationship with her body, which shifts depending on several factors, including: day, time, context. While exercising, she feels fit, strong, and powerful in her skin but can experience self-criticism when comparing herself to societal body ideals or engaging in body comparison with other women in the locker rooms.

Body Conflict: experiences body conflict as she describes self as "curvier" than thin ideal, and has a desire to meet fit ideal (muscular/lean), which may not be what her body is capable of achieving. 
Self-conscious about being self-conscious: assumption that she is the only woman uncomfortable in her skin (particularly when in the locker room) when observing women walking confidently with little clothing on. She questions HOW it's possible for a woman to be body confident.

Avoidance: Modifies exercise behaviors to avoid locker-room area. She avoids sweating during her workouts, to avoid showers/locker rooms, and therefore, avoid negative body experience. She will further modify her workout schedule to avoid locker rooms (E.g., wake up earlier in the morning so she has time to return home to shower)

Being an Advocate:

- Lifting weights in male-dominated environment is empowering; "I belong here"

- Rejects norms/ideals that women are not strong by lifting weights regardless

- Being strong means being capable

- Empowering to reject societal norms for women in exercise

- Acts as an ally to encourage other women to join the weight area (specifically her mom)

Reflections of the PhotoVoice Experience

Engaging in the research project itself provided an opportunity to build awareness of the fitness center environment in several ways:

- Built empathy for how other women may feel, intimidation

- How the environment influences your self-image (makes it worse on a negative body image day)

- Reflect on how the gym impacts her body image personally

- No matter how aware people are of ideals and genetics, they can't help but internalize societal ideals

\section{Case Summary: P6 (Ali)}

\section{Background and Demographics}

Age: 24

Type of fitness center: "Health and Wellness"

\section{The Setting Through Their Eyes}

The fitness culture:

- Represented by aesthetics, an image, and a narrow definition of what it means to be fit

- It fluctuates depending on societal standards for women's activewear (clothing)

- Any educational resources provided perpetuate aesthetic-based motives for exercise

- Fitness culture pressures women to "post" to be defined as fit; "If you didn't post your workout, did you even do it?"

- Sale of products (clothes, supplements, etc.)- image includes clothing labels, must wear to be defined as the right kind of fit 
Describing the Fitness Center:

Freedom of Choice: described the facility as open space with many options for exercise encouraging comfort, enjoyment, and discovery of new ways to experience exercise. Included are non-equipment-based offerings with high-end exercise programs, health and wellness smoothie bar, pro-shop, spa, and pool. Staff was described as providing a welcoming environment by not differentiating themselves as "experts".

The Fitness Center Culture:

- THIS fitness center itself represents the act of "exercise" - her gym or no gym

- Does not encourage any extremes of fitness (i.e., body building, powerlifting, elite athletics, etc.)

- "What fitness is best for you"

- Apparel seen in broader fitness culture is present at this fitness center. Participant identifies with this.

The Fitness Experience

Related to early motivations for fitness, past fitness center experiences included internalizing gender stereotypes related to exercises, strength, and women in the gym. However, in this gym, the assumption that women are weak is challenged when she experiences increases in strength, which increases confidence in self, her body, and her sense of belonging in the gym.

Equipment - Freedom of Choice and Private Sub-Spaces:

- Different weighted free-style equipment provides variety

- Equipment that allows WANT rather than SHOULD type of workouts (autonomy)

- Space that allows for self-expression through exercises of choice

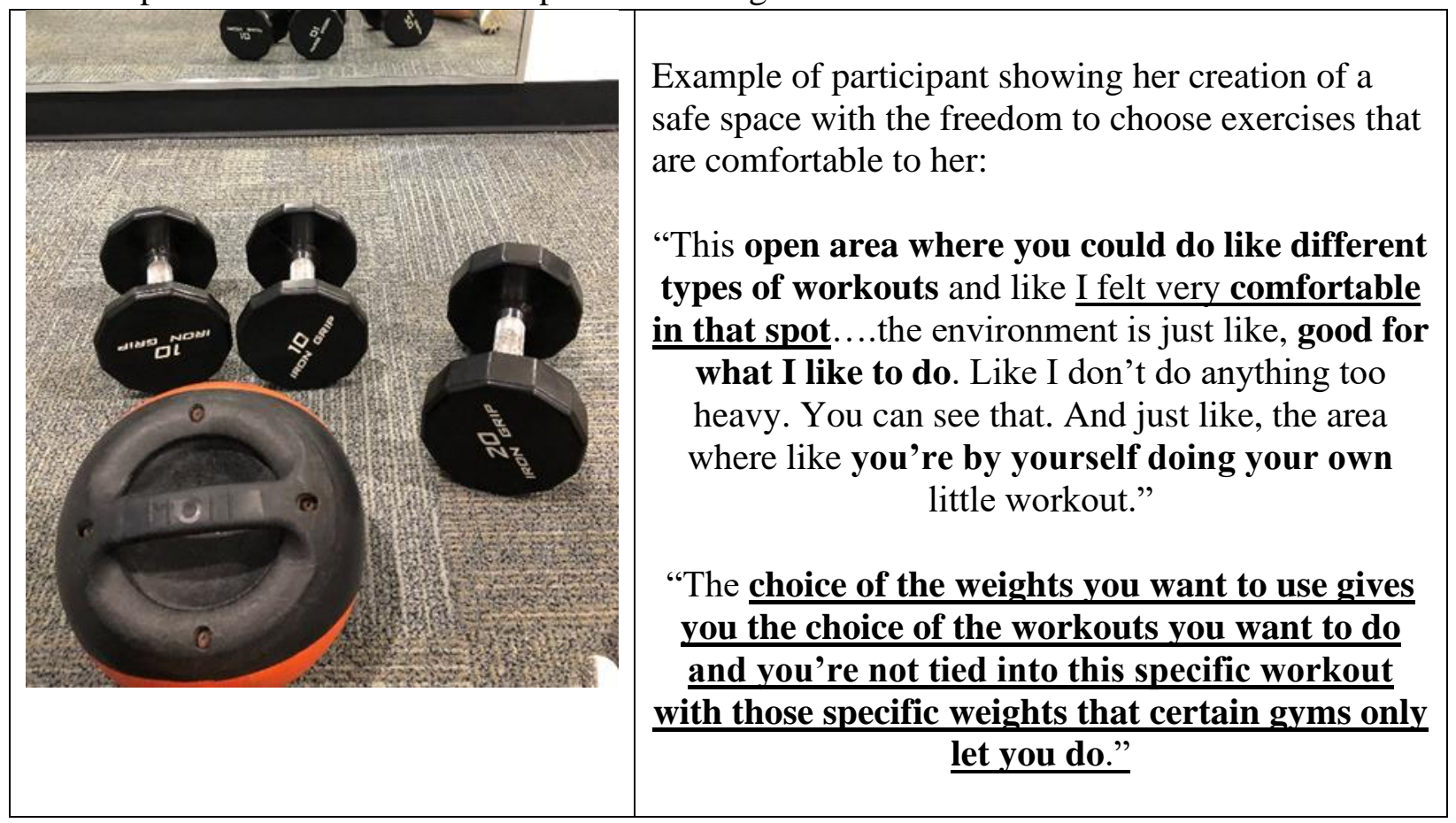


Messaging (Quotations):

- Related to effort, humor, focused on effort; encourages a self-compassion, self-kindness mindset while exercising

- Embrace individual differences, encourage unity, and respect of self and others

- Focus on individual goals of improved health is encouraging - diverts attention away from a singular ideal and opens up the possibility of multiple ideals, individual ideals, individual differences

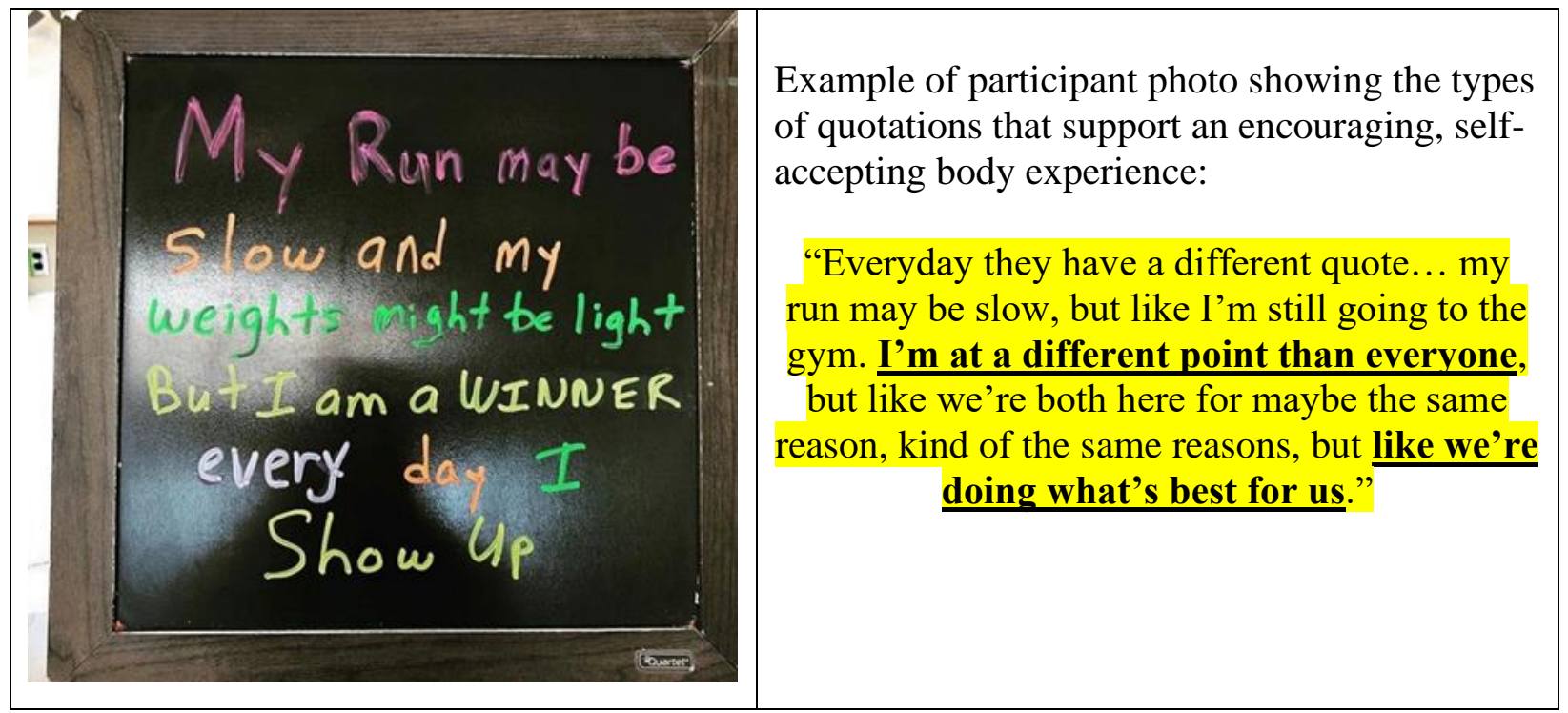

Navigating Their Place

Easing into the Fitness Center Environment:

- Relied on a companion, or ally, to help her gain entry into the fitness center because of fear and intimidation

- Started with cardio early on - described cardio machines as safe, familiar, contained, controlled

- Independently exercising in the fitness center was a threat to her competence - lack of experience and knowledge created a fear of judgement

Creating New Spaces:

- There continues to be a sense of discomfort in the gym as a whole, but comfort is found is smaller, private sub-spaces. This is determined by the people around her and the types of machines she has access to.

- Crowds/crowded fitness center determines her exercise behaviors. Comfort is found where she perceives fewer opportunities for judgement exist.

Relationship with Her Body:

Body Conflict: prescribes to aesthetic ideals but values health and wellness. Body conflict present in several ways: 
- Conflicted (confident but never satisfied)

- Would change to be thinner

- Ambivalence is decreased in fitness center when she finds comfortable space (congruence between self and sub-space she creates)

- Because body conflict is prevalent, seeking comfort in the fitness center is critical for being comfortable in her body

\section{Reflections on the PhotoVoice Experience}

Engaging in the research project itself provided an opportunity to build awareness of the fitness center environment in several ways:

1. Fitness centers CAN provide a setting that offers companionship for women rather than competition and comparison, but many don't

2. Awareness that the fitness center environment influences how we define ourselves and our bodies

\section{Case Summary: P7 (Mya)}

Background and Demographics

Age: 19

Type of fitness center: Health and Wellness/PA for All

The Setting Through Their Eyes

The Fitness Culture:

- Dominated on social media to define fit as "barbie body"

- People that don't fit the thin ideal do not label themselves as "fit" regardless of how dedicated and committed to exercise they may be

- Promotes over conformity - "killing myself at the gym,"and "dying at the gym"

- People that fit the ideal are using positive language "great workout"

- The ideal that exists for women:

- Socially inappropriate for women to be seen red faced and sweating

○ Women must look put-together at all times - if not, go somewhere no one can see you

Choosing THIS Current Fitness Center:

- Valued options including "health care," rather than just fitness services

- Believed there were trusted experts that could guide her health

- Group exercise room

- Upon signup, did not believe there were "meatheads" - later observed this here

Culture of Current Fitness Center:

- "Meat heads" in weight room, overweight people on cardio machines - perpetuating the fitness stereotypes 
- Rooms are separate and have dichotomous cultures

- Main weight area - mirrors everywhere are "the opportunity to [be] judge[d]"

- Messaging in weight room consistent with assumption that this is a man's space

- Lack of health messaging for women

The Fitness Experience

Seeking Comfortable Subspaces:

- Intentionally exercises primarily in empty exercise room

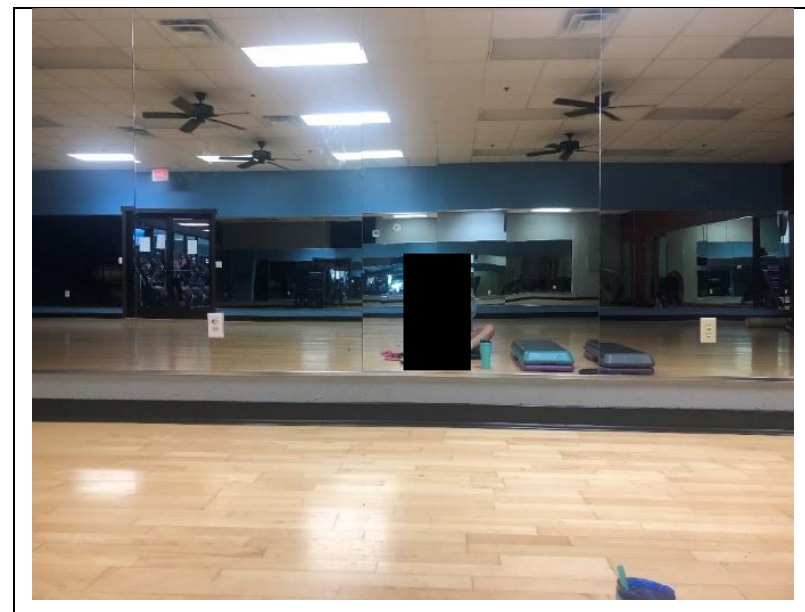

Example of if you don't look like that when you work out, you kind of feel like you got to do it on your own in your own little room.

"You might be doing an exercise that might not be the cutest one, you know glutes, like hip thrusts and stuff are actually - they're pretty awkward to do in front of people".

"When my face gets really red and I'm like dead-sweating, no one can see and so, it's - I just feel like $100 \%$ in my zone and focused".

Forcing women to workout in uncomfortable ways:

- Placement of machines objectifies a woman's body (e.g., lying hamstring machine)

- Treadmills placed so that backside is primary view to main walkway through the gym

- Placement of scale in open area makes her feel "exposed" to the public and modify exercise behavior to exercises perceived to help women lose weight

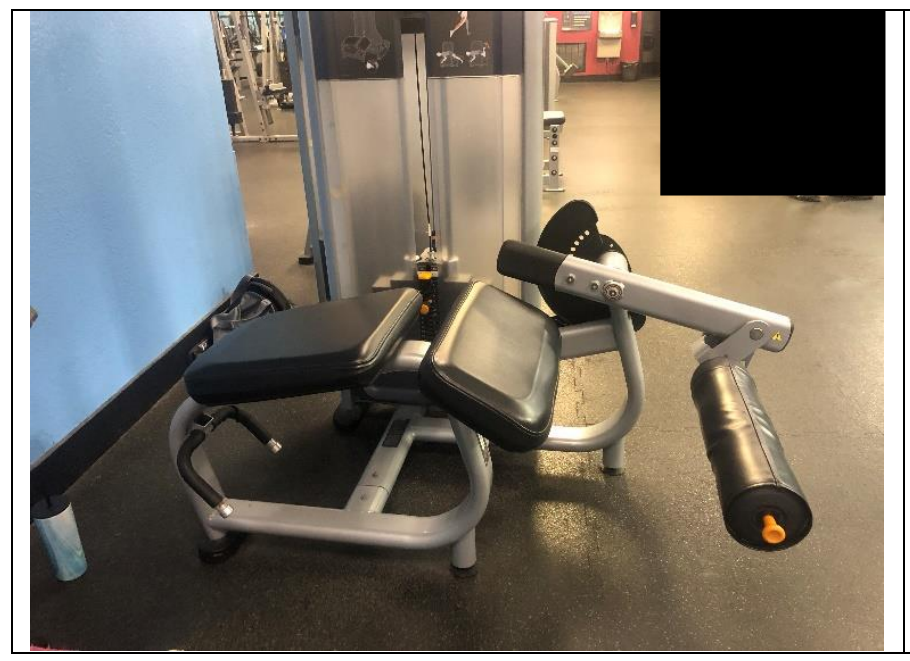

"That is as soon as you walk into the weight-lifting room, it's the first thing.

This actually makes me feel body negative because my butt's in the air for everybody to see, number one."

"I usually try and go pretty quick when I'm on this machine."

"Like I mean at least, face it the other way." 


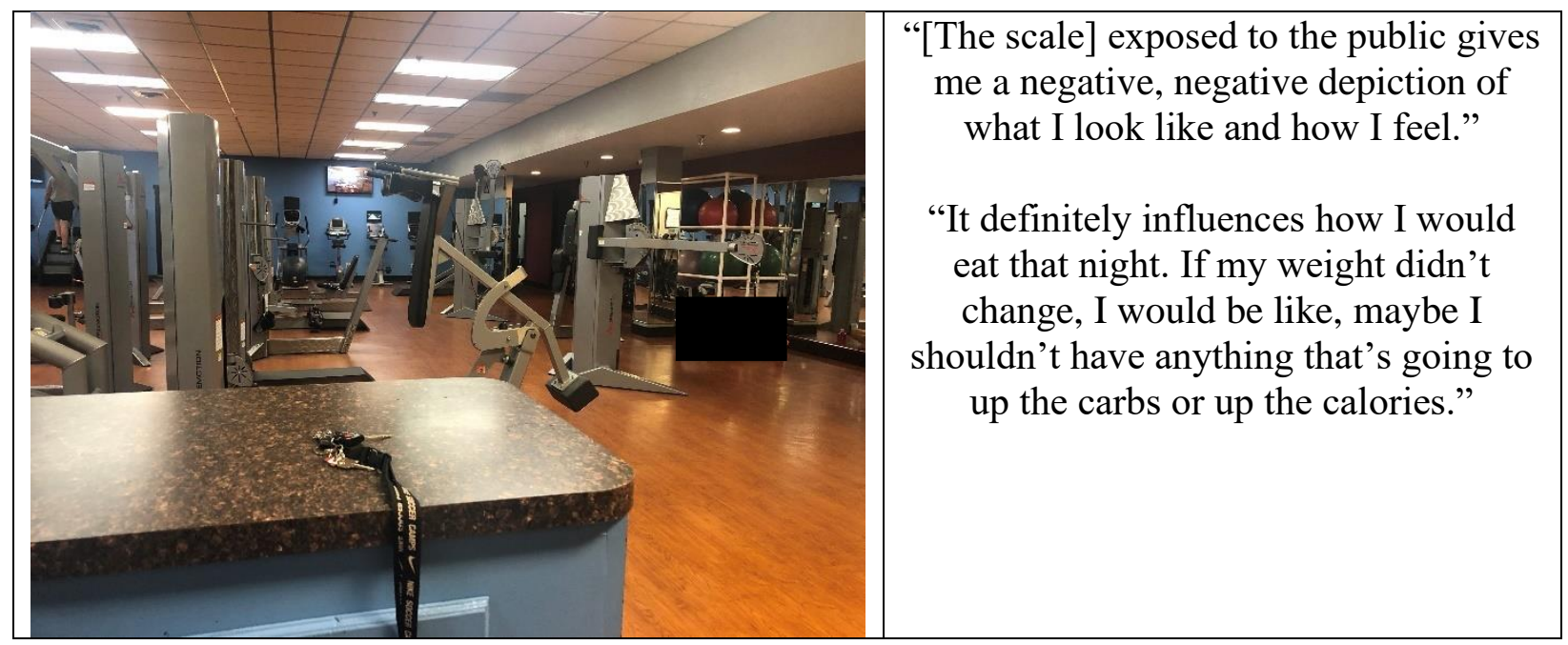

Gender Stereotypes about Strength:

- Male-dominated weight area in room communicating gender norms creates discomfort and self-conscious about her strength

- Not increasing weight on upper body lifts = not strong, not capable, self-conscious.

- Therefore, avoids being seen struggling to lift - threat to attempts to be a strong woman

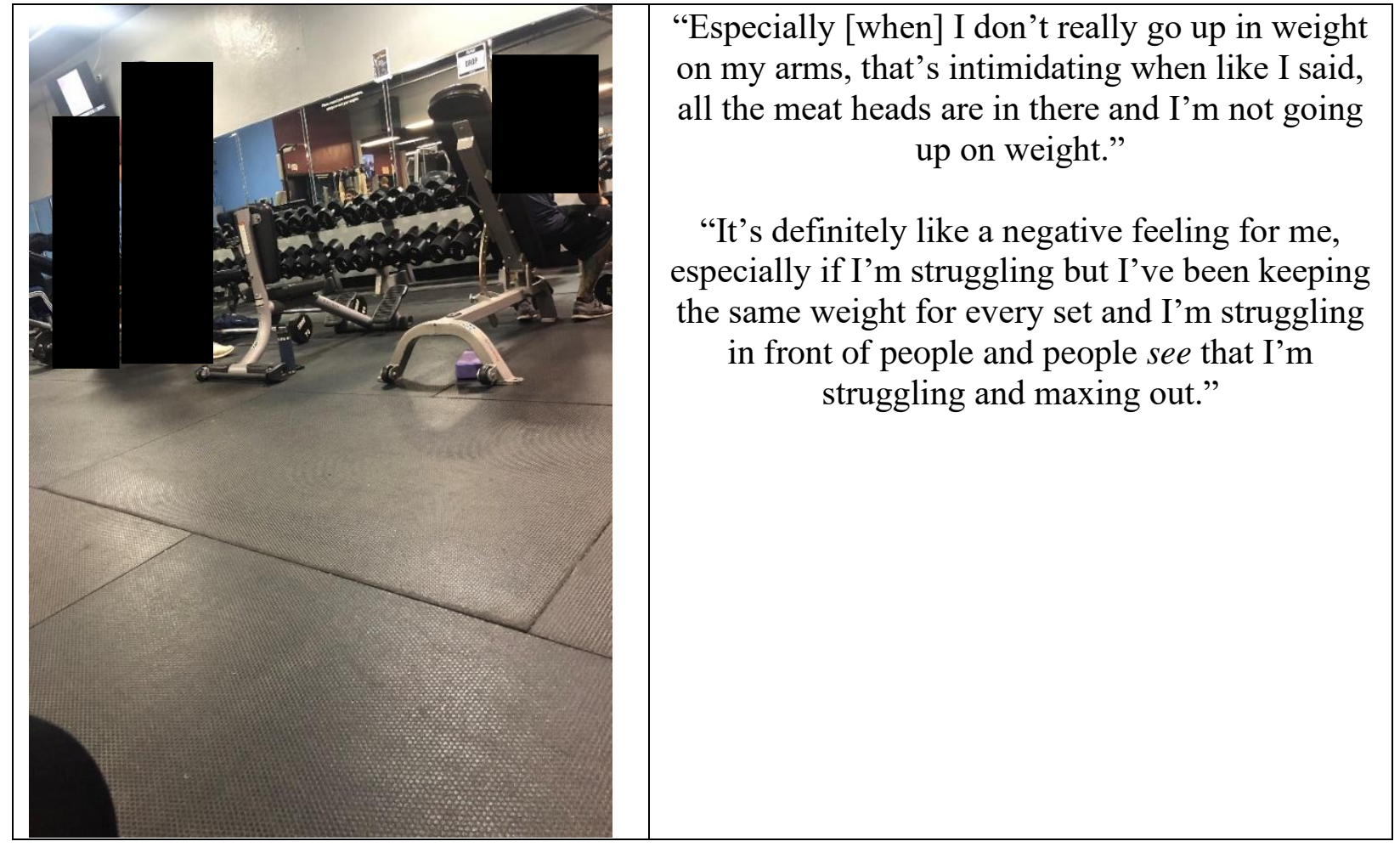




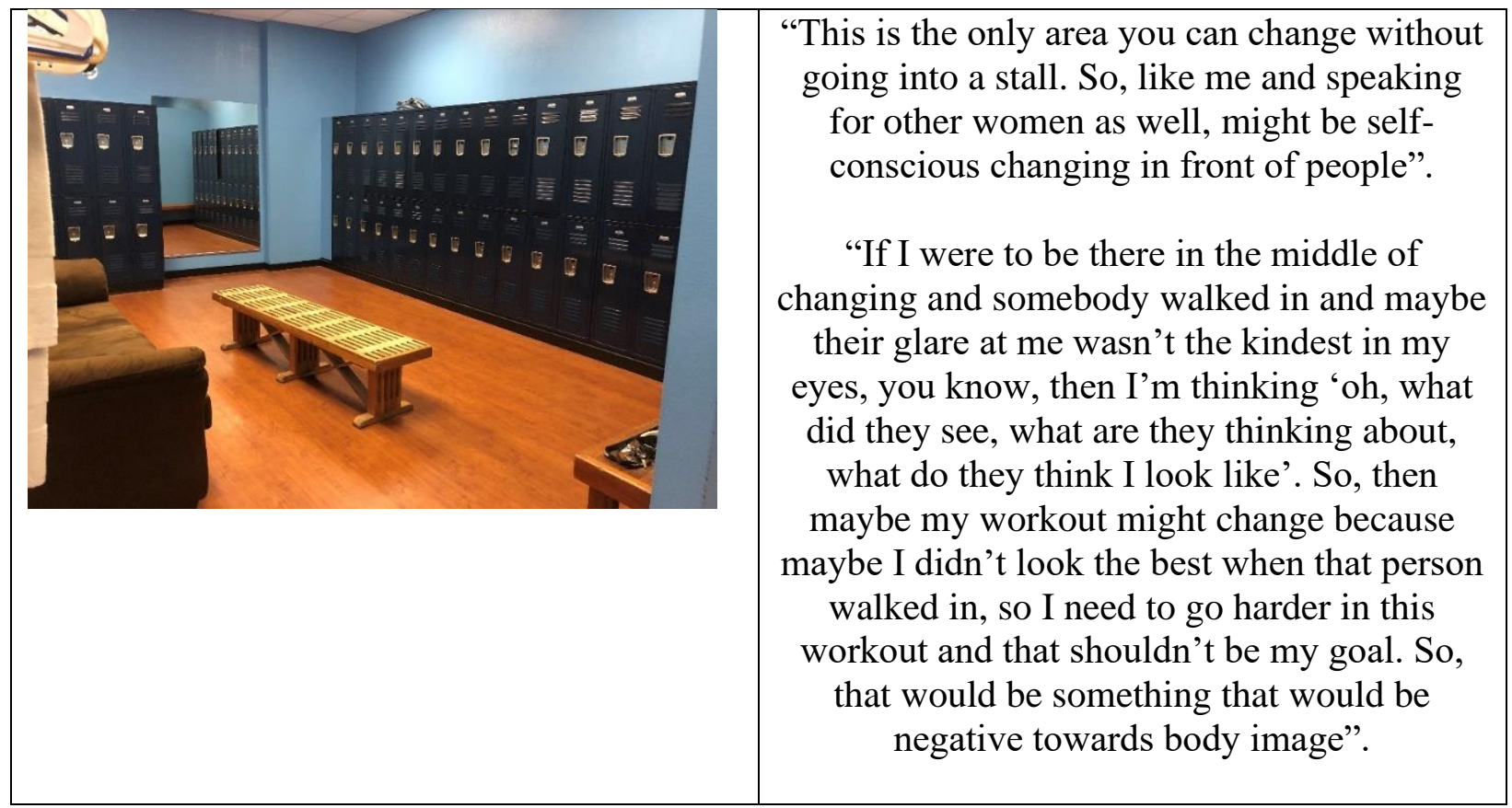

Navigating Their Place

Body Conflict

- Depersonalizes her body and 'segments her body' meaning that her workouts are designed around spot reduction, or training singular body parts to achieve a certain look

- Confident when she is alone and sees definition in her muscles $\rightarrow$ self-conscious when gym is full of "huge guys" and feels watched

Pursuing the Ideal

- Prescribes to overtraining mentality - even when "dead" still "needs to go" to the gym considering a pre-workout supplement for energy

- Struggle between listening to her body for health but desire to pursue unrealistic aesthetic ideals

- Restricts calories when not exercising to avoid weight gain despite hunger

- She is "starving" in the pursuit of aesthetic goals

- Isolates herself during workouts because private space minimizes the potential for judgement from others - especially when doing an exercise perceived as "not cute"

- Negative conclusions about number on the scale and self, motivate a restriction in calories, "shoulds" and guilt

- Cardio/restrictive diet cycle- ignoring her body needs

- Working very hard to change her anatomy to fit the established idea - dimples on hips are genetic

Modifying Exercise Behaviors

- Weightlifting primarily, body-building style (workouts are dictated by how she wants her body to look) 
- Dictated by crowds/volume of people (private/secluded room free of others eliminates the process of worrying about what others are doing, thinking, seeing)

- Seeks relief by spaces devoid of objectification, need to self-present, dissonance

- Fitness center doesn't encourage confidence for a woman's body - asks “where can I flex?" strip away objectification, self-presentation, societal ideals, negative messaging, judging others, and only THEN can a woman flex and be confident.

\section{Reflections of the PhotoVoice Experience}

Engaging in the research project itself provided an opportunity to build awareness of the fitness center environment in several ways:

- Noticed how built environment influences behaviors, thoughts, and body experience

- Increased self-consciousness during the project. Identified more negative than positive factors related to body experience

- Initially difficult to conceptualize the gym as impacting her body experience

\section{Case Summary: P8 (Eve)}

Background and Demographics

Age: 24

Type of fitness center: Employee Wellness facility

The Setting Through Their Eyes

The Fitness Culture: heavily saturated by appearance focused, and apparel driven body ideals. Permeates into fitness center environments, perpetuating unhealthy social comparison and selfcriticism of body. The fitness culture dictates how women perceive fitness and practice fitness.

The body ideal: Women are expected to be put together in most areas of life and we have to do that in the gym while we sweat and workout as well?

- "We all want to lose like fat and stay toned" though.... Societies message supersedes individual goals

- Messages about the thin/fit ideal area distractions from being proud in our body

Current Fitness Center:

- Company fitness center

- Has murals of people doing different activities outside of a fitness context

- Murals depict "healthy" average individuals

The Fitness Experience

Initial experiences with fitness:

- Relied mostly on cardio because of comfort with running

- Tried weights intermittently 
THIS gym:

- Strong, courageous, happy in her skin when exercising

- Safe, comfortable, accountable in her skin at THIS gym

- Safe and comfortable in small space with like-minded patrons

Fitness is a Tool:

- Images throughout the facility of activities other than exercise communicates that exercising here is meant to support capacity to do other functional things in life

Photography example of
life activities:
encouraging images that showcase
"I work out this way so I can
participate in other activities."
"Again, reminding like - being
more aware of the murals that are -
are on the walls - recognizing that
okay I'm building strength for
other activities too."

My Workout, My Choice:

- Private space with options of equipment is empowering and comfortable - free to express self through exercises of choice

- Options provide opportunity to express how women feel in their skin (or want to feel) through exercise

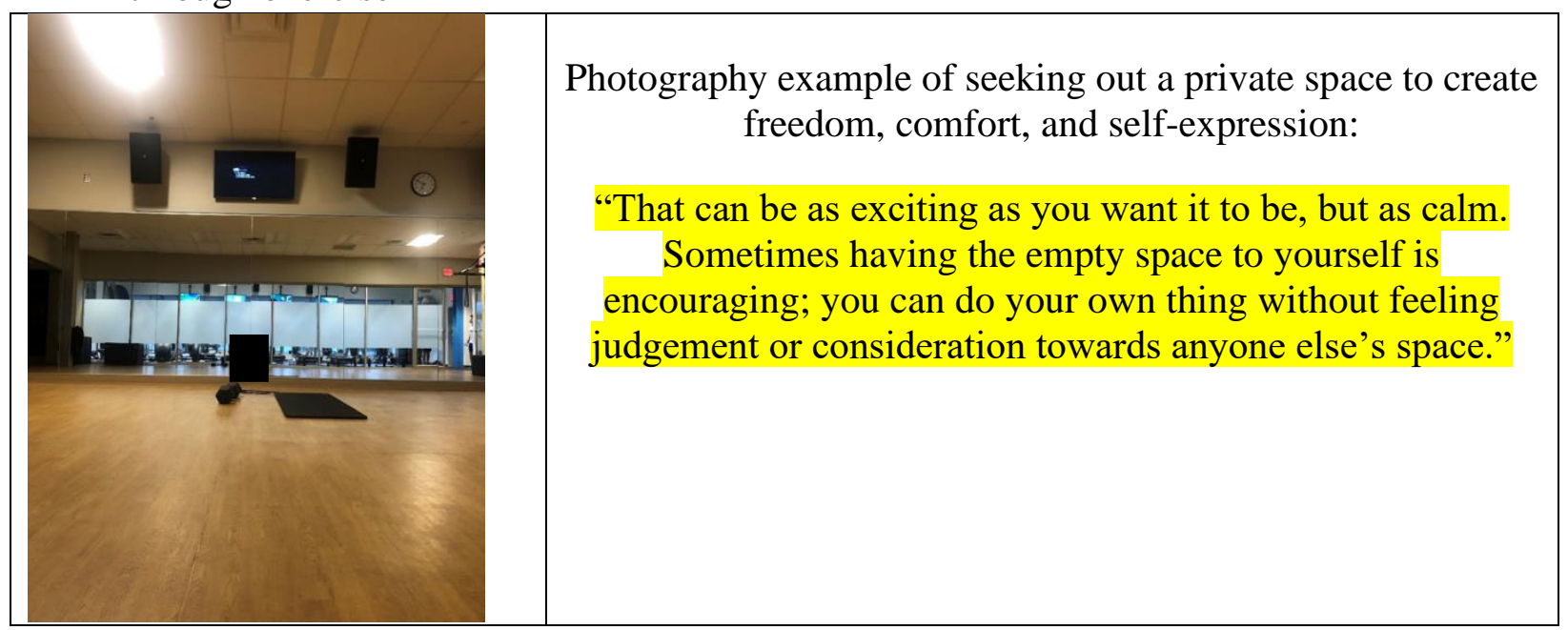

Gender Stereotypes: stereotype that women do not possess upper body strength is represented by certain machines in the main gym area, such as the bench press. Self-consciousness experience when assuming that she is being perceived as weak. 


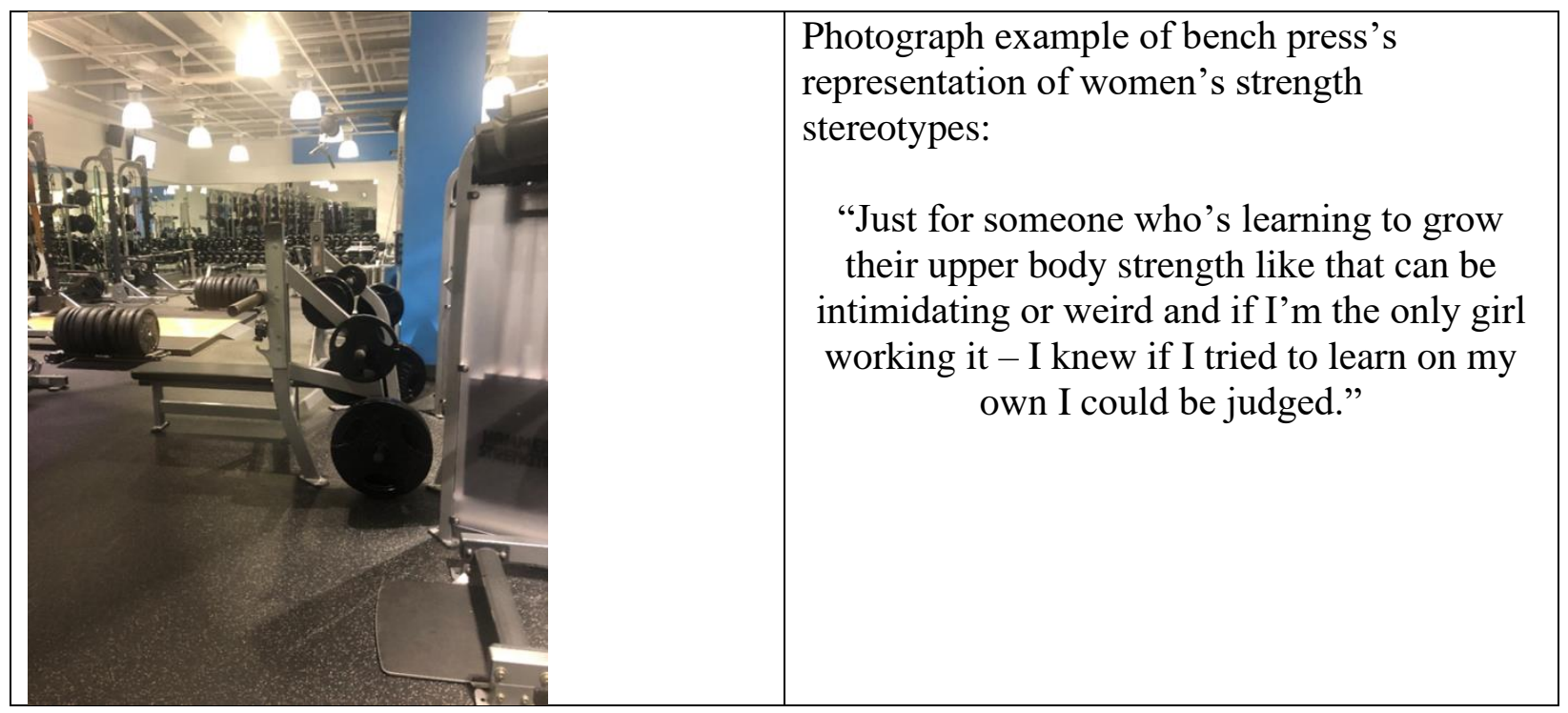

Navigating Their Place

Cautious to Enter Fitness Center: assumption that others are judging young women who are beginners with fitness, this fear of being judged when trying to learn new exercises on one's own is discouraging.

Seeking Privacy:

Fear of judgement can lead to a desire to avoid the more difficult/complex exercises because it might "expose weakness" or seek out a private space which minimizes judgement, comparison, self-presentation. Creating her own space allows her to connect more with her authentic exercise identity.

Relationship with body:

- Proud but struggles to be because of what the media projects

- Past partner commenting on body was unacceptable

- Respects what her body is capable of doing

Reflections of the PhotoVoice Experience

Engaging in the research project itself provided an opportunity to build awareness of the fitness center environment in several ways:

- Realization that the gym influences how we view our bodies and how we exercise in response to those thoughts

- A built environment has power over how we feel in our skin (it's an enmeshed experience), which ultimately influences our behavior towards our body

- When exercise becomes a part of identity - environment has less power over experience and being uncomfortable

- An environment meant to inspire self-improvement, strength, power, health should be the last place a woman feels the need to hide 
Recommendations:

- Women need reminders that we are building strength for life's activities

- Provide a group context - discomfort in weight room when alone can be shifted to an encouraging experience when provided with peers

\section{Case Summary: P9 (Zoe)}

Background and Demographics

Age: 18

Type of fitness center: Appearance-focused, Body Sculpting

The Setting Through Their Eyes

The Fitness Culture:

- Clouded perception of what it means to be fit - appearance is primary definer

- "Quick fix" culture drives people to only want to appear fit rather than be open to learning the craft

- Women prescribe to fitness model ideal (wear the clothes even if not attending gym frequently)

- All women exercise to fix some part of them that society tells them to

- All women have internalized appearance focused exercise motives to some degree

- Easy to identify a fit ideal: can identify specific fit-ideal representing strong, powerful, muscular physique

- Trends change quickly and yet have strong influence on our behavior

- Fear of being "found out" - little room to make mistakes - preserve self-presentation

- HOWEVER, there is a subculture where strength is accepted for women and this teaches there are several ways to be strong

Physical Space of Current Fitness Center:

- Wide range of equipment, functional area, studio for group classes, weight area

- Independent weight lifting room - different culture than main area - no mirrors, autonomy over music, different equipment, scaled equipment

- Design of layout prompts patrons to choose cardio or weights, not both, segregated as traditional fitness center

- Gendered equipment:

○ Underrepresentation of equipment to support females (E.g., 45-lb barbells vs 35lb barbells)

- Not equal representation communicates message of unequal opportunity

- Communicates male preference and predominance

- Group exercise rooms created for women, weight room created for men

The Fitness Experience

"You've already turned away like 90 percent of women" 
Creating equal opportunity - weightlifting room is dominated by women - create a supportive space, attracts women to weights

"I don't know why they won't just buy women's
bars. So, we'll like all show up like early to
make sure we get a women's bar."
"It kind of pushed the idea that it's a men club.
Like, this is a place for like guys to come and lift
and like get swole and, you know, a girl at the
rig like sticks out like a sore thumb."
"The first class that I came to [in the weight
lifting room], I was like, oh, this is going to be
all like super jacked guys and it was like 90
percent women and like two guys. And so, it's
just interesting because like, in environments
where they literally make it easy for girls to
come, like girls show up."
"You're going to run into like making people
feel, just like turning people away, where it's
already like a men's club. So, you've already
turned away like 90 percent of women, and then
you're also turning away, you know, a
population that, you know, is attempting to work
out and needs to."

\section{Navigating Their Place}

Relationship with Her Body:

Embraces discomfort with her body, growth mindset, and orients towards setting goals and achieving mastery in her weightlifting. Experiences body conflict consisting of frustration with nutritional barriers and inability to fuel her body correctly for functional improvements she aspires to achieve.

Seeking out Comfort:

- Discomfort in main fitness area motivates a desire to seek out comfortable subspace. Her current fitness center has a specific weightlifting room where she is comfortable and connected in her own skin

- In this subspace, making mistakes is accepted and therefore does not fear failure or threat to competence

Avoidance: certain exercises are avoided out of fear of confirming stereotypes about women and strength - specifically pull-up machines. 
Reflections of the PhotoVoice Experience

Engaging in the research project itself provided an opportunity to build awareness of the fitness center environment in several ways:

- Therapeutic to take photos of internal experience

- Name it to tame it - factors lose power when identified

- Traditional gyms don't say much about women at all (specifically new exercisers)

- Designed to put women elsewhere

- History of fitness centers - created for men by men, women were an after thought

- Traditional fitness center creates dichotomy - zone out or hyperaware - not being present

\section{Case Summary: P10 (Ann)}

Background and Demographics

Age: 20

Type of fitness center: "Functional Fitness"

\section{The Setting Through Their Eyes}

The broader fitness culture: 'no room for young women who don't look like, or prescribe to, the ideal'

- Discussed the general culture in terms of how it affects others, but does not personally feel the effects of negative appearance-focused fitness culture

- Current fitness culture can be intimidating because it's uncomfortable to ask for guidance and admit that you are not knowledgeable about something as mainstream as exercise

- Perception that fitness clothing communicates opposite message than intended - you first must look good before wearing clothes to workout

- Intimidating environment initially - if one can be brave, it can become encouraging and fun

The Physical Space of Current Fitness Center:

- Small space, designed like conventional fitness center

- Natural light

- Mirrors all over the gym (treadmills away from mirrors to eliminate self-criticism)

- Posters with conduct rules

- Lack of visual portrayals of the thin/fitness ideal

The Fitness Experience

Cautious to Enter the Fitness Center Environment

- Intimidating experience to start exercising in a fitness center, particularly when entering the setting on her own

- Became less intimidating with an ally 
"Get's my Creative Juices Flowing"

- Small gym, with limited equipment creates a challenge to find new ways to get an effective workout

- Empowering experience to be creative, develop personal workouts, and freedom of choice over how and why we exercise

More than a Number:

Scale in the fitness center is a neutral experience for her because she is informed about how weight can fluctuate for many reasons out of one's control. Has developed a health relationship because she has been educated to disprove societal messages about women and weight.

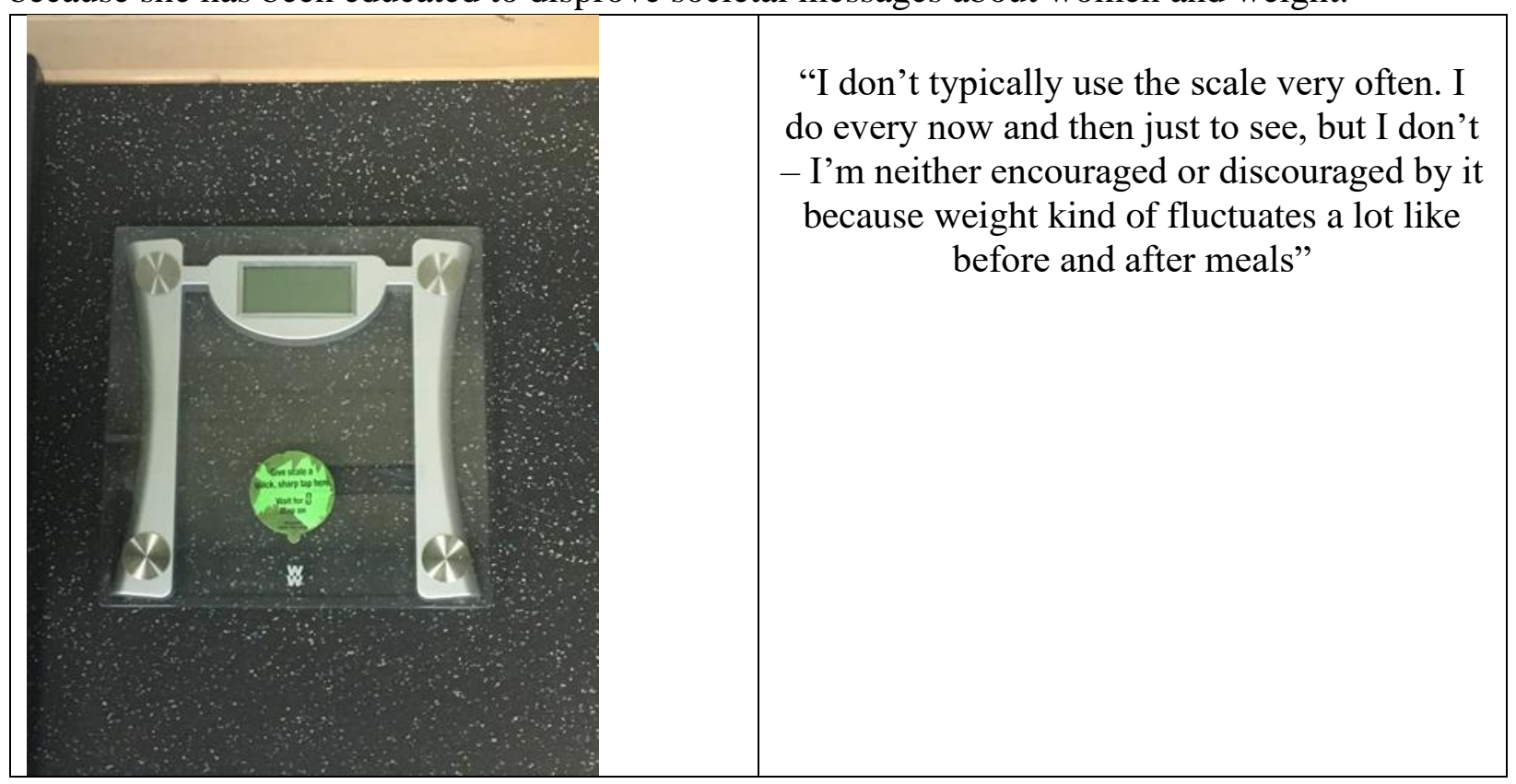

Navigating Their Place

Relationship with her Body: "I love it, it's the only one I have, I want to take care of it"

- Does not prescribe to societal message that women should be put together at all times feels prettiest when sweaty

Self-Concept: "Healthy people aren't miserable with decisions they make"

- Healthy because she balances nutrition, exercise, and recovery

\section{Reflections of the PhotoVoice Experience}

Engaging in the research project itself provided an opportunity to build awareness of the fitness center environment by:

- Increased empathy for others who may have differing (more negative) experiences and how she might contribute to a more positive environment for others

- Gratitude for her healthy mindset and approach to exercise 


\section{Case Summary: P11 (Kay)}

\section{Background and Demographics}

Age: 24

Type of fitness center: Employee Wellness Facility

\section{The Setting Through Their Eyes}

Describing the Fitness Culture:

- Immediately thinks of social media fitness models. Message to look one way, and quick fix tips to get there - unrealistic ideal with unrealistic behaviors

- The fit ideal - would "love" to look this way despite awareness of unrealistic nature of the expectations; forced to live in a state of constant dissonance

Prior fitness Centers: separation of areas with weights in designated "male" zone

Physical Space of Current Fitness Center: describes a variety of equipment in group exercise space (preferred spot), weightlifting platforms and weights in one area, cardio in a separate area. Visuals around the fitness center depict people engaged in physical activity OUTSIDE of fitness center - life activities that are active, as well as banner at the entrance that communicates health and wellness objectives. Fitness center offers community-engagement challenges (attempts to foster social support and relatedness).

Culture of Current Fitness Center: diverges from a traditional fitness center (would not have attracted her if not for her place of employment because it favors wellness/health initiatives like a senior's home).

\section{The Fitness Experience}

\section{Barrier to Entry:}

- Uncomfortable inserting self into weight area - sense of being in the way - lack of belonging

- Around the lifting platforms: being seen as a woman fitting in to a man's world holds a certain status!!! Desires to enter for that status, but doesn't feel a sense of belonging

- Women in a man's world - regardless of competence, she feels in the way in the squat racks -should leave and go use more female-appropriate machines (E.g., dumbbells) 


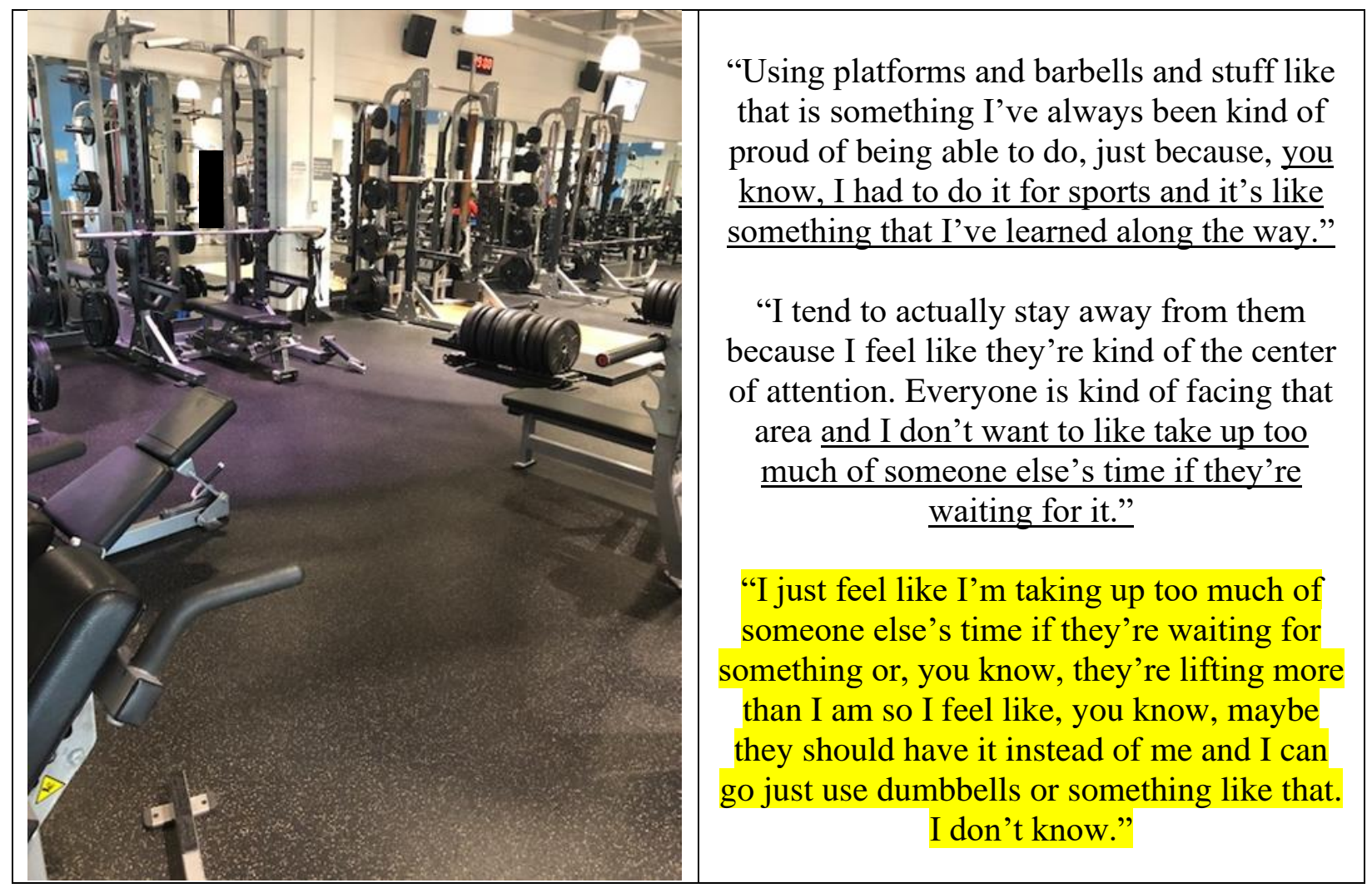

Women and Pull-ups: "Qualifies you as a fit person"

Gendered exercises such as pull-ups represent gender norms about physical strength. Afraid to be seen "failing" to do a pull-up (reinforcing gender norms). The assisted pull-up machine is a salient negative body experience because of the conclusions she makes about her body as a result. 


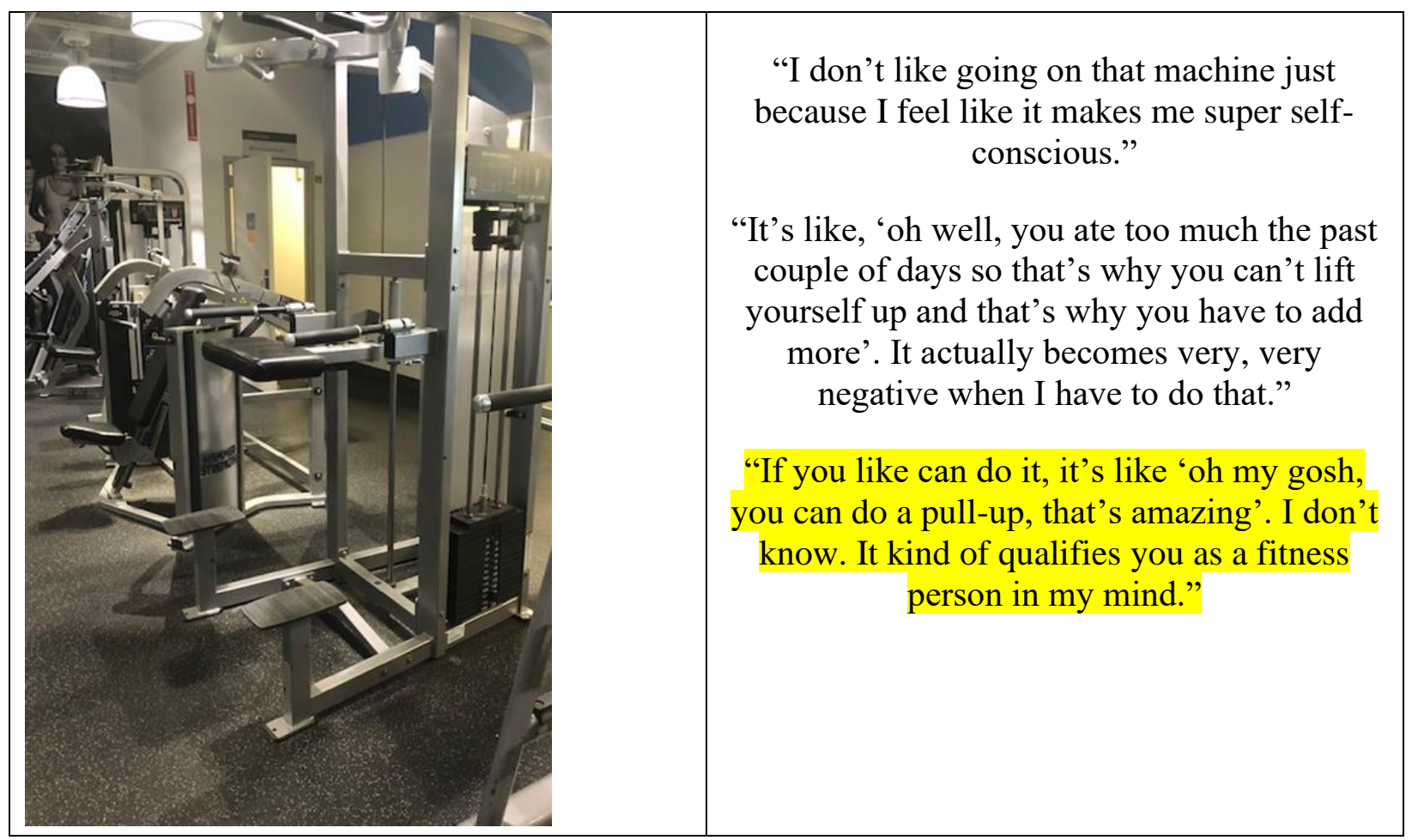

Navigating Their Place

Preferred subspace to exercise is in the group context, with group exercise classes, which creates community, belonging, and challenge. Majority of group context is female, uncomfortable in gendered exercises in gendered spaces - "a woman in a man's world."

Relationship with Her Body: contingent upon doing all the behaviors deemed healthy by society - nutrition, weight on scale going down, how she compares to the ideal. Perceives positive body image on days when she has successfully completed healthy behaviors, and negative body image when feeling weak, ate too much food, weight on scale increases, lack of discipline.

\section{Reflections of the PhotoVoice Experience}

Engaging in the research project itself provided an opportunity to build awareness of the fitness center environment in several ways:

- Fitness center is male-dominated environment - there is a sense of pride if you are a woman, can walk into a male-dominated environment and hold your own, in strength, knowledge, or courage

- Fitness centers are a tool to use to work towards better physical and mental life

- Traditional gyms promote appearance focused motives to exercise to young adult women 


\section{Appendix D: Extended Literature Review}

In the last decade, sociocultural and environmental factors that contribute to negative body image have been increasingly explored across domains including clinical populations of girls and women with eating disorders (e.g., Miyake et al., 2010), among overweight and obese populations, (e.g., Brewis, Sreetharan, \& Wutch, 2018), and, most prolifically, within sport contexts for female athletes (e.g., Reel, Petrie, SooHoo, \& Anderson, 2013). The current literature review will lay the foundation to justify the need to apply this knowledge to the context-specific experiences of young adult female exercisers within fitness center environments. The literature review will begin by introducing body image and the significant influence of sociocultural factors. This will be followed by an exploration of seminal work on weight pressures - referred to as body pressures in this document - in sport contexts to serve as a foundation by which to understand how pressures may similarly be studied in exercise settings. Next, the focus will shift to the exercise context and what is known about weight stigma, a specific body pressure experience by overweight and obese populations. Together, the abundance of literature in body image, weight pressures in sport, and weight stigma in obesity will serve as a template by which to examine these constructs within young adult women in fitness center environments.

\section{Body Image}

Body image is a multidimensional construct of embodiment that includes an individual's perceptions, cognitions, affect, and behaviors towards their body (Cash, 2004). Cash's early work in the phenomenon of body image expressed that the psychosocial experience of body image is often more powerful than the 'reality' of one's physical appearance (Cash, 2004, p. 1). Body image exists along a continuum from healthy body perceptions (i.e., mostly positive and 
accurate) to unhealthy body perceptions (i.e., mostly negative and inaccurate; Cash, Phillips, Santos, \& Hraboshy, 2004; Grogan, 2010). The perception component of body image is conceptualized as the accuracy of one's judgement of their size, shape, and weight relative to their actual proportions (Cash, Wood, Phelps, \& Boyd, 1991). The cognitive component has been conceptualized as the thoughts and beliefs one carries towards their shape and appearance, whereas the affective component includes one's feelings towards their bodily appearance (Cash \& Green, 1986). Finally, the behavioral component is related to presentation habits and grooming behaviors used to maintain one's appearance is response to the cognitions and emotions (Cash, 1994).

In the United States, between 69-84\% of women (Neighbors \& Sobal, 2007; Pruis \& Janowsky, 2010) are dissatisfied with their body. Typically, women report a desire for a smaller size and shape than their current body and resort to dieting to achieve an ideal body shape (Johnson, Edwards, \& Gidycz, 2015). Among adolescent girls and college-aged women, adopting 'fad' diets (e.g., Paleo Diet) is common as a means of eliminating certain foods which are perceived to interfere with weight loss efforts (Radtke, Kaklamanou, Scholz, Hornung, \& Armitage, 2014). Body dissatisfaction is well-established as a predictor of disordered eating symptomatology such as bulimic symptoms and dietary restraint (Neumark-Sztainer, Paxton, Hannan, Haines, \& Story, 2006).

Historically, scientific investigation into the associations between body weight and body image has focused on clinical populations (i.e., eating disorder patients and obesity patients). In fact, body image disturbance has been designated as a central diagnostic criterion of Eating Disorders since the third edition of the Diagnostic and Statistical Manual of Mental Disorders (DSM-V, 2013). By definition, negative body image is a state of subjective dissatisfaction with 
one's physical appearance involving dysphoric emotions, such as anxiety and dejection (Cash, 2002). Negative body image has traditionally been conceptualized as a psychological deficiency of the person with researchers searching to identify perceptual and biological correlates of body image disturbances that are associated with eating disorder symptoms (Miyake et al., 2010). Many empirical studies have used fMRI scanning to study the associations between body image constructs and eating disorders, which further suggests that some researchers hypothesize there to be a 'known' perceptual difference between individuals with eating disorders and those that do not (e.g., Miyake et al., 2010). For example, some researchers quote “... [eating disorder] patients have severe disturbances in the perception of body shape and weight” (Miyake et al., 2010, p. 183). Similar, Boghi and colleagues (2011) wrote, "brain alterations are known to be associated with anorexia nervosa and tend to be distributed across brain structures" (p. 154). In the present landscape, there is a divide in the research community between those examining body image disturbances in terms of perceptual and biological correlates of eating disorders and those who examine more cognitive-evaluative constructs and the influence of sociocultural factors (Murnen \& Smolak, 2019). Contemporary body-image research argues that to fully understand, and improve, body image and associated health outcomes, these phenomena must be conceptualized as fluid, sociocultural, and environmentally-influenced experiences (see Cash, 2012).

\section{Body Image and Sociocultural Pressures}

Western culture is preoccupied with the gendered body (i.e., masculinity versus femininity) and has created a society that emphasizes appearance and beauty as a cultural value linked with identity and self-worth (see Bordo, 2004). Since the 1970s, failure to obtain the unrealistic aesthetic body ideals portrayed by Western culture has led to an increased prevalence of body-image disturbance for men and women (Berscheid, Walster \& Bohrnstedt, 1973; Pruis \& 
Janowsky, 2010). Women often attempt to change their body to achieve the feminine standard of thinness and beauty and although most women will never achieve the cultural body ideal, many relentlessly work toward it through unhealthy diet and exercise (Voelker \& Reel, 2015) Researchers have been fascinated by the influence of cultural beauty ideals on women's body image, affect, and grooming behaviors and have extensively investigated how cultural messages of beauty are communicated to women in contemporary culture.

Sociocultural model of eating disorders. The sociocultural theory is the most empirically-supported theory explaining the acquisition, and maintenance, of body image dissatisfaction (see Stice, 1994). Originally conceptualized as a theoretical model linking sociocultural pressures to bulimia nervosa, Stice (1994) presented a model which included sources of pressures as well as mechanisms that linked sociocultural pressures to disordered eating practices. Stice (1994) delineated sociocultural pressures into three general categories: (1) the thin-ideal body for women, (2) the centrality of appearance in the female gender role, and (3) the importance of appearance for societal success. Stice described the "transmitters of sociocultural pressures" (Stice, 1994, p. 645) to include family influences, peer influences, and media influences through which messages about the thin-ideal, the centrality of appearance to female gender, and the importance of appearance for success are communicated. Stice further described that internalization of sociocultural pressures produces body dissatisfaction in some women which encourages compensatory behaviors believed to result in weight-loss. Internalization is described as the degree to which one adopts a sociocultural body ideal as their own (Thomspon, van den Berg, Roehrig, Guarda, \& Heinberg, 2004). Meta-analyses have demonstrated that three sociocultural constructs - awareness, perceived pressures, and internalization - are all associated with body image evaluation (Cafri, Yamamiya, Brannick, \& 
Thompson, 2005). Interestingly, research by Thompson and colleagues (2004) demonstrated that perceived pressures and internalization of pressures shared $50 \%$ of the variance of body image dissatisfaction in a sample of 175 female college students. These results argue that sociocultural body pressures are strong influencers on women's body image perceptions and are communicate through a variety of persuasive sources.

Sources of sociocultural body pressures. The female body, specifically, that is perpetually portrayed through traditional media depicts a thin-ideal (e.g., models, advertisements, actresses) which is often at the extreme low end of body weight for women. The media influence on women's body image is a sociocultural body pressure that has received significant attention in the literature. Pressures to attain specific body weight, shape, size, and appearance expectations are experienced by individuals when they worry about their weight or shape and perceive that others also judge their body to be unacceptable or not enough (Voelker \& Reel, 2015). Media scholars have examined the influence of exposure to media images of the thin-ideal on the body image of women. It is suggested that women engage in upward appearance-comparisons, evaluating their body in comparison to the bodies of women in the media who they perceive to possess superior attributes (Festinger, 1954; Tiggemann \& McGill, 2004). Correlational meta-analyses and experimental studies have demonstrated a positive relationship between media exposure to the thin-ideal and body image concerns (Grabe, Ward, \& Hyde, 2008) and when women were exposed to images of thin-body, average-body, and neutral stimuli, body image concerns were shown to be highest after exposure to media image depicting a thin body-sized woman (Hendricks, Secharan, \& Clayton, 2016).

Of particular relevance to the current study population of young adult women is the influence of social media which possesses numerous unique factors which differentiate it from 
traditional media sources (Fardouly \& Vartanian, 2016). First, social media (e.g., Facebook, Instagram) features the users themselves rather than public figures, models, and celebrities. Second, users typically upload favorable images of themselves, attempting to display the most attractive, idealized-version of themselves. Importantly, social media outlets allow for the augmentation, enhancement, and editing of photographs (using photo editing applications) which allows users to further display idealized, yet unauthentic, profiles. With Facebook specifically, Stronge, Greaves, Milojev, and West-Newman (2015) showed that young adult women who had a Facebook account reported experiencing greater body dissatisfaction than those that did not have an account. Among Instagram users, usage was negatively associated with body satisfaction among college students and those who displayed lower levels of self-esteem were particularly vulnerable (Ahadzadeh, Sharif, \& Ong, 2017). Similarly, Brown and Tiggemann (2016) exposed participants to images on Instagram of attractive celebrities and unknown peers in an experimental study. Their results indicated that participants exposed to ideal images (i.e., thinideal), experienced significantly more body dissatisfaction than those who viewed neutral photographs of landscapes. These results together suggest that exposure to idealized female bodies through media (traditional and social media) represents a significant sociocultural body pressure for women in relation to their body image.

Appearance-based comparisons and their association to body image dissatisfaction may, in part, be related to the degree to which one adopts unrealistic body expectations and body pressures as their own. For example, Hendrickse, Arpan, Clayton, \& Ridgeway (2017) demonstrated that college-aged women who reported engaging in more appearance-related comparisons on Instagram reported a more intense drive towards thinness and greater body dissatisfaction as a result. As research on the sociocultural weight pressures associated with body 
image dissatisfaction and disordered eating behaviors evolved, investigation into the influence of context-specific body pressures developed. Sociocultural and environmental factors that contribute to a negative body image have been increasingly explored within the sport context over the last decade (e.g., Reel, Petrie, SooHoo \& Anderson, 2013), particularly in aesthetic or appearance-focused sports (e.g., Voelker \& Reel, 2015).

\section{Body Pressures in Sport}

A major area of research investigating the sociocultural factors influencing eating disorder symptomatology in sport focuses on athletes' experiences with body pressures, more commonly referenced in this literature as weight pressures. Weight pressure, referred to more broadly as "body pressure" in this document, is defined as real or perceived coercion from significant others, societal messaging, and/or the culture of sport to change or maintain one's body weight, shape, size, or appearance (Reel, SooHoo, Gill, \& Jamieson, 2005). Most body pressure research has focused on collegiate female athletes (e.g., Reel et al., 2013) and those in aesthetic sports (e.g., Voelker \& Reel, 2015). Pressures to meet specific body ideal expectations or physical specifications for one's sport can lead to a variety of unhealthy, compensatory behaviors including dieting behaviors, disordered eating behaviors, and even clinical eating disorders (American Psychological Association, 2013). The prevalence of eating disorder symptomatology within female sport athletes differs based on whether leanness and appearance is emphasized as part of the sport. In previous literature, leanness sports have been categorized as sports that emphasize a lean physique or require a lower weight for aesthetic or weight requirements (Bryne \& McLean, 2002; Kong \& Harris, 2015). Among female athletes in aesthetic or lean-focused sports, the prevalence of eating disorder symptomatology is reported to be $46 \%$ as compared to $20 \%$ for non-lean sports (Thompson \& Sherman, 2011; Torstveit, 
Rosenvinge, \& Sundgot, 2008). This prevalence has shown consistency across levels of competition with a recent study demonstrating that half (49\%) of elite female athletes in aesthetic, or lean, sports $(n=382)$, were at an increased risk of developing clinically significant eating disorder symptomatology (Kong \& Harris, 2015). These findings suggest that female athletes engaged in particular sports may be at an increased vulnerability for eating disorder symptomatology due to the nature and structure of their sport (Sherman \& Thompson, 2009). In sum, research firmly supports that female athletes participating in sports which include a leanness or appearance component to the performance outcome have a higher prevalence of eating disorder symptomatology and clinical eating disorders and, therefore, there are components inherent in the sport environment that increase athletes' vulnerability to engage in unhealthy, dieting behaviors.

Sociocultural model of eating disorders in sport. Literature investigating the association between body pressures and disordered eating among female athletes (e.g., Berry \& Howe, 2000; Byrne \& McLean, 2002; Petrie, Greenleaf, Reel, \& Carter, 2009) inspired the development of a sociocultural model of eating disorders among female athletes (Petrie \& Greenleaf, 2012) adapted for Stice's (1994) original model. The model suggests that unhealthy eating behaviors develop as a consequence of sociocultural pressures about body size, shape, and weight that athletes experience. More specifically, athletes are subjected to potentially conflicting body pressures from the sport context and simultaneously from the broader society. Athletes then internalize these conflicting ideals and expectations. It is suggested that these pressures lead to negative attitudes about their bodies, emotional negativity, and unhealthy eating behaviors. It appears that through internalization, athletes develop higher levels of body dissatisfaction and/or intentions to increase disordered eating attitudes and behaviors. More 
broadly, the model highlights that women reside inside a larger society where body-related and appearance pressures are communicated by important and close others (e.g., family members, friends, and the media) and are mediated through internalization of the communicated body ideals. These internalized ideals then become the primary benchmark by which female athletes compare and evaluate their bodies. The model further suggests that female athletes are subject to pressures in addition to societal body-related ideals within the sport context. This unique environment includes body weight, shape, and size expectations communicated by coaches, teammates, spectators, the media, and judges. As female athletes internalize these sport specific pressures from their environment and evaluate that their current body falls short of the ideal, they may become less satisfied with their own body and engage in compensatory behaviors (e.g., disordered eating behaviors and/or over exercising) in an attempt to pursue the originally communicated body ideal expectations. This model provides a multi-level framework for understanding the complexity of factors involved in the development of eating disorder symptomatology in a sport context, including the social and cultural influencers. The knowledge that the social environment significantly impacts athletes' perceptions of their bodies in sport suggests that there are influencers, or sources, in the environment that communicate weight pressures to female athletes. The preponderance of cross-sectional and preliminary longitudinal evidence suggests that internalizing these sport-related body pressures contributes to negative body image, negative affect, and eating pathology in female athletes (e.g., Krentz \& Warschburger, 2013; Voelker, Gould, \& Reel, 2014).

Sources of body pressures in sport. In addition to physical demands and performance expectations, female athletes in aesthetic sports are subject to evaluations based on the appearance of, and presentation of, their body. With this added pressure to execute difficult 
athletic skills coupled with a judged artistic component, athletes often experience pressures from a variety of sources to diet to maintain a low body weight, size, or shape which may lead to disordered eating behaviors (Reel, Soohoo, Petrie, Greenleaf, Carter, 2010) and other psychological and psychosocial consequences. Reinforcement of the thin ideal for female athletes is often influenced by the social environment. Competitive sport cultures that emphasize the thin ideal generally pressure athletes to conform to established body ideals if they wish to produce a "gold medal" performance (Muscat \& Long, 2008).

Research investigating the social environment as it relates to body ideals and eating disorder symptomatology traditionally draws from Objectification Theory (Fredrickson \& Roberts, 1997; Moradi, 2010; Szymanski, Moffitt, \& Carr, 2011). This theoretical framework "places female bodies in a sociocultural context with the aim of illuminating the lived experiences and mental health risks of girls and women who encounter sexual objectification" (p. 174, Fredrickson \& Roberts, 1997). This theory suggests that the experience of being seen as a body and valued primarily for its consumption and evaluation by others leads to the internalization of sociocultural body ideals (Moradi, Dirks, \& Matteson, 2005). Objectification theory posits that social contexts that accentuate females' awareness of observation, and evaluation, by others (e.g., critical comments by others) in relation to their bodies are predictive of negative emotions and/or disordered eating (Fredrickson \& Roberts, 1997). In the context of sport, researchers suggest the objectification of the female body through the inclusion of appearance-based performance criteria, form-fitting uniforms, and attention focused on judging an athlete's level of leanness negatively affects the body image of athletes (Karr, Davidson, Bryant, Balague, \& Bohnert, 2013). It appears that athletes participating in aesthetic, or lean, sports are not only expected to perform difficult athletic skills, but are also subject to 
sociocultural body ideals which are independent of athletic performance and based solely on appearance and presentation (Petrie \& Greenleaf, 2012). Systematic review on body image research between 1997 and $2012(n=10)$ sought to synthesize findings on beauty ideals, sexual objectification, and body image concerns among female athletes and non-athletes (Varnes et al., 2013). Findings revealed that athletes participating in sports that emphasize aesthetics or leanness (e.g., ballet, cheerleading, gymnastics, figure skating) reported greater body image concerns than endurance or ball-game sports (Varnes et al., 2013). Interestingly, their findings suggest that pressures experienced by female athletes are not only related to performance expectations but extend beyond to sociocultural expectations perpetuated by the media (Varnes et al., 2013). Therefore, it appears that appearance expectations encourage, and reinforce, female athletes to engage in self-objectification which fosters habitual body monitoring, internalization of sociocultural body expectations, associated appearance anxiety, body shame, and the potential development of eating disorder symptomatology (Greenleaf, 2002; Moradi, Dirks, \& Matteson, 2005; Tylka \& Hill, 2010).

Female athletes experience body pressures from a variety of sources within the sport environment which increase their vulnerability to eating disorder symptomatology (e.g., Carrigan, Petrie, \& Anderson, 2015; Cheek, Hill, Carlson, Lock, \& Peebles, 2015; Francisco, Narciso, \& Alarcão, 2013; Voelker, \& Reel, 2015). As research began to investigate the sources of pressures related to body weight, shape, and size for female athletes in aesthetic sports, the need for a psychometrically sound assessment tool to identify sport-specific, weight-related pressures associated with risk of disordered eating was critical. Reel, Soohoo, Petrie, Greenleaf, and Carter (2010) developed the Weight Pressures in Sport for Females (WPS-F). Exploratory factor analysis revealed a 16-item measure with four factors: Weight Pressures from 
Coaches/Team/Sport, Self-consciousness of Weight and Appearance, Importance of Weight and Appearance, and Weight Limit with strong internal consistency (Cronbach's alpha of .90). Prior to the development of this scale, there was no systematic approach for quantifying sport-specific weight pressures for female athletes. With its development, researchers sought to validate and confirm the measure. Reel, Petrie, SooHoo, and Anderson (2013) examined the psychometric qualities of the measure with female collegiate athletes $(N=414)$ in two samples using exploratory $(n=207 ; M=19.27$ years; $S D=1.16)$ and confirmatory $(n=207 ; M=19.19$ years; $S D=1.66)$ analyses respectively. It was revealed that Coach and Sport Pressures about Weight (Factor 1) and Pressures Regarding Appearance and Performance (Factor 2) had emerging reliability and had practical implications for assisting in the identification of weight-related pressures experienced by female athletes in their sport settings.

Some of the most cited sources of weight pressures, as reported by athletes, include: comments from coaches, judges, and teammates, the revealing nature of form fitting uniforms, established body ideals perpetuated by the sport culture, and the belief that a certain weight leads to performance advantages (Anderson, Petrie, \& Neumann, 2012; Greenleaf, 2004; Muscat \& Long, 2008; Reel, 2012; Reel \& Gill, 1998). Many body pressures experienced by athletes originate from, and are reinforced by, significant others in an athlete's life such as parents, coaches, and teammates (Reel, 2012). Findings suggest that coaches that communicate messages focused on appearance and weight are reported by athletes as being significant environmental pressures for female athletes which may be linked to distress and engagement in unhealthy eating behaviors (Muscat \& Long, 2008). Furthermore, many of these pressures are perpetuated by the judging criteria inherent in aesthetic sports (Reel, Soohoo, Gill, \& Jamieson, 2005). For example, Greenleaf (2004) found that 58\% of college synchronized skaters experienced pressures to meet 
body ideals for their sport, while skaters identified the sources of pressures to lose weight to teammates $(57.5 \%)$, coaches $(36.3 \%)$, themselves $(24.2 \%)$, and parents $(12.1 \%)$. Reel and colleagues (2010) surveyed college female athletes $(N=204)$ from three universities across 17 sports representing both leanness and non-lean sports. Within lean- and appearance-focused sports, cheerleaders $(100 \%)$, divers $(100 \%)$, and gymnasts $(100 \%)$ reported receiving weightrelated pressures from teammates.

Coaches were also been cited as a major source of weight-related comments among female athletes. Previous literature indicates that athletes $(75 \%)$ that are told by coaches to lose weight will attempt to do so and most athletes will engage in unhealthy eating practices to accomplish the expected weight loss (Rosen \& Hough, 1988; Stice, 1999). Critical comments from important others are firmly established in the literature as being associated with eating disorder symptomatology (Kerr, Burman, \& Souza, 2006; Lieberman, Gauvin, Bukowski, \& White, 2001; Muscat \& Long, 2008). It appears that comments that consciously, or unconsciously, perpetuate and reinforce current, unhealthy body ideals are highly related to unhealthy eating behaviors (Berry \& Howe, 2000). Furthermore, comments that contain disparaging or teasing content that target an athlete's body weight, shape, or size significantly increase the risk for eating disorder symptomatology (Tiggemann \& Lynch, 2001). Muscat and Long (2008) demonstrated consistent findings when they investigated the associations between the degree of eating disorder symptomatology and level of sport participation, recollection of critical comments made by others, and the intensity of emotions elicited from critical comments directed at female athletes. Participants completed the Eating Disorders Examination questionnaire (EDE-Q), Beck Depression Inventory (BDI-II), and the Social Hassles Questionnaire (SHQ). Consistent with Kerr and colleagues (2006), female athletes who recalled 
receiving critical comments about their body reported greater eating disorder symptomatology, as compared to those that did not recall critical comments. Furthermore, the severity of the critical comments made towards athletes was associated with the level of competition of the athletes and an increase in severity of disordered eating behaviors. In relation to the emotional response to critical comments, female athletes who recalled critical comments made about their body reported more intense negative emotions. Most importantly, the results revealed that the content of the body-related comments including the use of specific terms such as "fat", negatively impacted the athletes. In sum, the above literature highlights that comments that are critical in nature and use specific body-related, negative language result in negative emotional responses and greater eating disorder symptomatology.

Prior research investigating the prevalence rates of disordered eating in athletes has generally sampled from categories of sports (e.g., ball sports, endurance, aesthetic) which has provided limited ability to capture differences on a sport-specific basis. Hausenblas and Carron (2002) suggested that research focus on the study of individual sports with the intent of providing insight into single sport prevalence rates. Among gymnasts, early research suggested that weight control behaviors were predicted by social pressures to be thin (Berry \& Howe, 2000) communicated by coaches, judges, and peers (Sundgot-Borgen, 1994). To further understand gymnasts' experiences, Kerr and colleagues (2006) elicited the perspectives of current gymnasts, retired gymnasts, coaches, parents, and judges to investigate weight management behaviors in the sport of gymnastics. Participants completed surveys uniquely designed for the study which included open-ended, short-answer questions, Likert scales, and checklist items. A significant finding from the study was that disparaging comments made about a gymnast's body had a powerful influence on the gymnast's body image and the engagement in 
unhealthy weight management strategies. Interestingly, it was comments from coaches that athletes were most likely to believe, internalize, and consequently begin to engage in unhealthy weight management behaviors. Furthermore, gymnasts that received disparaging comments from coaches were more likely to report eating disorder symptomatology as compared to gymnasts who did not receive negative comments about their body.

To date, the majority of research examining associations between weight pressures and unhealthy weight management practices in sport contexts has been self-report and cross-sectional in nature. Anderson, Petrie, and Neumann (2011) suggested that longitudinal and experimental research is needed to better understand the temporal relationship between sport-specific body pressures, body dissatisfaction, and engagement in unhealthy eating behaviors. Anderson, Petrie, and Neumann (2012) longitudinally tested, using a cross-lagged model, the pathways between body pressures from the sport environment, satisfaction with their body, and athletes' intention to restrict their eating among NCAA Division I gymnastics, swimmers, and divers $(N=325)$. A 20-item weight pressures scale modified from Reel \& Gill (1998), a 7-item Body Factor subscale from the Body Parts Satisfaction Scale-Revised (BPSS-R; Petrie, Tripp, \& Harvey, 2002), the 9item Dietary Intent Scale (DIS; Stice, 1998), and the 10-item Dutch Retrained Eating Scale (DRES; Van Strien, Frijters, Van Staveren, Defares, \& Deurenburg, 1986) were administered at two times points: (1) at the beginning of the fall season, and (2) at the end of season within two weeks prior to the team's conference championships. Over the 5-month study period, the results indicated stability of athletes' experiences of body pressures, body dissatisfaction, and restrictive eating behaviors. More specifically, the authors concluded that initially reported body pressures and dietary eating attitudes are the best predictors of what is experienced in the future. Further, the authors suggest that the stability of the athletes' experiences of body pressures may be related 
to the environment in that they are constantly training and competing in the context where messages about their body and weight are stable, resulting in a consistent focus on their bodies, weight, and their eating behaviors. These results confirmed that by being continuously exposed to body pressures, disparagement, and scrutiny from individuals in the sport environment (i.e., coaches and teammates), there is an increase in body dissatisfaction over the duration of a competitive season for female athletes.

In conclusion, there is consensus within the literature that female athletes participating at high levels of competition, particularly in aesthetic sports, or sports that emphasize appearance, are susceptible to an increased risk of eating disorder symptomatology. In exploration of the underlying mechanisms, research reveals that female athletes are subjected to social and cultural expectations that objectify the female body. Many of these social expectations are communicated to female athletes through body pressures such critical comments or messages about body weight, shape, or size from significant others such as coaches, teammates, and judges. These comments are internalized by athletes, fostering body dissatisfaction, and encourage the desire to engage in unhealthy weight management practices to pursue a socially constructed body ideal. As research continues to support these dynamics, there may be potential to expand these concepts into other contexts in which women experience socially-initiated body pressures and the psychological and physical impacts of those experiences.

\section{Body Pressures in Exercise Contexts}

Exercise has the potential to be an empowering means by which women can challenge themselves, gain a sense of strength in identity, and learn the breadth of their physical capabilities. However, exercise also has the potential to be coercive and restricting if women engage in exercise as a means of pursuing an aesthetic body ideal (Krane, Waldron, Michalenok, 
\& Stiles-Shipley, 2001). Cash, Santos, and Williams (2005) identified two coping response styles that individuals may use to manage body pressures and ideals. Avoidant (attempting to avert or escape body image-related threats) and appearance fixing (engaging in efforts to alter appearance by covering, camouflaging, or correcting the perceived flaw) were defined as less adaptive coping styles. A more adaptive coping response identified by the authors was positive rational acceptance (accepting the distressing event and engaging in self-care and rational self-talk). As previous research has determined, the cognitive appraisals that individuals make about perceived body pressures present in the environment and the internalization of body expectations typically inspires one of the above three coping responses. Current trends in female body ideal media, including women's magazines and social media, highlight “fitspiration” which brings women's focus to 'problem' areas or 'trouble spots' which can be 'fixed' with easy-to-do exercises that are promised to reshape the body for a more attractive physique (Prichard, McLachlan, Lavis, \& Tiggemann, 2018). Unfortunately, these messages have a history of communicating contradictions such as "firm but shapely, fit by sexy, strong but thin" (Markula, 1995, p. 424) which, for some women, induces negative coping responses. Further compounding the effects of contradictory body pressures in exercise contexts is the dissemination of popular "fitspiration content”. Fitspiration is defined as content promoting fit/healthy lifestyles (Hendrickse et al., 2017). However, much of the fitspiration content includes objectifying images of women's bodies accompanied by messages encouraging dieting, normalizing compulsive exercise, and guilt-inducing messages (Boepple \& Thomspon, 2016). Hendrickse and colleagues (2017) assessed that current fitspiration websites share many commonalities to traditional "thinspiration" websites that depict the traditional thin-ideal. It was further described that both fitspiration and thinspiration websites include fat/weight stigmatization content, messages 
objectifying the female body (e.g., an image of a women in a sports bra with a measuring tape around her waist) and content related to dieting and restraint.

This trend within the exercise industry is unfortunate because there is literature supporting the positive relationship between exercise engagement and reduced body dissatisfaction (e.g., Campbell \& Hausenblas, 2009; Hausenblas, Cook, \& Chittester, 2008), improvements in body image (e.g., Reel et al., 2007) and increases in positive body image (e.g., Hausenblas \& Fallon, 2006; Homan \& Tylka, 2014). However, factors that may prove critical in the exercise-body image relationship are frequency of exercise, age of the exerciser, and reasons for exercise engagement. For example, in young women self-esteem and body satisfaction has been shown to decrease with increases in amounts of exercise (Tiggemann \& Williamson, 2000). Furthermore, BMI has been consistently investigated to have a moderating role (e.g., Barker \& Galambos, 2003), suggesting that the connection between body image concerns and weight management behaviors (e.g., over exercising and restrictive dieting) is complex and does not represent a direct relationship. Evidence seems to point to BMI as a consistent predictor of adolescents' body dissatisfaction (e.g., Barker \& Galambos, 2003; Lawler \& Nixon, 2011; Rosenblum \& Lewis, 1999; Tiggemann, 2005) which introduces questions of how girls and women across BMI classes experience sociocultural body pressures. Overweight and obese women have been a subpopulation of women in which research has focused on the influence of sociocultural weight pressures on physical activity behaviors - namely weight stigma.

Weight stigma. Being an adult with excessive body weight brings significant physical, social, and emotional challenges. One specific area of body-related social constructs in exercise contexts focuses on the social stigma overweight and obese individuals are subjected to and subsequent consequences of these experiences. Overweight and obese individuals are subject to 
negative stereotypes and are often described as lazy, unattractive, and lacking control (Brochu \& Esses, 2011). Weight bias is defined as "negative weight-related attitudes, beliefs, assumptions, and judgments toward individuals who are overweight and obese (and weight stigma) is the social sign that is carried by a person who is a victim of prejudice" (Washington, 2011; p. 1). Unfortunately, weight stigma is pervasive in current culture and yet, it is described as the last “acceptable" form of bias (Puhl \& Brownell, 2001). Weight stigma commonly includes the existence of, and expression of anti-fat attitudes active in social situations which, for those exposed to weight-based discrimination, can have harmful psychological, emotional, and behavioral effects. As obesity continued to escalate as a public health concern, research began to focus on finding consensus among the associated psychosocial factors of weight stigma. In an early comprehensive review of literature, it was revealed that individuals experience unfair treatment in several areas of life such of employment, health care, and education (Puhl \& Brownell, 2001). At that time, the authors noted that much of the published research focused on the objective, behavioral consequences of weight stigma, but few studies investigated the emotional and psychological effects. Several years later, Puhl and Heuer (2009) conducted a similar review of literature in hopes of providing insight into the current knowledge regarding the psychological, emotional, and physical effects of weight stigma. Research conducted between 2000-2008 confirmed much of the previous research findings that suggest weight stigma continues to be of concern in areas of health care, education, and employment from a variety of sociocultural sources.

Sources of weight stigma in obesity. Friedman and colleagues (2005) reported that greater than $75 \%$ of obese individuals seeking weight-loss treatment $(N=93)$ experienced weight-based stigmatization from a variety of sources. Commonly reported sources in which 
obese individuals experienced stigma include negative comments from family $(97.7 \%)$, physical barriers $(96.8 \%)$, inappropriate comments from doctors $(89.1 \%)$, hurtful comments from others (89.1\%), loved ones embarrassed by their size $(86 \%)$, others making negative assumptions (78.3\%), comments from children (76.3\%), being stared at (57\%), and being avoided, excluded, or ignored (55.9\%). Puhl and Heuer's (2009) review found evidence to support the presence of weight stigma within interpersonal relationships. Obese individuals experience stigma in close interpersonal relationships such as from family members, from friends and peers, and in romantic relationships (Puhl \& Heuer, 2009). Puhl and Brownell (2006) examined the most commonly experienced sources of interpersonal weight stigma within a sample of overweight and obese adults $(N=2,671)$. In addition, they explored participants access to coping strategies, general eating behaviors, and psychological functioning. Participants completed self-report surveys which measured frequency of weight stigmatization, depression, self-esteem, binge eating behaviors, attitudes about obesity, coping responses, and most common sources of experienced weight stigma. Results indicated that $72 \%$ of adult women reported family members as the most frequent source of stigma about their weight. Furthermore, when asked about their experiences, women reported that they were the target of weight-focused teasing, judgmental comments, and name calling by parents and siblings. Additional sources of interpersonal weight stigma reported by obese women in the above study were friends (60\% of respondents) and spouses ( $47 \%$ of respondents). These findings were supported by future research indicating that family members are a major source of weight stigma (Puhl, Moss-Racusin, Schwartz, \& Brownell, 2008). These results indicate that a major source of interpersonally-based weight stigma originates within familial, romantic, and friend relationships. Qualitative studies support these findings as well (Rogge, Greenwald \& Golden, 2004). Rogge et al. (2004) conducted 
interviews with 13 obese individuals using an interpretive phenomenological design and revealed that individuals experienced frequent weight-based stigmatization and discrimination.

Furthermore, individuals expressed that interactions with family members, peers, healthcare provides, and strangers were constant reminders that they were deviants from social weight norms. In addition to suggesting that significant others play an integral part in overweight and obese individuals' experiences of weight-related stigma, research suggests that the quality and presence of close relationships is impacted by weight stigma. One study demonstrated mixed findings regarding the relationship between weight stigma from interpersonal sources and selfreported quality of those relationships. Carr and Friedman (2006) surveyed American adults $(N=$ 3,656) and found no differences across BMI for self-reported quality of interpersonal relationships. They did find that obese individuals reported higher strain on close personal relationships and less support from family members compared to normal weight individuals.

Current understandings about the relationship between weight stigma and interpersonal relationships appear to be complex. Research firmly supports the pervasiveness of weight stigma from interpersonal sources showing that obese individuals experience significant stigma and receive weight-related messages primarily from family members and peers which may impart strain on these relationships and reduced support from family members. These findings suggest that overweight and obese individuals experience weight-related messages stigma within a social context. In sum, it appears that weight stigma in the form of critical comments from others originates from the social context in which individuals reside. Furthermore, these messages influence the quality of interpersonal relationships which may lead to the experience of negative psychological and physical experiences. 
The key findings of the Puhl and Heuer's (2009) suggested that individuals subjected to weight stigma are generally left unsupported with little guidance in coping resources. The authors stated that little attention has been paid to assessing the impact of weight stigma on the psychological and physical health consequences for affected individuals up until this point, although a small body of literature had emerged. What research had been done suffered from methodological limitations such as a lack of reliable and valid assessment instruments, a lack of longitudinal studies examining weight stigma over time, and limited assessment of behavioral expressions in response to psychological effects of weight stigma.

Evidence consistently indicates that individuals who have experienced weight bias, weight-based teasing, and weight discrimination experience psychological consequences such as depression, negative self-esteem, and body image dissatisfaction (e.g., Friedman, Reichmann, Costanzo, Zelli, Ashmore, \& Musante, 2005; Jackson, Grilo, \& Masheb, 2000; Annis, Cash, Hrabosky, 2004). Compounded by findings that suggest victims of weight stigma are left unassisted and with poor coping resources, overweight and obese individuals are at an increased risk for the development of psychological concerns. Some research suggests that weight-based teasing may moderate the relationship between obesity and depression in both nonclinical and clinical samples (e.g., Chen et al., 2007). For example, Chen and colleagues (2007) found that among obese patients seeking weight loss surgery $(N=60)$, those that reported weight-based stigma experiences had higher levels of depressive symptoms and mood, even after controlling for gender, age of onset of overweight, and current BMI. Among community-based samples, results have been similar. Annis and colleagues (2004) found that among overweight women $(N$ $=58$ ), those that experienced weight stigma reported depressive symptoms, regardless of what age they recalled experiencing the weight stigma. Broadening the scope of depressive symptoms 
to include other facets of emotional health, Carr, Friedman, and Jaffe (2007) investigated the relationship between obesity and emotional well-being among a sample of American adults $(N=$ 3,353). Interestingly, they found that individuals with a BMI $\geq 40 \mathrm{~kg} / \mathrm{m}^{2}$ reported maltreatment based on their weight and this, in turn, was significantly associated with negative mood. Their results indicated that the experience of chronic obesity was not significantly distressing, but rather the experiences of mistreatment regarding weight lead to impaired mood. Research in this area suggests that experiences of weight stigma or weight-based discrimination is significantly associated with psychological distress, negative affect, and depression.

Several studies have begun to investigate the influence of weight stigma experiences on body image and self-acceptance in overweight and obese populations. For example, research indicates that there are significant, positive associations between experiences of weight stigma and body dissatisfaction among clinical (e.g., Rosenberger, Henderson, \& Grillo, 2006) and nonclinical populations (e.g., Vartanian, \& Shaprow, 2008). Weight stigma may also mediate the relationship between obesity and self-esteem. Research has consistently demonstrated associations between weight stigma experiences and low self-esteem in clinical populations such as among obese individuals in treatment programs (Friedman et al., 2005), and among bariatric surgery patients (Rosenberger et al., 2006). Carr and Friedman (2005) found that among a nationally-representative sample $(N=3000)$ of adults, weight discrimination fully mediated the relationship between obesity and levels of self-acceptance. Therefore, weight stigma experiences are significantly associated with body dissatisfaction, lower self-esteem, and lower levels of selfacceptance.

Research on body image firmly suggests that body ideals are socially-constructed and therefore are embedded in social and cultural definitions of beauty. With this in mind, literature 
has begun to investigate cultural aspects of body image and body dissatisfaction in non-Western cultures with the intent of exploring cultural differences of weight stigma experiences within differing definitions of body ideals. Much of the research on cultural aspects of weight have focused on female populations. For example, Shroff and Thompson (2004) examined weight stigma experiences and body (dis)satisfaction and found that among female undergraduate students in India $(N=93)$, a history of weight-based teasing, or stigma, mediated the relationship between BMI and body dissatisfaction. Similarly, Reddy and Crowther (2007), found that among South East Asian women $(N=74)$ weight-based teasing was significantly associated with body dissatisfaction and body dissatisfaction played a mediating role between weight stigma experiences and eating attitudes and behaviors. These studies suggest that weight stigma experienced by women in other cultures has similar effects on body dissatisfaction as Western women irrespective of cultural context. These findings suggest that weight stigma is associated with negative consequences such as body dissatisfaction and negative attitudes towards eating across cultures and further contributes to disordered eating behaviors and physical activity behaviors.

Another important avenue of weight stigma research includes exploring the impact of weight-based discrimination and stigma on health behaviors such as eating behaviors and physical activity among obese individuals. Emerging research in this area suggests that there may be associations between weight stigma experiences and increased risk of unhealthy eating behaviors and avoidance of physical activity, both of which are crucial components of successful weight management practices. The majority of research investigating the relationship between weight stigma and eating behaviors has looked at experiences of binge eating. Several studies support findings that experiences of weight stigma are associated with more frequent binge 
eating behaviors (e.g., Jackson, Grilo, \& Masheb, 2000), however, some studies suggest that psychological distress may be an important mediator of the relationship (e.g., Ashmore, Friedman, Reichmann, \& Musante, 2008). Thus, it may be a presence of psychological distress as a result of weight stigma experiences that increases individuals' vulnerability to binge eating behaviors. Weight stigma and binge-eating behaviors among nonclinical samples have shown similar results supporting that there is a positive relationship between the number of weight stigma experiences and frequency of binge-eating (Annis et al., 2004). However, some research has failed to find associations between eating behaviors and weight stigma, suggesting that the relationship between weight stigma and eating behaviors is quite complex (Puhl \& Heuer, 2009). In response to mixed findings across research, Womble et al., (2001) found that risk for bingeeating behaviors among young obese adults $(N=808 ; 55 \%$ women $)$ included an interaction between weight-targeted teasing, body dissatisfaction, restrictive eating, frequent weight fluctuations, and negative emotionality. These results suggest that the relationship between weight stigma and unhealthy eating practices involves a complex interaction of many variables including components of weight stigma experiences, body dissatisfaction, and negative emotionality. A growing body of literature indicates that the internalization of weight stigma experiences, beliefs, and stereotypes shows promise for understanding the relationship with maladaptive eating behaviors. For example, Puhl, Moss-Racusin, \& Schwartz (2007) found that among adult women $(N=1,013)$ those who internalized weight-based stereotypes reported more frequent binge-eating episodes compared to women that did not internalize weight stereotypes. With these encouraging findings, the impact of variables such as onset of weight stigma exposure, onset of obesity, mediating effects of impaired mood and psychological distress, 
internalization of weight stigma beliefs and stereotypes, and body dissatisfaction warrant further study.

Regular physical activity is a major focus for the prevention of obesity; however, research shows that experiences of weight stigma may lead overweight and obese individuals to engage in exercise avoidance (Puhl \& Leudicke, 2012). For example, Vartanian and Shaprow (2008) surveyed female college students on self-reported weight stigma, exercise motives, and exercise behaviors and found a correlation between weight stigma experiences and avoidance of exercise engagement, even after controlling for body dissatisfaction. These results suggest that experiences of weight stigma may result in an avoidance of exercise engagement. Although research investigating the relationships between weight stigma, eating behaviors, and physical activity requires increased attention, the existing evidence suggests that experiences of weight stigma may increase the risk of engaging in unhealthy eating practices and avoidance of physical activity and exercise, thus challenging traditional perceptions that weight stigma may motivate individuals towards healthy behaviors.

Weight stigma is a pervasive social issue among overweight and obese populations. Sources of weight stigma for these individuals originate from the social context and typically include comments and messages from close interpersonal relationships and significant others such as family and friends. These weight-based comments have been shown to have negative impacts on self-esteem, foster body dissatisfaction, and increase risk for depression.

Behaviorally, individuals that experience weight stigma in their lives may internalize negative messages about weight and resort to unhealthy eating practices such as binge eating as a means of coping. Additionally, the internalization of anti-fat and weight stigma messages may foster the avoidance of engaging in exercise which, based on physical activity literature, conclusively 
supports exercise engagement for obesity treatment. Finally, these negative effects of weight stigma have been shown to be stable across cultures, and predominately originate from social contexts such as from healthcare professionals and exercise contexts.

\section{Fitness center environments}

Individuals of all weight ranges engage in exercise in the context of fitness centers, with approximately one-third of exercising women engage in exercise in fitness centers (Slater \& Tiggemann, 2006). Within fitness center environments, a variety of exercise equipment is provided for the purpose of different types of fitness and health activities that encourage a healthy lifestyle. Unfortunately, research indicates that not all individuals benefit positively from all types of exercise (Ackard, Brehm, \& Steffen, 2002; Calogero \& Pedrotty, 2007). Many facilities include group fitness classes (cardio-based, weight-based, and yoga-based), and individual workouts (cardio-based and weight-based). Within body image literature in exercise contexts, fitness centers have been identified as environments will an increased focus on bodily appearance, containing many elements which amplifies the body and physical features (Prichard \& Tiggemann, 2005). Some of these elements which objectify the female body include multiple full-length mirrors (e.g., Prichard \& Tiggemann, 2010), posters that idealize the female body, direct comparison with other women, and revealing workout attire (Prichard \& Tiggemann, 2008). Many fitness centers promote the message that one can change their body shape and size through regimented exercise for weight-loss or appearance-motives through objectifying features within the exercise environment.

Objectification of the female body. Correlational research argues that fitness center environments promote the objectification of the female body (e.g., Prichard \& Tiggemann, 2005; Slater \& Tiggemann, 2006), communicating that aesthetic body ideals can be achieved through 
routine physical sculpting. Because fitness center environments consist of elements which magnify appearance and promote aesthetic ideals, women exercise body pressures and develop a desire to meet established cultural ideals (Tiggemann \& Lynch, 2001). Within women 16-25 years, those that are members of fitness centers have been shown to display higher levels of selfobjectification, self-surveillance and disordered eating behaviors than women who are not fitness center members (Strelan et al., 2003). Consistent exposure to body pressures in fitness centers contributes to self-objectification - defined as adopting body ideal expectations as one's own and engaging in habitual monitoring of one's outward appearance (Fredrickson \& Roberts, 1997). Prichard and Tiggemann (2005) uniquely predicted that time spent exercising in a fitness center would be associated with more self-objectification among women 18-45 years. More recent longitudinal research extended Prichard and Tiggemann's (2005) study by examining selfobjectification over a 12-month period among women 16-68 years. Interestingly, their results demonstrated that young adult women 16-28 years showed increased self-objectification over the 12-month period for young participants who remained fitness center members (Prichard \& Tiggemann, 2012). Furthermore, participants in this study had similar initial levels of selfobjectification to non-fitness center members, indicating that women high on self-objectification did not self-select into the fitness center environment. Research supports a link between selfobjectification with motives to exercise which are primarily appearance-based reasons whereas exercising for health and fitness-based motives has been associated with lower selfobjectification (Prichard \& Tiggemann, 2012; Strelan et al., 2003) indicating the importance of understanding young adult women's motivations for exercise.

Motives for exercise in women. Research suggests that a number of factors, including reasons for exercise (i.e., appearance-focused versus health-focused) influences the relationships 
between exercise, body image, and eating concerns (Ackard, Brehm, \& Steffen, 2002). As previously noted, the cognitions that women hold towards exercise, including motives for exercise, may be critical in understanding whether exercise protects, or is detrimental to, body image. Motives to exercise have been categorized into three distinct domains including: physical health-related reasons (e.g., to improve physical fitness and health), mental health-related reasons (e.g., to reduce stress or for enjoyment), and appearance-related reasons (e.g., to lose weight or improve appearance; e.g., Furnham, Badmin, \& Sneade, 2002; Strelan, Mehaffey, \& Tiggemann, 2003). Many women exercise to influence body weight and shape (Furnham et al., 2002), motivated to tone their muscles to enhance body shape by engaging spot toing of specific body parts such as stomach and thighs (Markula, 1995). Exercising with the intention of changing one's appearance has consistently been associated with increased body image dissatisfaction, self-objectification, and greater eating pathology (Adkins \& Keel, 2005; DiBartolo, Lin, Montoya, Neal, \& Shaffer, 2007); Strelan et al., 2003; Tiggemann \& Williamson, 2000). In contrast, women that engage in exercise for health and functional reasons display increased body satisfaction as well as lower levels of body dissatisfaction (Strelan et al., 2003; Tiggemann \& Williamson, 2000). These findings suggest that fitness centers have the potential to encourage health-based motives for exercise among young adult women, however current findings indicate that fitness centers continue to represent environments which encourage appearance-based exercise engagement.

In sum, the promotion of most fitness centers to change one's body through exercising for weight-loss or appearance-motives further promotes self-objectification and encourages a negative self-evaluative process among female exercisers contributing to body image concerns, 
and increased vulnerability to unhealthy weight management practices (e.g., over-exercising, disordered eating) in the pursuit of body ideal expectations (Chang, Pan, \& Shu, 2018).

\section{Summary}

Body image is a multidimensional, psychological experience of embodiment, encompassing body-related self-perceptions and attitudes including thoughts, beliefs, feelings, and behaviors (Cash, 2004). The majority of women are dissatisfied with their bodies (Neighbors $\&$ Sobal, 2007) and resort to dieting to achieve a specific body ideal (Johnson, Edwards, \& Gidycz, 2015). Although negative body image has traditionally been investigated as a psychological deficiency which has targeted perceptual components of body image (see Miyake et al., 2010), evidence suggests that to fully understand and improve body image, these phenomena must be conceptualized as fluid, socioculturally-driven experiences (see Cash, 2012).

Sociocultural and environmental factors that contribute to a negative body image have been increasingly explored within the sport context over the last decade (e.g., Reel, Petrie, SooHoo \& Anderson, 2013). For example, weight pressure, referred to more broadly as "body pressure" in this document, is real or perceived coercion from significant others, societal messaging, and/or the culture of sport to change or maintain one's body weight, shape, size, or

appearance (Reel, SooHoo, Gill, \& Jamieson, 2005). Most body pressure research has focused on collegiate female athletes (e.g., Reel et al., 2013) and those in aesthetic sports (e.g., Voelker \& Reel, 2015). Some of the most cited body pressures within the sport environment include formfitting and revealing athletic attire and appearance expectations communicated by coaches, judges, and teammates (Reel et al., 2013). A preponderance of cross-sectional and preliminary longitudinal evidence suggests internalizing these sport-related body pressures contributes to 
negative body image, negative affect, and eating pathology in female athletes (e.g., Krentz \& Warschburger, 2013; Voelker, Gould, \& Reel, 2014).

However, body pressures originating specifically from fitness environments are less understood. Within weight management populations, body pressures - namely weight stigma - has consistently demonstrated significant negative effects on individuals' body perceptions, psychological health, and physical activity engagement (e.g., see Puhl \& Heuer, 2009). Structural components of exercise environments have been identified as sources of body pressure for overweight and obese populations, supporting the argument that how exercise environments are created can affect how individuals conceptualize their body image. Irrespective of BMI, not all individuals seem to benefit equally from all exercise types, despite evidence suggesting exercise can improve body image (Campbell \& Hausenblas, 2009). Approximately one third of exercising women participate in fitness centers (Slater \& Tiggemann, 2006) - a context associated with body image concerns (Prichard \& Tiggemann, 2005). Young adult women aged 18-25 years who exercise in fitness centers have been identified as particularly at risk for body image concerns for several reasons. Evidence supports that many young adult women begin exercising with the intention of altering body shape and attractiveness, and these appearance-focused motives for exercise have been consistently associated with decreased self-esteem and body dissatisfaction (Furnham, Badmin, \& Sneade, 2002; Tiggemann \& Williamson, 2000). Furthermore, the fitness center environment has been described as a context in which appearance and body shape are magnified, which presents added context-specific body pressures for young adult female exercisers (Prichard \& Tiggemann, 2012). Together, the abundance of literature in body image, weight pressures in sport, and weight stigma in obesity serves as a template by which to examine 
these constructs within young adult women in fitness center environments and supports the application of these well-known constructs to a novel population.

\section{Works Cited}

Ackard, D. M., Brehm, B. J., \& Steffen, J. J. (2002). Exercise and eating disorders in collegeaged women: Profiling excessive exercisers. Eating Disorders, 10(1), 31-47.

Adkins, E. C., \& Keel, P. K. (2005). Does "excessive" or "compulsive" best describe exercise as a symptom of bulimia nervosa?. International Journal of Eating Disorders, 38(1), 24-29.

Ahadzadeh, A. S., Sharif, S. P., \& Ong, F. S. (2017). Self-schema and self-discrepancy mediate the influence of Instagram usage on body image satisfaction among youth. Computers in Human Behavior, 68, 8-16.

American Psychiatric Association. (2013). Diagnostic and statistical manual of mental disorders ( $5^{\text {th }}$ ed.). Washington, DC: American Psychiatric Association.

Anderson, C., \& Petrie, T. A. (2012). Prevalence of disordered eating and pathogenic weight control behaviors among NCAA division I female collegiate gymnasts and swimmers. Research Quarterly for Exercise and Sport, 83(1), 120-124.

Anderson, C. M., Petrie, T. A., \& Neumann, C. S. (2011). Psychosocial correlates of bulimic symptoms among NCAA division-I female collegiate gymnasts and swimmers/divers. Journal of sport and exercise psychology, 33(4), 483-505.

Anderson, C. M., Petrie, T. A., \& Neumann, C. S. (2012). Effects of sport pressures on female collegiate athletes: A preliminary longitudinal investigation. Sport, Exercise, and Performance Psychology, 1(2), 120. 
Annis, N. M., Cash, T. F., \& Hrabosky, J. I. (2004). Body image and psychosocial differences among stable average weight, currently overweight, and formerly overweight women: the role of stigmatizing experiences. Body image, 1(2), 155-167.

Ashmore, J. A., Friedman, K. E., Reichmann, S. K., \& Musante, G. J. (2008). Weight-based stigmatization, psychological distress, \& binge eating behavior among obese treatmentseeking adults. Eating Behaviors, 9(2), 203-209.

Barker, E. T., \& Galambos, N. L. (2003). Body dissatisfaction of adolescent girls and boys: Risk and resource factors. The Journal of Early Adolescence, 23(2), 141-165.

Berscheid, E., Walster, E., \& Bohrnstedt, G. (1973). The happy American body: A survey report. Berry, T., \& Howe, B. (2000). Risk factors for disordered eating in female university athletes. Journal of sport behavior, 23(3), 207.

Boghi, A., Sterpone, S., Sales, S., D'Agata, F., Bradac, G. B., Zullo, G., \& Munno, D. (2011). In vivo evidence of global and focal brain alterations in anorexia nervosa. Psychiatry Research: Neuroimaging, 192(3), 154-159.

Boepple, L., \& Thompson, J. K. (2016). A content analytic comparison of fitspiration and thinspiration websites. International Journal of Eating Disorders, 49(1), 98-101.

Bordo, S. (2004). Unbearable weight: Feminism, Western culture, and the body. University of California Press.

Brewis, A., Sreetharan, C. S., \& Wutch, A. (2018). Obesity stigma as a globalizing health challenge. Globalization and Health, 14:20. doi:10.1186/s12992-018-0337-x

Brochu, P. M., \& Esses, V. M. (2011). What's in a name? The effects of the labels "fat" versus “overweight” on weight bias. Journal of Applied Social Psychology, 41(8), 1981-2008. 
Brown, Z., \& Tiggemann, M. (2016). Attractive celebrity and peer images on Instagram: Effect on women's mood and body image. Body image, 19, 37-43.

Byrne, S., \& McLean, N. (2002). Elite athletes: effects of the pressure to be thin. Journal of Science and Medicine in Sport, 5(2), 80-94.

Cafri, G., Yamamiya, Y., Brannick, M., \& Thompson, J. K. (2005). The influence of sociocultural factors on body image: A meta-analysis. Clinical Psychology: science and practice, 12(4), 421-433.

Calogero, R., \& Pedrotty, K. (2007). Daily practices for mindful exercise. In Low-cost approaches to promote physical and mental health (pp. 141-160). Springer, New York, NY.

Campbell, A., \& Hausenblas, H. A. (2009). Effects of exercise interventions on body image: A meta-analysis. Journal of health psychology, 14(6), 780-793.

Carr, D., \& Friedman, M. A. (2006). Body weight and the quality of interpersonal relationships. Social Psychology Quarterly, 69(2), 127-149.

Carr, D., Friedman, M. A., \& Jaffe, K. (2007). Understanding the relationship between obesity and positive and negative affect: the role of psychosocial mechanisms. Body image, 4(2), $165-177$.

Carrigan, K. W., Petrie, T. A., \& Anderson, C. M. (2015). To weigh or not to weigh? relation to disordered eating attitudes and behaviors among female collegiate athletes. Journal of Sport and Exercise Psychology, 37(6), 659-665.

Cash, T. F. (1994). Body-image attitudes: Evaluation, investment, and affect. Perceptual and Motor skills, 78(3_suppl), 1168-1170.

Cash, T. F. (2004). Body image: past, present, and future. Body Image, 1(1), 1-5. 
Cash, T. F., \& Green, G. K. (1986). Body weight and body image among college women:

Perception, cognition, and affect. Journal of personality assessment, 50(2), 290-301.

Cash, T. F., Phillips K. A., Santos, M. T., \& Hrabosky, J. I. (2004). Measuring 'negative body image': validation of the body image disturbance questionnaire in a nonclinical population. Body Image, 1(4),363-372.

Cash, T. F., Wood, K. C., Phelps, K. D., \& Boyd, K. (1991). New assessments of weight-related body image derived from extant instruments. Perceptual and Motor Skills, 73(1), 235241.

Chang, C. C., Pan, M. C., \& Shu, S. T. (2018). Relationship between self-presentation, exercise dependence, and perceived body image. International Journal of Sport and Exercise Psychology, 1-13. doi:10.1080/1612197X.2018.1444078

Cheek, C. A., Hill, K., Carlson, J., Lock, J., \& Peebles, R. (2015). 171. Weight-related Coaching Pressures, Mental Health, Sleep, and Quality of Life in Competitive University Athletes. Journal of Adolescent Health, 56(2), S88.

Chen, E. Y., Bocchieri-Ricciardi, L. E., Munoz, D., Fischer, S., Katterman, S., Roehrig, M., ... \& Le Grange, D. (2007). Depressed mood in class III obesity predicted by weight-related stigma. Obesity Surgery, 17(5), 669-671.

DiBartolo, P. M., Lin, L., Montoya, S., Neal, H., \& Shaffer, C. (2007). Are there "healthy" and "unhealthy" reasons for exercise? Examining individual differences in exercise motivations using the function of exercise scale. Journal of Clinical Sport Psychology, 1(2), 93-120.

Fardouly, J., \& Vartanian, L. R. (2016). Social media and body image concerns: Current research and future directions. Current opinion in psychology, 9, 1-5. 
Festinger, L. (1954). A theory of social comparison processes. Human relations, 7(2), 117-140.

Francisco, R., Narciso, I., \& Alarcao, M. (2013). Parental influences on elite aesthetic athletes' body image dissatisfaction and disordered eating. Journal of Child and Family Studies, 22(8), 1082-1091.

Fredrickson, B. L., \& Roberts, T. A. (1997). Objectification theory: Toward understanding women's lived experiences and mental health risks. Psychology of women quarterly, 21(2), 173-206.

Friedman, K. E., Reichmann, S. K., Costanzo, P. R., Zelli, A., Ashmore, J. A., \& Musante, G. J. (2005). Weight stigmatization and ideological beliefs: relation to psychological functioning in obese adults. Obesity, 13(5), 907-916.

Furnham, A., Badmin, N., \& Sneade, I. (2002). Body image dissatisfaction: Gender differences in eating attitudes, self-esteem, and reasons for exercise. The Journal of psychology, 136(6), 581-596.

Greenleaf, C. (2002). Athletic body image: Exploratory interviews with former competitive female athlete. Women in Sport and Physical Activity Journal, 11(1), 63-88.

Greenleaf, C. (2004). Weight pressures and social physique anxiety among collegiate synchronized skaters. Journal of Sport Behavior, 27(3), 260.

Greenleaf, C., Petrie, T. A., Carter, J., \& Reel, J. J. (2009). Female collegiate athletes: Prevalence of eating disorders and disordered eating behaviors. Journal of American College Health, 57(5), 489-496.

Grogan, S. (2010). Promoting positive body image in males and females: contemporary issues and future directions. Sex Roles, 63(9-10), 757-765. 
Hausenblas, H. A., \& Carron, A. V. (2002). Assessing eating disorder symptoms in sport groups: A critique with recommendations for future research. International Sports Journal, 6(1), $65-74$.

Hausenblas, H. A., Cook, B. J., \& Chittester, N. I. (2008). Can exercise treat eating disorders?. Exercise and Sport Sciences Reviews, 36(1), 43-47.

Hausenblas, H. A., \& Fallon, E. A. (2006). Exercise and body image: A metaanalysis. Psychology and Health, 21(1), 33-47.

Hendrickse, J., Arpan, L. M., Clayton, R. B., \& Ridgway, J. L. (2017). Instagram and college women's body image: Investigating the roles of appearance-related comparisons and intrasexual competition. Computers in Human Behavior, 74, 92-100.

Hendrickse, J., Secharan, R., \& Clayton, R. B. (2016). Examining women's cognitive and emotional processing of thin, average, and plus size fashion models depicted in the media. Psychophysiology, 53, S48.

Homan, K. J., \& Tylka, T. L. (2014). Appearance-based exercise motivation moderates the relationship between exercise frequency and positive body image. Body Image, 11(2), $101-108$.

Jackson, T. D., Grilo, C. M., \& Masheb, R. M. (2000). Teasing history, onset of obesity, current eating disorder psychopathology, body dissatisfaction, and psychological functioning in binge eating disorder. Obesity, 8(6), 451-458.

Karr, T. M., Davidson, D., Bryant, F. B., Balague, G., \& Bohnert, A. M. (2013). Sport type and interpersonal and intrapersonal predictors of body dissatisfaction in high school female sport participants. Body image, 10(2), 210-219. 
Kerr, G., Berman, E., \& Souza, M. J. D. (2006). Disordered eating in women's gymnastics: Perspectives of athletes, coaches, parents, and judges. Journal of Applied Sport Psychology, 18(1), 28-43.

Kong, P., \& Harris, L. M. (2015). The sporting body: body image and eating disorder symptomatology among female athletes from leanness focused and nonleanness focused sports. The Journal of psychology, 149(2), 141-160.

Krane, V., Stiles-Shipley, J. A., Waldron, J., \& Michalenok, J. (2001). Relationships Among Body Satisfaction, Social Physique Anxiety, and Eating Behaviors in Female Athletes and Exercisers. Journal of sport behavior, 24(3).

Lawler, M., \& Nixon, E. (2011). Body dissatisfaction among adolescent boys and girls: the effects of body mass, peer appearance culture and internalization of appearance ideals. Journal of youth and adolescence, 40(1), 59-71.

Lieberman, M., Gauvin, L., Bukowski, W. M., \& White, D. R. (2001). Interpersonal influence and disordered eating behaviors in adolescent girls: The role of peer modeling, social reinforcement, and body-related teasing. Eating behaviors, 2(3), 215-236.

Markula, P. (1995). Firm but shapely, fit but sexy, strong but thin: The postmodern aerobicizing female bodies. Sociology of sport journal, 12(4), 424-453.

Miyake, Y., Okamoto, Y., Onoda, K., Kurosaki, M., Shirao, N., Okamoto, Y., \& Yamawaki, S. (2010). Brain activation during the perception of distorted body images in eating disorders. Psychiatry Research: Neuroimaging, 181(3), 183-192. doi:10.1016/j.pscychresns.2009.09.001 
Moradi, B. (2010). Addressing gender and cultural diversity in body image: Objectification theory as a framework for integrating theories and grounding research. Sex Roles, 63(12), 138-148.

Moradi, B., Dirks, D., \& Matteson, A. V. (2005). Roles of sexual objectification experiences and internalization of standards of beauty in eating disorder symptomatology: A test and extension of objectification theory. Journal of Counseling Psychology, 52(3), 420.

Murnen, S. K., \& Smolak, L. (2019). The Cash effect: Shaping the research conversation on body image and eating disorders. Body image. doi:10.1016/j.bodyim.2019.01.001

Muscat, A. C., \& Long, B. C. (2008). Critical comments about body shape and weight: Disordered eating of female athletes and sport participants. Journal of Applied Sport Psychology, 20(1), 1-24.

Neumark-Sztainer, D., Paxton, S. J., Hannan, P. J., Haines, J., \& Story, M. (2006). Does body satisfaction matter? Five-year longitudinal associations between body satisfaction and health behaviors in adolescent females and males. Journal of adolescent health, 39(2), 244-251.

Puhl, R., \& Brownell, K. D. (2001). Bias, discrimination, and obesity. Obesity, 9(12), 788-805.

Puhl, R. M., \& Brownell, K. D. (2006). Confronting and coping with weight stigma: an investigation of overweight and obese adults. Obesity, 14(10), 1802-1815.

Puhl, R. M., \& Heuer, C. A. (2009). The stigma of obesity: a review and update. Obesity, 17(5), 941-964.

Puhl, R. M., Moss-Racusin, C. A., \& Schwartz, M. B. (2007). Internalization of weight bias: implications for binge eating and emotional well-being. Obesity, 15(1), 19-23. 
Puhl, R. M., Moss-Racusin, C. A., Schwartz, M. B., \& Brownell, K. D. (2007). Weight stigmatization and bias reduction: perspectives of overweight and obese adults. Health education research, 23(2), 347-358.

Prichard, I., McLachlan, A. C., Lavis, T., \& Tiggemann, M. (2018). The impact of different forms of\# fitspiration imagery on body image, mood, and self-objectification among young women. Sex Roles, 78(11-12), 789-798.

Radtke, T., Kaklamanou, D., Scholz, U., Hornung, R., \& Armitage, C.J. (2014). Are diet-specific compensatory health beliefs predictive of dieting intentions and behaviour? Appetite, 76, $36-43$.

Reddy, S. D., \& Crowther, J. H. (2007). Teasing, acculturation, and cultural conflict: Psychosocial correlates of body image and eating attitudes among South Asian women. Cultural Diversity and Ethnic Minority Psychology, 13(1), 45.

Reel, J. J. (2012). Identification and prevention of weight pressures and body image concerns among athletes. Latin American Journal of Sport Psychology, 6(2), 203-216.

Reel, J. J., \& Gill, D. L. (1998). Weight concerns and disordered eating attitudes among male and female college cheerleaders. Women in Sport and Physical Activity Journal, 7(2), 7994.

Reel, J. J., Greenleaf, C., Baker, W. K., Aragon, S., Bishop, D., Cachaper, C., ... \& Hattie, J. (2007). Relations of body concerns and exercise behavior: A meta-analysis. Psychological reports, 101(3), 927-942.

Reel, J. J., Petrie, T. A., SooHoo, S., \& Anderson, C. M. (2013). Weight pressures in sport: Examining the factor structure and incremental validity of the weight pressures in sportFemales. Eating behaviors, 14(2), 137-144. 
Reel, J. J., Jamieson, K. M., SooHoo, S., \& Gill, D. L. (2005). Femininity to the extreme: Body image concerns among college female dancers. Women in sport and physical activity journal, 14(1), 39-51.

Reel, J. J., SooHoo, S., Petrie, T. A., Greenleaf, C., \& Carter, J. E. (2010). Slimming down for sport: Developing a weight pressures in sport measure for female athletes. Journal of Clinical Sport Psychology, 4(2), 99-111.

Rogge, M. M., Greenwald, M., \& Golden, A. (2004). Obesity, stigma, and civilized oppression. Advances in Nursing Science, 27(4), 301-315.

Rosen, L. W., \& Hough, D. O. (1988). Pathogenic weight-control behaviors of female college gymnasts. The Physician and Sportsmedicine, 16(9), 140-144.

Rosenblum, G. D., \& Lewis, M. (1999). The relations among body image, physical attractiveness, and body mass in adolescence. Child development, 70(1), 50-64.

Rosenberger, P. H., Henderson, K. E., \& Grilo, C. M. (2006). Correlates of body image dissatisfaction in extremely obese female bariatric surgery candidates. Obesity surgery, 16(10), 1331-1336.

Sherman, R. T., \& Thompson, R. A. (2009). Body image and eating disturbance in athletes: Competing to win or to be thin. The hidden faces of eating disorders and body image, 938.

Shroff, H., \& Thompson, J. K. (2004). Body image and eating disturbance in India: Media and interpersonal influences. International Journal of Eating Disorders, 35(2), 198-203.

Stice, E. (1999). Clinical implications of psychosocial research on bulimia nervosa and bingeeating disorder. Journal of Clinical Psychology, 55(6), 675-683. 
Strelan, P., Mehaffey, S. J., \& Tiggemann, M. (2003). Brief report: Self-objectification and esteem in young women: The mediating role of reasons for exercise. Sex Roles, 48(1-2), $89-95$.

Stronge, S., Greaves, L. M., Milojev, P., West-Newman, T., Barlow, F. K., \& Sibley, C. G. (2015). Facebook is linked to body dissatisfaction: Comparing users and non-users. Sex Roles, 73(5-6), 200-213.

Sundgot-Borgen, J. (1994). Risk and trigger factors for the development of eating disorders in female elite athletes. Medicine \& Science in Sports \& Exercise, 26(4), 414-419.

Szymanski, D. M., Moffitt, L. B., \& Carr, E. R. (2011). Sexual objectification of women: Advances to theory and research $1 \psi 7$. The Counseling Psychologist, 39(1), 6-38.

Thompson, R. A., \& Sherman, R. T. (2011). Eating disorders in sport. Routledge.

Tiggemann, M. (2005). Body dissatisfaction and adolescent self-esteem: Prospective findings. Body image, 2(2), 129-135.

Tiggemann, M., \& Lynch, J. E. (2001). Body image across the life span in adult women: The role of self-objectification. Developmental psychology, 37(2), 243.

Tiggemann, M., \& McGill, B. (2004). The role of social comparison in the effect of magazine advertisements on women's mood and body dissatisfaction. Journal of Social and Clinical Psychology, 23(1), 23-44.

Torstveit, M. K., Rosenvinge, J. H., \& Sundgot-Borgen, J. (2008). Prevalence of eating disorders and the predictive power of risk models in female elite athletes: a controlled study. Scandinavian journal of medicine \& science in sports, 18(1), 108-118.

Tylka, T. L., \& Sabik, N. J. (2010). Integrating social comparison theory and self-esteem within objectification theory to predict women's disordered eating. Sex roles, 63(1-2), 18-31. 
Van Strien, T., Frijters, J. E., Van Staveren, W. A., Defares, P. B., \& Deurenberg, P. (1986). The predictive validity of the Dutch restrained eating scale. International Journal of Eating Disorders, 5(4), 747-755.

Varnes, J. R., Stellefson, M. L., Janelle, C. M., Dorman, S. M., Dodd, V., \& Miller, M. D. (2013). A systematic review of studies comparing body image concerns among female college athletes and non-athletes, 1997-2012. Body image, 10(4), 421-432.

Vartanian, L. R., \& Shaprow, J. G. (2008). Effects of weight stigma on exercise motivation and behavior: a preliminary investigation among college-aged females. Journal of health psychology, 13(1), 131-138.

Voelker, D. K., \& Reel, J. J. (2015). An Inductive Thematic Analysis of Female Competitive Figure Skaters' Experiences of Weight Pressure in Sport. Journal of Clinical Sport Psychology, 9(4), 297-316.

Washington, R. L. (2011). Peer reviewed: Childhood obesity: Issues of weight bias. Preventing chronic disease, 8(5).

Womble, L. G., Williamson, D. A., Martin, C. K., Zucker, N. L., Thaw, J. M., Netemeyer, R., ... \& Greenway, F. L. (2001). Psychosocial variables associated with binge eating in obese males and females. International Journal of Eating Disorders, 30(2), 217-221. 